\title{
Circumstellar Dust Disks in Taurus-Auriga: The Submillimeter Perspective
}

\author{
Sean M. Andrews \\ and \\ Jonathan P. Williams \\ Institute for Astronomy, University of Hawaii, 2680 Woodlawn Drive, Honolulu, HI 96822 \\ andrews@ifa.hawaii.edu, jpw@ifa.hawaii.edu
}

\begin{abstract}
We present a sensitive, multiwavelength submillimeter continuum survey of 153 young stellar objects in the Taurus-Auriga star formation region. The submillimeter detection rate is $61 \%$ to a completeness limit of $\sim 10 \mathrm{mJy}(3-\sigma)$ at $850 \mu \mathrm{m}$. The inferred circumstellar disk masses are log-normally distributed with a mean mass of $\sim 5 \times 10^{-3} \mathrm{M}_{\odot}$ and a large dispersion (0.5 dex). Roughly one third of the submillimeter sources have disk masses larger than the minimal nebula from which the solar system formed. The median disk to star mass ratio is $0.5 \%$. The empirical behavior of the submillimeter continuum is best described as $F_{\nu} \propto \nu^{2.0 \pm 0.5}$ between $350 \mu \mathrm{m}$ and $1.3 \mathrm{~mm}$, which we argue is due to the combined effects of the fraction of optically thick emission and a flatter frequency behavior of the opacity compared to the interstellar medium. This latter effect could be due to a substantial population of large dust grains, which presumably would have grown through collisional agglomeration. In this sample, the only stellar property that is correlated with the outer disk is the presence of a companion. We find evidence for significant decreases in submillimeter flux densities, disk masses, and submillimeter continuum slopes along the canonical infrared spectral energy distribution evolution sequence for young stellar objects. The fraction of objects detected in the submillimeter is essentially identical to the fraction with excess nearinfrared emission, suggesting that dust in the inner and outer disk are removed nearly simultaneously.
\end{abstract}

Subject headings: circumstellar matter — planetary systems: protoplanetary disks — solar system: formation — stars: pre-main-sequence

\section{Introduction}

The formation and early evolution of stars are intimately coupled to the properties of their accompanying circumstellar disks of gas and dust. These disks also provide the material reservoirs for the assembly of planetary systems. Angular momentum conservation dictates that a collapsing 
molecular cloud core with some initial rotation will result in both a central protostar and a flattened circumstellar disk (e.g., Terebey, Shu, \& Cassen 1984). Indirect observations indicate that disks are essentially ubiquitous in young star clusters, while optical images in silhouette (O'Dell \& Wen 1994) and millimeter spectral line confirmations of Keplerian rotation (e.g., Simon, Dutrey, \& Guilloteau 2001) provide more direct evidence in specific cases. Comparisons of infrared observations with physical models of young stellar objects (YSOs; here taken to mean a young star and its associated circumstellar material) have led to a sequence of evolutionary stages which occur before the start of the main-sequence (Lada \& Wilking 1984; Adams \& Shu 1986; Adams, Lada, \& Shu 1987). In the Class I stage, an extended circumstellar envelope is rapidly dumping material onto a central protostar and a massive accretion disk. After the supply of envelope material is dissipated, the YSO becomes a Class II object, with a disk that is actively accreting material onto a central, optically visible star. In the final Class III stage, at least the inner part of the circumstellar disk has been evacuated, although the dominant physical mechanism for this process remains in debate (see Hollenbach, Yorke, \& Johnstone 2000). The most interesting possibility, at least from a cosmogonical viewpoint, is that the gas and dust in the disk have agglomerated into larger objects in a developing planetary system.

Observations of the morphology of the broadband spectral energy distribution (SED) and various diagnostics of accretion can be used to trace the evolution of a YSO. Longward of $\sim 1 \mu \mathrm{m}$, the SED of a YSO is composed of a continuum of thermal spectra from the radially distributed circumstellar dust, modified by the radiative transfer properties of the grains. Changes in the SED through the evolutionary sequence are indicative of the loss of circumstellar components in the system; first the envelope and then the disk. The slope of the infrared SED is determined by the radial temperature distribution of the circumstellar dust (e.g., Adams, Lada, \& Shu 1987; Beckwith et al. 1990). Therefore, measurements of infrared colors provide a relatively simple observational constraint on the temperature structure of a disk. However, more detailed physical interpretations of the infrared SED are challenging, due to the strong dependence on the relatively unknown radiative transfer properties of the grains and detailed disk structure (e.g., the inner disk radius or vertical scale height).

In the early evolution stages (Class I and II), material from the inner disk is dragged in magnetospheric funnel flows to the stellar surface, with an accretion shock resulting upon impact (see the review by Najita et al. 2000). This process is responsible for the observed continuum excesses (Calvet \& Gullbring 1998; Johns-Krull \& Valenti 2001; Muzerolle et al. 2003) and the shapes and strengths of emission lines in YSOs (Hartmann, Hewett, \& Calvet 1994; Muzerolle, Hartmann, \& Calvet 1998; Muzerolle, Calvet, \& Hartmann 2001). The most common observational measurement providing a breakdown of objects as accreting or non-accreting is the equivalent width $(W)$ of the $\mathrm{H} \alpha$ emission line. Although a standard division at $W=10 \AA$ was set by historical instrument limitations rather than a physical motivation, this criterion provides an effective discriminant as many properties of weak-line (WTTSs; $W \leq 10 \AA$; non-accreting) and classical (CTTSs; $W>10 \AA$; accreting) T Tauri stars are remarkably different (e.g., Ghez, Neugebauer, \& Matthews 1993; Osterloh 
\& Beckwith 1995; Chiang, Phillips, \& Lonsdale 1996; Stelzer \& Neuhäuser 2001).

Millimeter and submillimeter observations of circumstellar disks can provide unique information. These observations probe the cool, outer parts of the disk, where giant planets are expected to form and contamination from the stellar photosphere is negligible. The low submillimeter opacities in disks can be used to extrapolate the surface density of the outer disk into the inner, optically thick regions and therefore determine the total disk mass (Beckwith et al. 1990). Assuming the submillimeter emission arises in an optically thin, isothermal portion of the disk, the flux density $\left(F_{\nu}\right)$ and disk mass $\left(M_{d}\right)$ are directly proportional (Hildebrand 1983):

$$
M_{d}=\frac{d^{2} F_{\nu}}{\kappa_{\nu} B_{\nu}\left(T_{c}\right)}
$$

where $d$ is the distance, $\kappa_{\nu}$ is the opacity, and $B_{\nu}\left(T_{c}\right)$ is the Planck function at a characteristic temperature $T_{c}$. Moreover, observations and theoretical models of the opacity in the submillimeter indicate that $\kappa_{\nu}$ is well-matched by a simple power-law in frequency with index $\beta$, although the proposed normalizations vary significantly (Hildebrand 1983; Wright 1987; Pollack et al. 1994; Henning \& Stognienko 1996). With the same optically thin, isothermal disk assumptions, the submillimeter continuum emission should behave roughly as $F_{\nu} \propto \nu^{2+\beta}$. So, with major caveats (see $\S 3$ and the Appendix), a single submillimeter flux density can give the mass of a disk and $\geq 2$ flux points can reveal the frequency dependence of the opacity. Assuming a uniform grain composition and shape, the frequency behavior of the opacity is set by the size distribution of the grains in the disk. A number of single-dish surveys with single-element (or small arrays of) bolometers have been conducted in the Taurus-Auriga star-forming region to address these issues, most of which were carried out at $1.3 \mathrm{~mm}$ (Weintraub, Sandell, \& Duncan 1989; Beckwith et al. 1990; Adams, Emerson, \& Fuller 1990; Beckwith \& Sargent 1991; Mannings \& Emerson 1994; Osterloh \& Beckwith 1995; Motte \& André 2001). Current instrumentation provides the opportunity for significantly more sensitive observations of disks in the submillimeter.

High resolution observations with (sub-)millimeter interferometers have confirmed that circumstellar dust disks are geometrically thin with radii on the order of 100 AU (e.g., Dutrey et al. 1996; Kitamura et al. 2002). Detailed studies of individual disks reveal molecular gas in Keplerian rotation around the central star (e.g., Weintraub, Masson, \& Zuckerman 1989; Koerner, Sargent, \& Beckwith 1993a,b; Dutrey, Guilloteau, \& Simon 1994; Koerner \& Sargent 1995; Mannings \& Sargent 1997; Duvert et al. 1998; Guilloteau \& Dutrey 1998; Simon, Dutrey, \& Guilloteau 2001; Corder, Eisner, \& Sargent 2005). While molecular gas is the primary reservoir of mass in a disk, it is difficult to directly determine $M_{d}$ from the high resolution spectral line data because the brightest, easily detectable lines (i.e., the rotational transitions of CO) are optically thick (Beckwith \& Sargent 1993; Dutrey et al. 1996) and likely to be severely depleted (Dutrey, Guilloteau, \& Simon 1994, 2003). Interpretation of these lines and those from trace molecular species require sophisticated models of the disk structure (e.g., Dartois, Dutrey, \& Guilloteau 2003; Kamp \& Dullemond 2004) and chemistry (e.g., van Zadelhoff et al. 2001, 2003; Aikawa et al. 2002; Qi et al. 2003). Despite the tremendous amount of information provided by these observations, our knowledge is 
still limited to a relatively few disks on account of the large amount of time which must be invested in an interferometric observation.

Multiwavelength submillimeter data could prove useful in placing observational constraints on the dominant mechanism of planet formation. By comparing with infrared SEDs and diagnostics of accretion, we can investigate the dissipation of disks as a function of radius and see if there is consistency with the timescales expected from the collisional growth of planetesimals. The functional form of the opacity may provide information on the mean grain size distribution in the disk, and therefore evidence for the growth of grains demanded by the standard models of planet formation (e.g., Beckwith, Henning, \& Nakagawa 2000).

In this paper, we present a large catalog of such data for most of the known YSOs in the Taurus-Auriga star-forming region. The survey is uniform, sensitive, and provides the most multiwavelength measurements of the submillimeter continuum spectra of YSOs to date. In $\S 2$ we discuss the observations and data reduction procedures. In $\S 3$ we present a simple disk model and use it to derive circumstellar disk masses, place some new observational constraints on the submillimeter opacity properties of disks, and examine relationships between the disk properties and those of the central stars. The results are discussed in $\S 4$, and our conclusions are summarized in $\S 5$. A brief Appendix is included with a more in-depth discussion of the disk models we employ and comments on some particularly interesting sources.

\section{Observations and Data Reduction}

Simultaneous 450 and $850 \mu \mathrm{m}$ continuum photometry observations of 90 YSOs in the TaurusAuriga star-forming region were obtained with the Submillimeter Common User Bolometer Array (SCUBA: Holland et al. 1999) at the $15 \mathrm{~m}$ James Clerk Maxwell Telescope (JCMT) between 2004 February and 2005 January. Accurate reference coordinates (to $\sim 1^{\prime \prime}$ ) for each object were obtained from the 2MASS Point Source Catalog. The effective FWHM beam diameters for SCUBA photometry are $9^{\prime \prime}$ and $15^{\prime \prime}$ at $450\left(\lambda_{\text {eff }}=443 \mu \mathrm{m}\right)$ and $850 \mu \mathrm{m}\left(\lambda_{\text {eff }}=863 \mu \mathrm{m}\right)$, respectively. The precipitable water vapor (PWV) levels in these observations were $1.6 \mathrm{~mm}$ in the mean, corresponding to zenith opacities of 0.32 at $850 \mu \mathrm{m}$ and 1.73 at $450 \mu \mathrm{m}$. More than $50 \%$ of the observations were conducted in very dry conditions $(\mathrm{PWV} \leq 1.5 \mathrm{~mm})$. The data were acquired in sets of $18 \mathrm{~s}$ integrations in a small nine-point jiggle pattern with the secondary mirror chopping (typically) $60^{\prime \prime}$ in azimuth at $7.8 \mathrm{~Hz}$. Each set consisted of between 15 and 40 integrations, and each source was usually observed for two sets. Frequent skydip observations were used to determine atmospheric extinction as a function of elevation and time. Pointing updates on nearby bright standard sources were conducted between sets of integrations: the rms pointing offsets were $\leq 2^{\prime \prime}$. Mars and Uranus

were used as primary flux calibrators, observed at least once per night when available. The secondary calibrators HL Tau, CRL 618, and CRL 2688 were also observed approximately once every 60 to 90 minutes. 
The demodulated SCUBA data were flatfielded, despiked, and corrected for extinction and residual sky emission using standard tasks in the SURF software package (Jenness \& Lightfoot 1998; Jenness, Lightfoot, \& Holland 1998). The "unused" bolometers in the SCUBA arrays provide a distinct advantage in sky subtraction over the standard simple demodulation utilized for single-element (or small array) detectors. With SCUBA, this technique has resulted in a factor of $\sim 3$ increase in the signal-to-noise ratio (Holland et al. 1999) and should give more robust flux measurements. The mean and standard deviation voltages were used to determine the flux density and rms noise level for each source, after appropriate scaling based on the gain values derived from observations of the calibrators. Repeated observations of the flux calibrators in a given night of observing indicate a systematic uncertainty in these gain factors of $\sim 10 \%$ at $850 \mu \mathrm{m}$ and $\sim 25 \%$ at $450 \mu \mathrm{m}$. These systematic errors dominate the uncertainties for brighter sources. Observations of an additional 44 sources were obtained from the SCUBA online archive and reduced in the same manner, accounting for the differences in filter sets for data taken before 1999 November. In a few cases, observations of the same source from several different nights were combined after the processing to yield very sensitive data. For those cases, the combined data are consistent with the individual datasets when the increased integration time is considered.

The Submillimeter High Angular Resolution Camera (SHARC-II: Dowell et al. 2003) on the $10 \mathrm{~m}$ Caltech Submillimeter Observatory (CSO) telescope was also used to image 39 YSOs in the $350 \mu \mathrm{m}$ continuum between 2004 March and 2005 January. The FWHM beam diameter of the SHARC-II point-spread function at $350 \mu \mathrm{m}$ is roughly $9^{\prime \prime}$, achieved by employing an active dish surface optimization system at the CSO. Due to the low atmospheric transmission at this wavelength, these observations were only conducted when the PWV level was $\leq 1.6 \mathrm{~mm}$, corresponding to $350 \mu \mathrm{m}$ zenith opacities of less than $\sim 2$. Opacity measurements at $225 \mathrm{GHz}$ were taken every 10 minutes with a dedicated tilting water vapor monitor observing at fixed azimuth. The observations were conducted by constantly sweeping the telescope in the vicinity of the source in an alt-az Lissajous pattern, providing small Nyquist-sampled maps. At least three separate maps were taken for each source, with between 120 and 600 s of integration per map. The aforementioned SCUBA calibrators were also observed every 60 to 90 minutes for pointing updates and flux calibration. The SHARC-II data reduction was conducted using the CRUSH software package (Kovács et al. 2005). Flux densities were measured in a circular aperture with a radius of $30^{\prime \prime}$, and rms noise levels were determined from the background pixels. Repeated measurements of standard calibration sources show that the absolute flux calibration is accurate to within $25 \%$.

Our sample was selected primarily from the compilation of Kenyon \& Hartmann (1995), and was designed to contain roughly equal numbers of Class II and III objects, WTTSs and CTTSs, and single and multiple stars. The histograms in Figure 1 summarize some of the key properties of the sample. Table 1 gives a collection of submillimeter properties for 153 YSOs in Taurus-Auriga: 90 sources with new SCUBA and SHARC-II data, 44 with archival SCUBA observations and SHARCII data, 4 with data from the literature, and 15 others with SHARC-II data and flux densities from the literature (see the table notes). This table lists the 350, 450, $850 \mu \mathrm{m}$, and $1.3 \mathrm{~mm}$ flux densities 
(the latter from the literature) and statistical errors (1- $\sigma$ rms noise levels) or 3- $\sigma$ upper limits in units of mJy per beam, disk masses (see $\S 3.2$ ) and submillimeter continuum slopes (see $\S 3.3$ ), and various other relevant properties. The projected FWHM beam diameters at the assumed distance of Taurus-Auriga ( $d=140 \mathrm{pc}$; Elias 1978) are $1260 \mathrm{AU}$ for both 350 and $450 \mu \mathrm{m}$ and $2100 \mathrm{AU}$ for $850 \mu \mathrm{m}$. In this paper, we assume that all of the sources are unresolved, and therefore the values in Table 1 are actually the integrated continuum flux densities (in units of mJy). This assumption is valid for Class II and III sources, where the submillimeter emission originates in a disk with a radius of a few hundred AU at most (see the interferometric observations of Dutrey et al. 1996; Kitamura et al. 2002).

On the other hand, submillimeter continuum maps of the Class I sources in this sample usually show a significant amount of extended emission from the outer envelope in addition to a bright, central concentration of emission (itself perhaps marginally resolved) from the disk and inner envelope (e.g., Chandler \& Richer 2000; Hogerheijde \& Sandell 2000; Shirley et al. 2000; Motte \& André 2001; Chini et al. 2001; Young et al. 2003). The non-mapping photometry observations at 450 and $850 \mu \mathrm{m}$ presented here exclude the extended emission component, and therefore only sample the bright peak of emission which presumably originates from warm dust in the inner envelope and/or a disk. Because of the unknown density structure of the inner envelope, it is not possible to unambiguously determine what fraction of this emission peak is contributed by a compact object (i.e., disk) without interferometric observations (see the discussion by Young et al. 2003, and references therein). For some of these objects, there is also the possibility that the $60^{\prime \prime}$ chop throw would place the "off" position in the extended envelope emission, and therefore the flux densities listed in Table 1 could be slightly underestimated. The reader should keep in mind that the submillimeter properties of Class I YSOs in this paper most likely refer to a combination of disk and envelope contributions. The notes in Table 1 provide references to submillimeter maps of the Class I YSOs in the literature when available.

The primary observational goal of this survey was to take advantage of the stability and efficiency of the SCUBA instrument to obtain a $850 \mu \mathrm{m}$ sample with a relatively uniform flux density limit of $\sim 10 \mathrm{mJy}(3-\sigma)$. The mean $3-\sigma$ upper limit for undetected sources at $850 \mu \mathrm{m}$ in this survey is $8.4 \mathrm{mJy}$ (the median is the same), with a standard deviation in the upper limits of $3.1 \mathrm{mJy}$. For comparison, the same sources in the combined $1.3 \mathrm{~mm}$ surveys in Taurus-Auriga conducted by Beckwith et al. (1990) and Osterloh \& Beckwith (1995) have a mean 3- $\sigma$ upper limit of $19 \mathrm{mJy}$ (median of $16 \mathrm{mJy}$ ) and a standard deviation in the upper limits of $10 \mathrm{mJy}$. If we assume that the submillimeter continuum emission behaves as $F_{\nu} \propto \nu^{2}$ (see $\S 3.3$ ), then a factor of 2.3 can be used to scale the $1.3 \mathrm{~mm}$ measurements with those at $850 \mu \mathrm{m}$. The resulting scaled $1.3 \mathrm{~mm}$ mean upper limit is then $44 \mathrm{mJy}$ (median of $37 \mathrm{mJy}$ ). The distributions of the upper limits of undetected sources are shown in Figure 2. In terms of flux density limits on undetected sources, our survey is roughly a factor of 5 more sensitive than previous single-dish work and is also considerably more uniform. The distributions of the signal-to-noise ratios for detected sources in the various surveys are similar, although there are generally higher ratios at $850 \mu \mathrm{m}$. For the sources common to the 
$850 \mu \mathrm{m}$ and $1.3 \mathrm{~mm}$ samples, the detection rates are $64 \pm 7 \%$ and $47 \pm 6 \%$, respectively.

\section{Results}

\subsection{A Simple Disk Model}

A model of the submillimeter continuum emission is needed to extract physical information (e.g., disk masses) from the data. In order to incorporate some non-negligible optical depth and a radial temperature distribution, the simplistic methods outlined in $\S 2$ (e.g., Equation 1) are passed over in favor of one that fits the disk SED with a power-law structural model (see Adams, Lada, \& Shu 1987; Beckwith et al. 1990). In this scheme, the SED (from the mid-infrared through the submillimeter) is assumed to be generated from thermal reprocessing of starlight by a geometrically thin dust disk, with the flux density given by

$$
F_{\nu}=\frac{\cos i}{d^{2}} \int_{r_{\circ}}^{R_{d}} B_{\nu}\left(T_{r}\right)\left(1-e^{-\tau_{\nu, r} \sec i}\right) 2 \pi r d r
$$

where $i$ is the inclination angle, $r_{\circ}$ the inner radius, $R_{d}$ the outer radius, $B_{\nu}\left(T_{r}\right)$ the Planck function at a radius-dependent temperature, and $\tau_{\nu, r}$ the optical depth of the disk material. ${ }^{1}$ In essence, the flux density is computed by summing the thermal emission from a continuous set of dust annuli weighted by the radiative transfer properties of the material. The radial temperature distribution is taken to be a power law

$$
T_{r}=T_{1}\left(\frac{r}{1 \mathrm{AU}}\right)^{-q}
$$

where $T_{1}$ is the temperature at $r=1 \mathrm{AU}$. The optical depth is the product of the disk opacity, $\kappa_{\nu}$, and the radial surface density profile, $\Sigma_{r}$, which is also taken to be a power law:

$$
\Sigma_{r}=\Sigma_{\circ}\left(\frac{r}{r_{\circ}}\right)^{-p}
$$

We assume that the opacity is a power law in frequency with index $\beta$ and a normalization of $0.1 \mathrm{~cm}^{2}$ $\mathrm{g}^{-1}$ at $1000 \mathrm{GHz}$ (Beckwith et al. 1990). This value assumes a 100:1 mass ratio between gas and dust.

Because a given disk typically has relatively few SED datapoints, fitting the SED with the model described above requires that some of the remaining 8 parameters $\left(i, r_{\circ}, R_{d}, \Sigma_{\circ}, p, T_{1}\right.$, $q, \beta)$ be fixed. Fortunately, the precise values of $i, r_{\circ}$, and $R_{d}$ do not significantly affect the determination of interesting physical parameters as long as they lie in a realistic range. A fiducial

\footnotetext{
${ }^{1}$ Because Equation 2 implicitly assumes a constant source function in the disk, it is only a valid approximation when the inclination angle is not too large. A more sophisticated treatment of radiative transfer is required for nearly edge-on disks (e.g., Chiang \& Goldreich 1999).
} 
set of fixed parameters is adopted here: $i=0^{\circ}, r_{\circ}=0.01 \mathrm{AU}, R_{d}=100 \mathrm{AU}$, and $p=1.5 .^{2}$ The inner and outer disk radii are typical values based roughly on the dust sublimation temperature (e.g., Dullemond, Dominik, \& Natta 2001; Muzerolle et al. 2003) and direct disk size measurements (e.g., Dutrey et al. 1996; Kitamura et al. 2002; Akeson et al. 2005). The surface density index, $p$, is the most difficult parameter to constrain observationally. The value selected here is obtained when the compositions of the planets in the solar system are augmented to cosmic abundances and smeared out into annuli: the Minimum Mass Solar Nebula (MMSN; Weidenschilling 1977). The inclination value is set merely as a computational convenience. The remaining parameters $\left(\Sigma_{\circ}, T_{1}\right.$, $q, \beta)$ must be determined from the data.

\subsection{Disk Masses}

Submillimeter continuum observations provide measurements of disk masses. However, the simplistic conversion of a flux density into a mass via Equation 1 masks some important complications. For example, $M_{d}$ could be uncertain to a factor of $\sim 2$ due to its roughly linear relationship with $T_{c}$. More fundamentally, the relationship between $F_{\nu}$ and $M_{d}$ is nonlinear due to the significant fraction of the submillimeter emission which is optically thick (e.g., Beckwith et al. 1990). By assuming optically thin emission and using Equation 1, $M_{d}$ could be underestimated (particularly for objects with larger flux densities). To avoid these problems and fit the SEDs with the model described by Equation 2, mid- and far-infrared flux densities were taken from the IRAS Point Source Catalog and the compilations of Weaver \& Jones (1992) and Kenyon \& Hartmann (1995). Using data at shorter wavelengths $(\lambda \lesssim 5 \mu \mathrm{m})$ runs the risk of contamination from an extincted photosphere, and therefore introduces more parameters into the problem (e.g., effective temperature, extinction, stellar radius). The submillimeter data presented here were supplemented whenever possible with flux densities from the literature (Adams, Emerson, \& Fuller 1990; Beckwith et al. 1990; Beckwith \& Sargent 1991; Mannings \& Emerson 1994; Osterloh \& Beckwith 1995; Motte \& André 2001). We adopted absolute flux calibration uncertainties of $20 \%$ in the infrared and $25 \%$ $(\lambda \leq 800 \mu \mathrm{m})$ or $20 \%(\lambda>800 \mu \mathrm{m})$ in the submillimeter. Systematic and statistical errors were combined for each individual flux density measurement.

The disk mass and opacity index, $\beta$, are strongly coupled parameters, making it difficult to independently infer their values (see Beckwith \& Sargent 1991). Observations and models of interstellar grains in the molecular ISM, where the material is still diffuse enough to safely assume optically thin thermal emission, indicate that $\beta \approx 2$ (Erickson et al. 1981; Schwartz 1982; Draine $\&$ Lee 1984). However, different mineralogies or grain size distributions in a disk could decrease the index down to $\beta \sim 0$ (e.g., Pollack et al. 1994). Because of the uncertainties in independently measuring $\beta$ and $M_{d}$, we modeled individual SEDs for various values of $\beta$ (between 0 and 2), as well as the typical compromise value for disks, $\beta=1$. Values of $T_{1}, q$, and $\Sigma_{\circ}$ (note that for this

\footnotetext{
${ }^{2}$ See the Appendix for a more detailed examination of the effects of various parameter choices.
} 
model $M_{d} / \mathrm{M}_{\odot} \approx 2 \times 10^{-35} \Sigma_{\circ} / \mathrm{g} \mathrm{cm}^{-2}$ ) for 44 objects in the sample were determined by fitting the SEDs to Equation 2 and minimizing the $\chi^{2}$ statistic. Table 2 gives the results of these fits for $\beta=1$, including the reduced $\chi^{2}$ values $\left(\tilde{\chi}_{\nu}^{2}\right)$, degrees of freedom in the fit $(\nu)$, and references for the infrared and submillimeter SED data from the literature. Much more sophisticated disk models (Men'shchikov, Henning, \& Fischer 1999; Chiang et al. 2001; Semenov et al. 2005) predict disk masses for a few of the same sources which are within a factor of 2-3 of those presented here. Figure 3 shows the distributions of the best-fit values of $q$ and $T_{1}$. The mean values of $q$ and $T_{1}$ are $0.56 \pm 0.08$ and $178 \pm 85 \mathrm{~K}$, respectively (quoted errors are standard deviations of the distributions). We define the "median disk model" to have the above set of fiducial parameters and the median values $q=0.58$ and $T_{1}=148 \mathrm{~K}$, as well as $\beta=1$. Due to the high optical depths at infrared wavelengths compared to the submillimeter, the parameters of the temperature profile, $T_{1}$ and $q$, are often not strongly affected by changes in $\beta$.

The SEDs of most of the YSOs in the survey sample were not fitted as described above because they either lack data (i.e., there were too few degrees of freedom), are undetected in the submillimeter, or have SEDs which indicate such a simple model is insufficient. However, the results of the SED fitting can be used to determine an empirical conversion between a submillimeter flux density and a disk mass. In Figure 4 we show the relationship between the $850 \mu \mathrm{m}$ flux densities and the best-fit values of $M_{d}$ (for $\beta=1$ ) from the SED fitting. This relationship is well described by a simple power law,

$$
\frac{M_{d}}{\mathrm{M}_{\odot}}=(5 \pm 2) \times 10^{-5}\left[\frac{F_{\nu}(850 \mu \mathrm{m})}{\mathrm{mJy}}\right]^{0.96 \pm 0.03}
$$

which is shown as a solid line in Figure 4. A fit of the same data to Equation 1 (assuming the same opacity function given above, where $\kappa_{\nu}=0.035 \mathrm{~cm}^{2} \mathrm{~g}^{-1}$ at $850 \mu \mathrm{m}$ ) is shown as a dashed line, and gives a best-fit characteristic temperature $T_{c}=20 \mathrm{~K}$. For the median disk model, this value of $T_{c}$ occurs at a disk radius of approximately $30 \mathrm{AU}$. Also shown are the relationships between $F_{\nu}$ and $M_{d}$ for the mean and median disk models. Disk mass values and upper limits for the objects which were not fitted with these models were computed from Equation 5. For sources without $850 \mu \mathrm{m}$ measurements which could not be fitted with a disk model, a similar analysis as above was used to derive values of $M_{d}$ from the $1.3 \mathrm{~mm}$ flux density: $M_{d} / \mathrm{M}_{\odot} \approx 10^{-6}\left(F_{\nu} / \mathrm{mJy}\right)^{1.5}$. Disk masses (or 3- $\sigma$ upper limits) are included in Table 1 for all of the sources in the survey sample. Those masses, which use $\beta=1$, will be adopted throughout this paper, unless specifically mentioned otherwise. Our fitting results show that the systematic errors in the disk mass due to the a priori unknown value of $\beta$ are \pm 0.5 dex on average, or a factor of 3 , for a reasonable range of $\beta$ (0 to 2). The $M_{d}$ values inferred for Class I objects should be considered only as upper limits on the disk mass, as there is likely a flux contribution from the inner envelope.

Figure 5 shows the cumulative distributions of the $850 \mu \mathrm{m}$ flux densities and disk masses. This figure shows the distributions of the full sample and a subsample consisting of only those sources which have $\mathrm{a} \geq 3-\sigma$ detection at a submillimeter wavelength. The ordinates in these plots are defined as the probability of an object having a value equal to or greater than the abscissae. 
In both figures, the Kaplan-Meier product limit estimator is used to construct the cumulative distributions for the full sample. ${ }^{3}$ This method allows the incorporation of the $3-\sigma$ upper limits of the $F_{\nu}$ and $M_{d}$ values in the full sample. The computations of probabilities and their errors were conducted with the ASURV Rev. 1.2 software package (LaValley, Isobe, \& Feigelson 1990), following the formalism introduced by Feigelson \& Nelson (1985). A significant caveat with these cumulative distributions is that there is no means to account for the uncertainties in the values of $F_{\nu}$ and $M_{d}$.

Based on the detections subsample distribution in Figure 5 (not incorporating upper limits), we estimate the completeness limit of the survey to be roughly $10 \mathrm{mJy}$. A log-normal distribution of $F_{\nu}$ with mean $1.20 \pm 0.02(16 \mathrm{mJy})$ and variance $1.08 \pm 0.06$ dex provides a good fit to the data for the full sample, whereas a mean of $1.93 \pm 0.01(85 \mathrm{mJy})$ and a variance of $0.41 \pm 0.02$ dex are appropriate for the detections subsample. The distribution of the detections subsample in Figure 5 indicates that $37 \%$ of the YSOs have $M_{d} \geq 0.01 \mathrm{M}_{\odot}$, roughly the total mass of the MMSN (Weidenschilling 1977). Approximately $79 \%$ of the same subsample have disks with masses greater than that of Jupiter. As would be expected from the relationship between $F_{\nu}$ and $M_{d}$ discussed above, the disk masses are also log-normally distributed: the full sample with mean $-3.00 \pm 0.02$ $\left(10^{-3} \mathrm{M}_{\odot}\right)$ and variance $1.31 \pm 0.06 \mathrm{dex}$, and the detections subsample with mean $-2.31 \pm 0.01$ $\left(5 \times 10^{-3} \mathrm{M}_{\odot}\right)$ and variance $0.50 \pm 0.02$ dex.

\subsection{Submillimeter Continuum Slopes}

The slope of the submillimeter continuum emission from a circumstellar disk is empirically well-described by a simple power law in frequency: $F_{\nu} \propto \nu^{\alpha}$. If the emission is assumed to be optically thin and roughly isothermal, Equation 2 can be written $F_{\nu} \propto B_{\nu}(T) \tau_{\nu} \propto \nu^{2+\beta}$ (in the Rayleigh-Jeans limit). However, the submillimeter continuum has a significant contribution from optically thick emission originating in the dense, inner disk which causes a substantial deviation from the $\alpha=2+\beta$ relationship inferred for the optically thin case (Beckwith et al. 1990; Beckwith \& Sargent 1991). We have combined the data presented here with additional flux densities from the literature (Adams, Emerson, \& Fuller 1990; Beckwith et al. 1990; Beckwith \& Sargent 1991; Mannings \& Emerson 1994; Osterloh \& Beckwith 1995; Motte \& André 2001) to determine the values of $\alpha$ given in Table 1 . For objects with more than 2 submillimeter flux densities, $\alpha$ was measured from a linear fit in the $\log \nu$ - $\log F_{\nu}$ plane. When only 1 or 2 flux densities were available, values or 3- $\sigma$ upper limits of $\alpha$ were determined from a simple spectral index.

\footnotetext{
${ }^{3}$ Application of the Kaplan-Meier estimator to the flux density data may be inappropriate. Because each object was observed either until it was detected or a rather uniform flux density limit was reached, the function which describes the censoring of these data is not random. Nevertheless, any effects of using the Kaplan-Meier estimator should only be noticed for flux densities below the completeness limit $(\sim 10 \mathrm{mJy})$. Because multiwavelength SED data were used in determining $M_{d}$ (and various SED morphologies can result in identical values of $M_{d}$ ), the censoring function in that case should be randomized, and therefore the use of the Kaplan-Meier estimator is valid.
} 
Figure 6 compares the submillimeter continuum slopes from 450 to $850 \mu \mathrm{m}$ and $850 \mu \mathrm{m}$ to $1.3 \mathrm{~mm}$. For the full sample, the best-fit slopes are $\alpha=2.06 \pm 0.02$ and $1.93 \pm 0.01$ for the two wavelength regions, respectively. Because the submillimeter continuum emission is generated in the outer disk where temperatures are low, the Rayleigh-Jeans limit criterion is not satisfied (because $h \nu \sim k T$ ) and the continuum slope at shorter wavelengths (nearer to the peak of the thermal emission) could be systematically smaller than at longer wavelengths. However, such an effect is not seen in Figure 6: in fact, the shorter wavelength slope is slightly steeper than at longer wavelengths. This implies that the shape of the submillimeter continuum is not set by the grain temperature distribution alone, but also by the amount of optically thick emission and/or the spectral behavior of the opacity function. Within the uncertainties, it does not significantly matter where in the submillimeter continuum the slope is measured (at least between $350 \mu \mathrm{m}$ and $1.3 \mathrm{~mm}$ ). The slightly shallower best-fit slope for the longer wavelength data may simply be noise, or could be caused by a small fraction of the $1.3 \mathrm{~mm}$ data which are contaminated by non-disk emission: e.g., free-free or gyrosynchrotron radiation from a wind or outflow (e.g., Chiang, Phillips, \& Lonsdale 1996). Another possibility is a real concavity to the long-wavelength SEDs: the models of Pollack et al. (1994) predict a steeper opacity function shortward of $\sim 650 \mu \mathrm{m}$.

The cumulative distribution of $\alpha$ is shown in Figure 7, constructed using the Kaplan-Meier estimator to incorporate $3-\sigma$ upper limits. The median value of $\alpha$ is 2.0 , while only $6 \%$ of the sample has $\alpha \geq 3$, a typical value adopted in the literature due to the (incorrect) assumption of optically thin emission in the Rayleigh-Jeans limit with $\beta=1$. A normal distribution of $\alpha$ with a mean of $1.97 \pm 0.01$ and a variance of $0.22 \pm 0.02$ provides a decent fit to the data, but there is a slightly enhanced probability of larger $\alpha$. Because some of the emission is optically thick, there is no straight-forward means of associating these values of the continuum slope with power law indices of the opacity function $(\beta)$. One approach is to allow $\beta$ to vary in the disk SED models and fit it as an additional parameter (e.g., Beckwith \& Sargent 1991; Mannings \& Emerson 1994; Dutrey et al. 1996). However, in many cases this severely limits the number of degrees of freedom in the fits (see Table 2), which already make a number of assumptions. Beckwith \& Sargent (1991) provide a means of relating $\alpha$ and $\beta$ analytically from other parameters in the SED model fits which essentially indicate that $\beta \propto \alpha$, although there is a constant offset (see the Appendix).

Figure 8 shows the measured values of $\alpha$ as a function of $\log M_{d}$. The shaded region on this diagram marks the functional form of $\alpha\left(M_{d}\right)$ for $\beta=2$ which is representative of the complete range in the measured radial temperature distributions (see Figure 3). The disk mass values are those for $\beta=1$, and the error bar shown to the lower left demonstrates the systematic uncertainty introduced by varying $\beta$ between 0 and 2 . All else being equal, the disk mass is roughly inversely proportional to the opacity, and since larger values of $\beta$ give lower opacities for a fixed frequency, a larger $\beta$ will also give a larger $M_{d}$ (assuming the normalization of the opacity function is fixed). Even allowing for such uncertainties in the disk mass and the range of temperature profiles, Figure 8 indicates that many of the disks in the sample have $\beta<2$ (the curves showing the relationship between $\alpha$ and $M_{d}$ for lower values of $\beta$ always fall in or below the shaded strip: see the Appendix). This 
suggests that the large optical depths in the disk do not completely explain the shallow measured continuum slopes, but that there is also an evolutionary change in the typical opacity properties of dust grains from the ISM (where $\beta=2$ ) to a disk. This result has also been noted in other studies (Beckwith \& Sargent 1991; Mannings \& Emerson 1994; Dutrey et al. 1996; Dent, Matthews, \& Ward-Thompson 1998) for various different sizes and types of samples. It is tempting to conclude that the apparently diminished values of $\beta$ in these disks are due to the collisional growth of dust grains, a necessary condition in any planet formation model (Mizuno 1980; Pollack et al. 1996). Models of the process indicate that grain growth can decrease $\beta$ to values as low as zero (e.g., Miyake \& Nakagawa 1993; Henning, Michel, \& Stognienko 1995). The data in Figure 8 also clearly show that there is no correlation between the disk mass and submillimeter continuum slope.

The coupling of $M_{d}, \beta$, and the opacity normalization makes it difficult to definitively associate low values of the submillimeter continuum slope with decreased opacity indices (Beckwith \& Sargent 1991). The actual value of $\kappa_{\nu}$ is the main uncertainty in the conversion of a submillimeter flux density into a disk mass. Aside from the effect of the grain size distribution (thus the interest in grain growth), both the normalization and $\beta$ depend strongly on the mineralogical composition of the grains (Pollack et al. 1994; Henning \& Stognienko 1996) and their physical shapes (e.g., spherical, fractal, etc.; see Wright 1987). Further discussion of these uncertainties is given by Beckwith et al. (1990) and Beckwith, Henning, \& Nakagawa (2000). As an example, Wright (1987) indicates that $\kappa_{\nu}$ can be roughly an order of magnitude higher for fractal grains compared to spheres at a wavelength of $1 \mathrm{~mm}$ (see his Figure 6).

Observational and theoretical uncertainties obfuscate the relationship between a measured submillimeter continuum slope and the functional form of the opacity in a disk. Overcoming these difficulties to pursue evidence of the collisional agglomeration of dust grains in the earliest stages of planet formation will at least require better observations, including resolved images at wavelengths extending beyond $\sim 1 \mathrm{~mm}$ (where the emission is more optically thin; e.g., Testi et al. 2001), flux measurements near the SED turnover point (in the 100 to $300 \mu \mathrm{m}$ range), and studies of solid-state dust emission features in the mid-infrared (e.g., van Boekel et al. 2004). However, our results leave no doubt that the measured submillimeter continuum slopes for YSOs are significantly less than those noted for molecular clouds, where $\alpha \approx 4$.

\subsection{Connections to Stellar Properties}

The physical properties of a young star and its circumstellar disk could be related due to their mutual formation and subsequent gravitational and thermal links. Generally, observational indications of any such relationship are absent, presumably due to a wide range of initial circumstellar conditions for individual sources and the relatively small ranges of stellar masses and ages. As with Beckwith et al. (1990), we do not find any correlations between measured submillimeter properties and any characteristic of the stellar photosphere (e.g., effective temperature, luminosity, optical

fluxes or colors, etc.). One perhaps notable exception is the large value of $T_{1}$ derived for the two A 
stars in this sample (AB Aur and V892 Tau). Natta, Grinin, \& Mannings (2001) also suggest that hotter stars have generally higher dust temperatures in their disks, but there is no noticeable trend for the cooler ( $\mathrm{K}$ and $\mathrm{M}$ ) majority of this sample. This disconnect between the stellar photosphere and the outer disk, where the submillimeter emission is generated, is not surprising. Despite the increase in vertical scale height of the disk with radius expected from hydrostatic equilibrium (e.g., Kenyon \& Hartmann 1987), radiative transfer models for structurally realistic disks indicate that the bulk of the submillimeter emission comes from the dust near the disk midplane, and not in the flared atmosphere which can be more directly affected by the stellar photosphere (Chiang \& Goldreich 1997, 1999).

The gravitational link between a young star and its disk suggests that the stellar and disk masses may be related. Circumstellar disks are self-gravitationally stable if their mass is less than a fraction (a few tenths) of the stellar mass (Shu et al. 1990; Laughlin \& Bodenheimer 1994). In principle, this could allow more massive stars to harbor more massive disks. Natta, Grinin, \& Mannings (2001) combine interferometric measurements of disks around early-type stars with the $1.3 \mathrm{~mm}$ survey of Beckwith et al. (1990) and claim a marginal correlation between the disk mass and stellar mass $\left(M_{*}\right)$ over 2 orders of magnitude in $M_{*}$ for roughly 100 objects (however, see Mannings \& Sargent 2000), although the dispersion is substantial. To revisit this issue, we have collected optical/near-infrared magnitudes and spectral classifications from various sources in the literature (Cohen \& Kuhi 1979; Jones \& Herbig 1979; Slutskiü, Stal'bovskiü, \& Shevchenko 1980; Herbig, Vrba, \& Rydgren 1986; Herbig \& Bell 1988; Strom et al. 1989; Hartmann et al. 1991; Gomez et al. 1992; Bouvier et al. 1993; Briceño et al. 1993; Hartigan, Strom, \& Strom 1994; Martín et al. 1994; Kenyon \& Hartmann 1995; Hernández et al. 2004; White \& Hillenbrand 2004, spectral types are listed in Table 1). A consistent set of visual extinctions was determined from the $(V-I)$ color excesses, using the intrinsic colors tabulated by Kenyon \& Hartmann (1995) and the interstellar extinction law derived by Cohen et al. (1981). De-reddened visual magnitudes and spectral types were converted to bolometric luminosities and effective temperatures again using the intrinsic values of Kenyon \& Hartmann (1995). Stellar masses and ages were determined by reference to theoretical pre-main-sequence evolution tracks and isochrones (D'Antona \& Mazzitelli 1997) in a Hertzsprung-Russell diagram.

As Figure 9 demonstrates, there are no correlations between the submillimeter properties listed in Table 1 and stellar mass or age, but the ranges of those stellar properties (see Figure 1) may be too limited to infer a direct evolutionary sequence. However, the upper right panel of this figure shows that the region corresponding to higher mass disks at late times ( $\geq 6 \mathrm{Myr}$ ) is significantly depopulated. While there are not many objects in Taurus-Auriga with such ages, this unoccupied region in the diagram is consistent with other studies that indicate disk fractions approaching zero in the 6 to $10 \mathrm{Myr}$ age range (e.g., Haisch, Lada, \& Lada 2001). Figure 10 shows the cumulative distributions of the mass ratio of disk to star, constructed with the Kaplan-Meier estimator. Log-normal distributions provide poor fits in this case, but these distributions are fit fairly well with power laws of index between -1.5 and -2 for mass ratios larger than $\sim 10^{-3}$. The 
median disk to star mass ratio is $0.5 \%$. The fraction of disks which may be self-gravitationally unstable (mass ratios larger than $\sim 0.1$ ) is negligible in Taurus-Auriga: roughly $6 \%$, which itself may be an overestimate due to envelope emission for some of the Class I objects at the high end of the distribution. However, if $\beta=2$ is more appropriate, then the fraction of unstable disks can be as high as one third. A small fraction of objects (a few percent) has a mass ratio less than $10^{-3}$.

\subsection{The Effects of Multiplicity}

The evolution of circumstellar disks can be dictated by either internal (e.g., viscous accretion, gravitational instability, planet formation) or external processes. Examples of the latter include ultraviolet photoevaporation in the vicinity of a massive star (e.g., Johnstone, Hollenbach, \& Bally 1998), dynamical interactions with other stars in a crowded cluster environment (Kroupa 1995; Boffin et al. 1998) or in a local multiple star system. In the low stellar density Taurus-Auriga region, which is devoid of stars earlier than A0, the dominant external process affecting disk evolution is expected to be dynamical star-disk or disk-disk interactions in multiple star systems. Most young stars in nearby clusters and main-sequence stars in the field are in multiple systems, and the multiplicity fraction in Taurus-Auriga may be exceptionally large (Mathieu 1994; Mathieu et al. 2000). The similar multiplicity fractions for YSOs and main-sequence field stars indicates that binary formation occurs early in stellar evolution (at least before the Class II stage, and likely much earlier), when significant circumstellar material is still present. Gravitational interactions are expected to severely affect the structural integrity of disks in the system, including truncation of the outer parts of individual circumstellar disks, gap formation in circumbinary disks, or even complete dissipation of circumstellar material via accretion or ejection (Artymowicz \& Lubow 1994).

As an example, consider a young binary system with semimajor axis $a$ and eccentricity $e$, which also harbors two individual circumstellar disks and a larger circumbinary disk. Simulations of the gravitational dynamics in such a system indicate that the circumstellar disks will be truncated and a gap will open in the circumbinary disk, at radii which are determined primarily by the values of $a$ and $e$ (Artymowicz \& Lubow 1994). The disk model described in $\S 3.1$ can be adjusted to determine the effects on the SED of such disk configurations by re-setting the inner and outer radii for the various disk components or simply by setting $\Sigma_{r}=0$ for the cleared regions (e.g., Jensen, Mathieu, \& Fuller 1996). One expected result from these SED models is that the submillimeter emission should be significantly diminished for systems with a projected semimajor axis $\left(a_{p}\right)$ on the order of a few tens of AU, but essentially identical to single stars for small and large $a_{p}$. Previous observations have indicated that $a_{p} \lesssim 50-100$ AU binaries have less submillimeter emission than single stars or wider binaries (Jensen, Mathieu, \& Fuller 1994, 1996; Osterloh \& Beckwith 1995), with the important exception of spectroscopic binaries ( $a \lesssim 1$ AU; e.g., Mathieu et al. 1995, 1997).

Table 3 gives a list of multiple stars in the sample and their projected separations. This information and the data in Table 1 have been compiled in Figure 11, which shows the $850 \mu \mathrm{m}$ flux density and disk mass as a function of projected semimajor axis. To be consistent with previous 
work, spectroscopic binaries as well as Class I sources have been excluded in this figure and the analysis that follows. Unresolved higher-order multiple star systems ( $\geq 3$ stars) without resolved observations in the literature were assigned the same $F_{\nu}$ or $M_{d}$ value for all projected separations. Notes on assigning values for a few other systems are provided in Table 3. We utilize a variety of two-sample statistical tests that incorporate upper limits to determine the probabilities that the $850 \mu \mathrm{m}$ flux densities and disk masses in various binary subsamples are drawn from different parent distributions. The total sample is separated into categories based on projected semimajor axis, resulting in three groups: close binaries with separations less than some critical value, $a_{c}$; wide binaries with separations larger than $a_{c}$; and single stars.

Table 4 lists the ranges of probabilities that the various subsamples for $a_{c}=50 \mathrm{AU}$ and $100 \mathrm{AU}$ differ from a sequence of survival analysis statistical tests performed with the ASURV software: the logrank, Peto \& Peto, Peto \& Prentice, and Gehan tests (see the detailed descriptions by Feigelson \& Nelson 1985). The same tests were also performed for Class II objects only. The results in Figure 11 and Table 4 confirm the earlier conclusions of Jensen, Mathieu, \& Fuller (1994, 1996): submillimeter flux densities and disk masses are significantly lower in close binaries $\left(a_{p} \leq 50-100 \mathrm{AU}\right)$ than wider or isolated systems and wide binaries essentially have the same disk masses as single stars. These differences are greatest for a critical semimajor axis $a_{c}=100 \mathrm{AU}$. The results for the total sample (i.e., when Class III binaries are included) generally exhibit lower probabilities than the subsample of only Class II objects in Table 4, with the exception of the close and wide binary populations with the $a_{c}=100 \mathrm{AU}$ cutoff criterion. These differences are likely due to the evolutionary behavior of disks between the Class II and III stages (see $\S 4$ ), rather than an environmental effect in the multiple system. The exact probabilities for the various subsamples appear to be fairly sensitive to the assignment of flux densities or disk masses in unresolved higherorder multiple systems. High resolution interferometric observations are needed to determine the relative submillimeter contributions of individual components in these systems.

In a statistical sense, the presence of a companion in the range of $\sim 1-100 \mathrm{AU}$ decreases the apparent disk mass(es) in the system, presumably due to enhanced accretion onto the stars and/or dispersal into the local ISM. However, multiple star systems still contain disks, as evidenced by the relatively high detection rate in the submillimeter, $66 \pm 10 \%$ for multiple systems compared to $58 \pm 8 \%$ for single stars, as well as other inner disk signatures in the optical and infrared (e.g., White \& Ghez 2001). High-resolution interferometric measurements of disks in multiple systems have revealed a number of important exceptions to the statistical analysis above. For example, the GG Tau A and UZ Tau systems both have small projected separations but very large disk masses, the former in a circumbinary disk and the latter in a pair of disks with four stellar components (Koerner, Sargent, \& Beckwith 1993a; Dutrey, Guilloteau, \& Simon 1994; Jensen, Koerner, \& Mathieu 1996). Moreover, single-dish continuum surveys may be missing signatures of outer disks in close multiple systems: a close binary $\left(a_{p}=32 \mathrm{AU}\right)$ in the SR 24 triple system in Ophiuchus was found to have a large circumbinary gas disk detected in CO line emission but not in the continuum due to its low mass (Andrews \& Williams 2005). The question of whether the large fraction of 
young stars in multiple systems could eventually harbor planetary systems will remain unanswered until more detailed case studies with interferometers (e.g., Jensen \& Akeson 2003) can confirm the properties of their disks.

\section{Discussion}

A summary of representative numbers derived from this submillimeter survey of Taurus-Auriga is provided in Table 5. Listed are submillimeter detection fractions as well as median values and standard deviations of disk masses and submillimeter continuum slopes for the total sample and various subsamples of interest. Of the complete sample of 153 YSOs, $61 \pm 6 \%$ were detected for at least one submillimeter frequency, with a median $M_{d} \approx 5 \times 10^{-3} \mathrm{M}_{\odot}$ and $\alpha \approx 2.0$. Single and multiple star systems have essentially identical detection rates and similar continuum slopes. However, as discussed in detail in the previous section, closer binaries have statistically lower disk masses than wider systems or single stars. Although Figure 12 demonstrates that there is no direct correlation between $M_{d}$ or $\alpha$ and the equivalent width or luminosity of the $\mathrm{H} \alpha$ emission line, there is an obvious difference in the submillimeter detection fraction between WTTS and CTTS disks. The very high submillimeter detection rate for CTTS disks $(91 \pm 11 \%)$ is consistent with all CTTSs having disk masses greater than $\sim 10^{-4} \mathrm{M}_{\odot}$. The bulk of the detected WTTS disks are clustered near $W(\mathrm{H} \alpha)=10 \AA$ : when the WTTS/CTTS division criterion is slightly relaxed, this result suggests that nearly all WTTSs are either diskless or have very low disk masses. Therefore, the equivalent width of the $\mathrm{H} \alpha$ emission line appears to be a fairly robust predictor of the presence of a "massive" disk.

Spectral energy distribution classifications of objects in the sample were determined based on power-law fits from 2 to $60 \mu \mathrm{m}$ (when possible) with data from the literature (Strom et al. 1989; Weaver \& Jones 1992; Kenyon \& Hartmann 1995; Hartmann et al. 2005, and references therein). We adopt the classification breakdown of Greene et al. (1994), using the values of the powerlaw index $n$ (defined by $\nu F_{\nu} \propto \nu^{n}$ ) to distinguish between Class I, Flat Spectrum, Class II, and Class III sources. The derived classifications are listed in Table 1. Although it is not absolutely calibrated in time, the YSO evolution sequence defined by the shape of the infrared SED is certainly indicative of changes in the physical structure of the inner regions of the circumstellar disk and/or envelope. With the large sample of submillimeter data presented above, we can address the issue of corresponding changes in the physical properties of the outer disk.

Motivated by the differences in the detection rates and median properties of the various SED

classes listed in Table 5, the same survival analysis two-sample statistical tests used in $\S 3.4$ were employed to determine the probabilities that the $850 \mu \mathrm{m}$ flux densities, inferred disk masses, and submillimeter continuum slopes for various SED and $\mathrm{H} \alpha$ line strength classes are drawn from different parent populations. The test results are given in Table 6, and the cumulative distributions of $F_{\nu}, M_{d}$, and $\alpha$ for different classes are shown in Figure 13. There are statistically significant progressions of decreasing submillimeter flux densities, disk masses, and continuum slopes along 
the infrared SED evolution sequence. Flat Spectrum objects fit between Class I and II objects in these respects, with somewhat more similarity to the latter. Incorporating the Flat Spectrum objects with either the Class I or II objects does not make any significant difference in these results. Apparently the properties of the outer disk/envelope evolve along a similar evolutionary sequence as the inner disk.

Figure 13 clearly shows that Class I objects have significantly larger submillimeter flux densities, disk masses, and continuum slopes than Class II objects. It should again be stressed that the extent to which these values are representative of Class I disks, rather than disks + inner envelopes, is questionable. It has been suggested that many Class I disk properties could be mimicked by a Class II disk viewed at high inclination (e.g., Chiang \& Goldreich 1999; White \& Hillenbrand 2004). It is shown in Figure 14 that for a given mass, a high inclination angle produces both a lower flux density and a lower continuum slope; the opposite is seen in Figure 13 and Table 6. If the Class I emission is primarily from a disk (with only a comparatively small contribution from the inner envelope), a simple re-orientation of a Class II disk will not reproduce the Class I submillimeter properties without additional changes in mass, temperature, or opacity. The distributions found here of the empirical (model-independent) flux densities and continuum slopes corroborate the original picture of Class I sources as disk + envelope systems: the higher flux densities and "disk" masses may be due to additional envelope mass, and the higher continuum slopes may be due to the less-processed (i.e., lower amount of grain growth) dust in the envelope. A large interferometric sample will be required to definitively settle the issues involved in a comparison of Class I and II disks.

Unfortunately, there are no measurements of a submillimeter continuum slope for any of the Class III objects in the sample, and the 3- $\sigma$ upper limits are too large to make any definitive statements on an evolutionary trend in $\alpha$. The direct relationships between $M_{d}$ or $\alpha$ and the infrared SED slope are shown in Figure 15. There is no direct correlation with $M_{d}{ }^{4}$ but differences between the SED classes in general are apparent. A more steady decrease in $\alpha$ is seen across the evolution sequence, which if it continues would imply very shallow continuum slopes for Class III disks $(\alpha \sim$ $1-1.5)$. The Spearman rank correlation coefficient in this case is -0.50 , with a $99.98 \%$ confidence level $(3.7-\sigma)$. The best-fit linear relation between the submillimeter and infrared continuum slopes is $\alpha=0.40( \pm 0.04) n+2.09( \pm 0.03)$. This trend can not be explained solely by decreasing optical depths in the disks along an evolutionary sequence: lower optical depths produce steeper continuum slopes. Another effect, such as a shallow opacity function or temperature/surface density evolution, must be acting to decrease $\alpha$ in this manner. However, interferometric observations of the Class I sources at several wavelengths would be required to confirm the validity of this trend.

The submillimeter detection fraction and the fraction of objects with a near-infrared $\left(K_{s}-L\right)$

\footnotetext{
${ }^{4}$ A significant correlation exists when L1551 IRS 5 and L1551 NE are included (the two points in the upper left corner), but these objects likely have large contributions to $M_{d}$ from their envelopes, and so have been excluded in this part of the analysis.
} 
excess are identical: $60 \pm 7 \%{ }^{5}$ Figure 16 is a near-infrared color-color diagram that indicates the sources with submillimeter detections. Of the 6 sources with infrared excesses that were not detected in the submillimeter, 5 could have anomalous colors due to mismatched photometry and/or infrared companions (see the Appendix). Three of the 55 sources with essentially no near-infrared excess, or $5 \pm 3 \%$, were detected in the submillimeter: GM Aur, V836 Tau, and CoKu Tau/4. ${ }^{6}$ All three of these YSOs also have mid- and far-infrared emission, indicating that the lack of near-infrared excess may be due to a clearing of dust in the inner $\sim 1 \mathrm{AU}$ of their disks. In addition to these "transition" objects, three Class III sources, V807 Tau, FW Tau, and LkH $\alpha 332 / \mathrm{G} 1$, were also detected in the submillimeter (a $5.6 \pm 3.2 \%$ detection rate), along with another possible Class III candidate whose SED classification remains to be confirmed due to lack of infrared data (HQ Tau). However, in general a YSO with a near-infrared excess also has submillimeter emission consistent with a disk mass greater than $\sim 10^{-4} \mathrm{M}_{\odot}$, and vice versa. The small fraction of objects, less than $10 \%$, with evidence for an outer disk (from submillimeter data) and no inner disk suggests that the timescale for the disappearance of both infrared and submillimeter disk emission is relatively short; no more than a few hundred thousand years (i.e., $\lesssim 10 \%$ of the typical YSO age in Taurus-Auriga). In agreement with the comparatively low detection fraction for WTTS disks (16 $\pm 5 \%)$ and other similar analyses (e.g., Skrutskie et al. 1990; Wolk \& Walter 1996; Duvert et al. 2000), these results imply that the inner and outer disk dissipate, or become unobservable, almost simultaneously.

The physical mechanism responsible for the rapid and essentially radially constant "disappearance" timescale remains to be explained. Viscous accretion onto the central star alone does not readily produce the apparently rapid inner-outer disk dissipation (Hollenbach, Yorke, \& Johnstone 2000). In fact, evolution under accretion processes predicts only small changes in submillimeter emission with time (e.g., Hartmann et al. 1998). Models which incorporate the ultraviolet photoevaporation of the outer disk along with viscous accretion have more success in reproducing the inferred dissipation timescale, particularly for disk emission out to $\sim 100 \mu \mathrm{m}$ (Clarke, Gendrin, \& Sotomayor 2001; Armitage, Clarke, \& Palla 2003). However, these "ultraviolet switch" models also suggest that submillimeter emission is relatively unaffected, and could therefore predict a fairly large fraction of WTTSs or Class III sources with submillimeter emission. Clarke, Gendrin, \& Sotomayor (2001) suggest that the low observed fraction of such transition objects noted by Duvert et al. (2000) and confirmed by the larger sample presented here may be accomodated in their models if different surface density profiles or viscosity values are adopted.

An alternative explanation to actually losing disk material, onto the star or elsewhere, is a process which renders the dust invisible to conventional observations. A compelling possibility is

\footnotetext{
${ }^{5}$ The values given here and in Table 5 are slightly different because a small fraction of the objects in the sample do not have $L$-band measurements in the literature.

${ }^{6} \mathrm{~A}$ fourth source, BP Tau, appears to fit in this category in Figure 16. However, this is likely due to mismatched photometry from 2MASS and the literature, because BP Tau has a clear excess in homogeneous datasets, e.g. Kenyon \& Hartmann (1995).
} 
the collisional agglomeration of dust grains in the disk. Accelerated by gravitational settling to the disk midplane, the characteristic grain growth timescales even at fairly large disk radii are thought to be shorter than the transition timescale inferred above (e.g., Weidenschilling \& Cuzzi 1993). Perhaps the grain growth process has rendered the disks around many of the evolved (e.g., Class III) sources invisible by creating a significant population of large $(\sim \mathrm{cm}$-sized $)$ grains which are inefficient emitters at both infrared and submillimeter wavelengths. If this is to be the case, any collisional fragmentation process of the aggregate grains should not produce more than $\sim 10^{-4} \mathrm{M}_{\odot}$ of particles which are efficient submillimeter emitters. The shallow submillimeter slopes measured in $§ 3.3$ and the implied low values of the opacity index $\beta$ lend some credibility to the grain growth argument. Theoretical studies indicate that $\beta$ values such as those inferred in this sample $(\beta \sim 1$ or less) can be the result of a significant population of large grains (Miyake \& Nakagawa 1993; Pollack et al. 1994; Dullemond \& Dominik 2005). The collisional growth of dust grains has also been inferred from submillimeter observations of both young (e.g., Beckwith \& Sargent 1991; Mannings 1994; Koerner, Chandler, \& Sargent 1995) and old (e.g., Calvet et al. 2002; Hogerheijde et al. 2003) low mass disks, and particularly for those around the more massive Herbig Ae stars (Testi et al. 2001, 2003; Natta et al. 2004). Complementary studies of scattered light (e.g., McCabe, Duchêne, \& Ghez 2003; Duchêne et al. 2004b) and mid-infrared spectra (Meeus et al. 2003; Przygodda et al. 2003; van Boekel et al. 2004; Kessler-Silacci et al. 2005) also suggest that typical grain sizes are larger in these disks than for the ISM. The feasibility of this hypothesis depends critically on coupling with another mechanism which can diminish the accretion of gas, and therefore also explain the low submillimeter detection fraction for WTTSs.

The distribution of $M_{d}$ shown in Figure 5 indicates that typical disks have masses significantly lower than those required by two of the leading theoretical models for giant planet formation. Both the core accretion (Pollack et al. 1996) and disk instability (Boss 1998) scenarios require disk masses at least a few times that of the MMSN to form a Jupiter-like planet; roughly an order of magnitude higher than the median mass inferred for Taurus-Auriga disks. Radial velocity surveys suggest that roughly $10 \%$ of stars harbor a gas giant planet within a few AU (e.g., Marcy et al. 2005), with the prospect that better sensitivity to long-period planets could significantly increase that fraction (e.g., Fischer et al. 2001). This shows that planet formation is a fairly common process. In order for that to be the case, the disk mass distribution constructed in $\S 3.2$ needs to be reconciled with the theoretical requirements of the planet formation models. Possible remedies could be extracted from changes to the simple disk model used in $\S 3$ : for example, adjustments to the disk surface density profile, or a significant decrease in the normalization of the opacity function. Unfortunately, solutions like these will remain untested until more advanced observations become available (e.g., interferometers with $\sim 0^{\prime \prime} 1$ spatial resolution). A likely alternative explanation, as discussed above, is that a significant fraction of the disk mass is locked up in large grains or planetesimals which are inefficient emitters at submillimeter wavelengths. 


\section{Summary}

We have conducted a sensitive, multiwavelength submillimeter survey of 153 Taurus-Auriga YSOs in an effort to analyze properties of the outer regions of circumstellar dust disks. Some of the key results from this survey are summarized here:

- The disk mass (or submillimeter flux density) distribution function is well matched with a log-normal distribution centered around $5 \times 10^{-3} \mathrm{M}_{\odot}$ with a large dispersion $(0.5 \mathrm{dex})$. The vast majority of disks in Taurus-Auriga have substantially lower masses than is thought to be required for giant planet formation. However, a significant fraction of the disk mass could be stored in large grains or planetesimals which do not contribute to the submillimeter emission.

- We provide the largest set of submillimeter continuum slope measurements of YSOs to date. The empirical behavior of the continuum from $350 \mu \mathrm{m}$ to $1.3 \mathrm{~mm}$ is well-described by $F_{\nu} \propto$ $\nu^{2.0 \pm 0.5}$, which is much flatter than for the interstellar medium. The low observed slope values are probably due to a combination of optical depth effects (Beckwith \& Sargent 1991) and an inherently shallow opacity function from the top-heavy grain size distribution produced by collisional agglomeration of material in the disk.

- There do not appear to be any links between stellar and disk properties in the sample, although the stellar masses and ages span a relatively limited range. The median disk to star mass ratio is $\sim 0.5 \%$.

- Submillimeter flux densities and disk masses are statistically lower for stars with close companions (projected semimajor axes less than $\sim 100 \mathrm{AU}$ ) than for wider binaries or single stars. However, multiple star systems often still contain disks, regardless of their projected separations. Multiple star systems with wider separations have flux densities and disk masses comparable to single stars.

- In general, the standard signatures of the inner disk (e.g., accretion diagnostics or infrared excess emission) are accurate predictors of a disk mass greater than $\sim 10^{-4} \mathrm{M}_{\odot}$.

- Statistically significant changes in the distribution functions of submillimeter flux densities, disk masses, and continuum slopes are found for the progressive stages of YSO evolution inferred from inner disk observations. These measured outer disk properties decrease from Class $\mathrm{I} \rightarrow \mathrm{II} \rightarrow$ III objects as well as for CTTSs $\rightarrow$ WTTSs. The implication is that the inner and outer disk develop along a similar evolutionary sequence. A multiwavelength interferometric survey of Class I objects would be very useful for determining the relative contributions of a disk and inner envelope in these systems for a more sophisticated comparison with their presumably more evolved counterparts (Class II and III objects).

- Only a small fraction of objects $(<10 \%)$ which have no inner disk signatures were detected in the submillimeter, suggesting that both infrared and submillimeter disk emission disappear on 
a similar timescale (within $\sim 10^{5}$ years of each other). There are two timescales in operation for disk evolution: (1) the relatively long ( $\sim 5$ to $10 \mathrm{Myr}$ ) lifetime of Class II/CTTS disks, and (2) the rapid (a few $\times 10^{5}$ years) transition period to Class III/WTTS disks. Understanding the mechanisms responsible for these timescales, particularly the trigger for the transition stage, remain key problems in disk evolution. Some possible explanations for the essentially radiallyindependent disk dissipation timescale include viscous accretion with photoevaporation by the central star (e.g., Clarke, Gendrin, \& Sotomayor 2001) or rapid grain growth in the early stages of planet formation (e.g., Weidenschilling \& Cuzzi 1993).

We acknowledge useful conversations, suggestions, and advice from Michael Liu, Lee Hartmann, Ted Simon, and Alan Boss. We would like to thank the JCMT and CSO support staffs, and in particular Colin Borys, for their assistance. An anonymous referee provided valuable criticism which helped improve this paper. This work was supported by NSF grant AST-0324328. This research has made use of the JCMT archive at the Canadian Astronomy Data Center, which is operated by the Dominion Astrophysical Observatory for the National Research Council of Canada's Herzberg Institute of Astrophysics and the NASA/IPAC Infrared Science Archive, which is operated by the Jet Propulsion Laboratory, California Institute of Technology, under contract with the National Aeronautics and Space Administration.

\section{A. Comments on Disk Models}

A simple disk structure model was presented in $§ 3.1$ and used to generate SEDs of thermal dust emission and derive a relationship between the submillimeter flux density and disk mass. A lack of SED data severely limits the number of degrees of freedom in this modeling, and therefore a number of parameters were fixed: inclination, inner and outer radii, and the power law indices of the radial surface density and frequency spectrum of the opacity. Here we determine the effects that reasonable alternative values for these fixed parameters would have on the $F_{\nu}-M_{d}$ relationship. Readers would also benefit from the mathematical formalism discussed in detail by Beckwith et al. (1990). The analysis is done comparatively, relative to a fiducial disk model based on the median values discussed in the text: $i=0^{\circ}, p=1.5, T_{1}=150 \mathrm{~K}, q=0.6, r_{\circ}=0.01 \mathrm{AU}, R_{d}=100 \mathrm{AU}$, and $\beta=1$. A set of $850 \mu \mathrm{m}$ flux densities was computed using Equation 2 for a grid of disk masses with this fiducial parameter set excepting one of the previously fixed variables. The flux density - disk mass relationships for various values of $p, q, r_{\circ}, R_{d}, T_{1}$, and $\beta$ are shown in Figure 17. It should be noted that these plots are only intended to illustrate the effects of changing a single parameter in these models. In reality, the parameters are usually somewhat coupled, and therefore changes

in one parameter affect others: such coupled effects are not considered in this simple comparative analysis.

Larger values of $q, r_{\circ}, R_{d}$, and $\beta$ all result in a larger disk mass for a given flux density. The 
middle panels in Figure 17 indicate that the disk boundaries play only a minor role in setting the $F_{\nu}-M_{d}$ relationship. The roles of the opacity index and temperature normalization are roughly those expected from the optically thin assumption given in Equation 1, where $F_{\nu} \propto \kappa_{\nu} M_{d} T^{-1}$. For low-mass disks (i.e., $M_{d} \lesssim 10^{-2} \mathrm{M}_{\odot}$ ), the shape of the radial surface density profile can have a significant effect on the submillimeter flux density because most of that emission is optically thin. For the same reason, the radial temperature behavior dictated by $q$ has an opposite effect and impacts the flux densities for the more massive, optically thick disks. The largest relative deviations in the flux density - disk mass relationship are set by the parameters which describe the radial temperature profile; $T_{1}$ and $q$. Fortunately, the parameters of the temperature profile can be reliably determined for individual disks using observations in the mid- and far-infrared. Sensitive observations with the Spitzer Space Telescope will essentially ensure that the opacity is the single dominant uncertainty in the determination of a disk mass from submillimeter observations.

In Figure 18, the same procedure as above was used to examine the effects of different fixed parameter choices on the relationship between the submillimeter continuum slope and the disk mass (note that here we have fixed $\beta=1$ as a reference value). Once again, the disk boundaries play a negligible role in the relationship. The radial surface density index has only a small impact on the $\alpha-M_{d}$ relationship in general, although a constant surface density disk $(p=0)$ gives a roughly constant submillimeter continuum slope (and lower than for larger values of $p$ ) for low-mass disks. The parameters of the temperature profile again show the largest deviations from the fiducial $\alpha-M_{d}$ relationship. The effects of a changing $\beta$ in this relationship are also large. See Beckwith \& Sargent (1991) and Mannings \& Emerson (1994) for a mathematical description of what is shown in these plots.

An alternative view of the relationship between the observed continuum slope $(\alpha)$ and the power law index of the frequency behavior of the opacity $(\beta)$ can be obtained directly from these models. Figure 19 shows computed continuum slopes as a function of $\beta$ for various disk masses. The relationship is essentially linear for $M_{d} \lesssim 0.1 \mathrm{M}_{\odot}$ with slopes of nearly unity independent of disk mass, but significantly different intercept values. All of the curves fall below the nominal $\beta=\alpha-2$ line which is representative of optically thin emission in the Rayleigh-Jeans limit. This effect is due to the failure of the Rayleigh-Jeans limit and the fraction of optically thick submillimeter emission, which can be fairly high at the shortest wavelengths (Beckwith et al. 1990; Beckwith \& Sargent 1991; Mannings \& Emerson 1994). This synthetic grid illustrates the conclusion in $\S 3.3$ that the roughly gaussian distribution of $\alpha$ centered around $\sim 2$ suggests that the opacity index is likely between $\sim 0.5$ and 1.5, and certainly less than the ISM value of 2 unless the disk masses are severely underestimated.

Figure 20 was generated from the fiducial model (the top panel also assumes the median $M_{d}=0.005 \mathrm{M}_{\odot}$ ) described above to illustrate that the assumption of optically thin emission in the submillimeter continuum is not always valid (as pointed out by Beckwith et al. 1990). The disk becomes optically thin at the radius, $r_{1}$, where $\tau_{\nu}=\kappa_{\nu} \Sigma_{r_{1}}=1$. That criterion can be solved to 
determine $r_{1}$ using Equation 4 and the relationship between $\Sigma_{\circ}$ and $M_{d}$, giving

$$
r_{1}=\left(\kappa_{\nu} \Sigma_{\circ}\right)^{1 / p} r_{\circ}=\left[\frac{(2-p) \kappa_{\nu} M_{d}}{2 \pi\left(R_{d}^{2-p}-r_{\circ}^{2-p}\right)}\right]^{1 / p},
$$

when $p \neq 2$; inserting the parameters fixed in $\S 3.1$ gives $r_{1} \approx 184\left(M_{d} / \mathrm{M}_{\odot}\right)^{2 / 3} \mathrm{AU}$. The fraction of the submillimeter flux density from optically thick emission, $\Delta$ (defined as the ratio of the flux density from $r<r_{1}$ to the total flux density: see Beckwith et al. 1990) increases exponentially until $\Delta=1$ around 0.4 to $1 \mathrm{Jy}$, depending on the wavelength. Even faint submillimeter sources have $\sim 15 \%$ of their flux densities generated in the innermost (radially), optically thick regions of the disk. In terms of the fraction of the disk mass which gives rise to optically thick emission (the ratio of the integrated surface density from $r_{\circ}$ to $r_{1}$ to the total disk mass), a more gradual trend with disk mass is present. Roughly $25 \%$ of the mass in a MMSN disk with the fiducial parameter set contributes optically thick emission in the submillimeter. Comparison of the right panel in Figure 20 with the information in Figures 4 and 8 demonstrate the effects these relatively high optical depths have on estimating $M_{d}$ and $\alpha$ (and subsequently $\beta$ ) from observations.

\section{B. Comments on Individual Sources}

There are six objects in the sample with apparent near-infrared excesses, but no detected submillimeter emission: DP Tau, JH 223, CoKu Tau/3, FV Tau/c, CZ Tau, and CIDA-3. Three of these six (CoKu Tau/3, FV Tau/c, and CZ Tau) are multiple stars (see Table 3), introducing the possibility that the near-infrared excess is exaggerated by a red companion. The infrared colors of JH 223, CoKu Tau/3, CZ Tau, and CIDA-3 also seem questionable in light of the fact that these sources are WTTSs. Therefore, multiplicity and/or mismatched photometry from the literature for WTTSs could explain why submillimeter emission was not detected for most of these sources. The non-detection of DP Tau is more puzzling.

As mentioned in $\S 4$, there are three sources without near-infrared excesses which are detected in the submillimeter (and mid- and far-infrared): GM Aur, V836 Tau, and CoKu Tau/4. Supplementary to these transition objects are the Class III sources detected at $850 \mu \mathrm{m}$ : V807 Tau, FW Tau, and $\mathrm{LkH} \alpha 332 / \mathrm{G} 1$. These objects have presumably evacuated the inner portions of their disks, perhaps due to clearing from the planet formation process (see, e.g., Forrest et al. 2004, regarding $\mathrm{CoKu} \mathrm{Tau} / 4)$. Examinations of the SEDs for this sample highlight some other interesting sources in terms of possible near-infrared deficits: FM Tau, FQ Tau, UX Tau, and HK Tau. Seven late-type (excluding V892 Tau and SU Aur) WTTS disks are detected in the submillimeter: V773 Tau, IQ Tau, UX Tau, IT Tau, CoKu Tau/4, LkH $\alpha$ 332/G1, and V836 Tau. A number of other sources detected in the submillimeter but with very little information between $\sim 2$ and $850 \mu$ m include FY Tau, GN Tau, CIDA-7, CIDA-8, CIDA-9, and HQ Tau. These are prime targets for mid- and far-infrared observations from both the ground and the Spitzer Space Telescope. One final source worth further investigation is CY Tau. This object has an unique SED, well-described by a shallow 
power law from $\sim 2 \mu \mathrm{m}$ out to at least $1.3 \mathrm{~mm}$. The models in $\S 4$ are clearly inappropriate for this case, as they predict very large disk masses $\left(M_{d} \sim 10 \mathrm{M}_{\odot}\right)$. Updated mid- and far-infrared photometry and spectroscopy of this source, along with resolved observations of the dust content may clear up the true nature of the circumstellar material.

\section{REFERENCES}

Adams, F. C., \& Shu, F. H. 1986, ApJ, 308, 836

Adams, F. C., Lada, C. J., \& Shu, F. H. 1987, ApJ, 312, 788

Adams, F. C., Emerson, J. P., \& Fuller, G. A. 1990, ApJ, 357, 606

Aikawa, Y., van Zadelhoff, G.-J., van Dishoeck, E. F., \& Herbst, E. 2002, A\&A, 622, 386

Akeson, R. L., et al. 2005, ApJ, 622, 440

Andrews, S. M., \& Williams, J. P. 2005, ApJ, 619, L175

Armitage, P. J., Clarke, C. J., \& Palla, F. 2003, MNRAS, 342, 1139

Artymowicz, P., \& Lubow, S. H. 1994, ApJ, 421, 651

Beckwith, S. V. W., Sargent, A. I., Chini, R. S., \& Güsten, R. 1990, AJ, 99, 924

Beckwith, S. V. W., \& Sargent, A. I. 1991, ApJ, 381, 250

Beckwith, S. V. W., \& Sargent, A. I. 1993, ApJ, 402, 280

Beckwith, S. V. W., Henning, T., \& Nakagawa, Y. 2000, in Protostars \& Planets IV, eds. V. Mannings, A. P. Boss, \& S. S. Russell (Tucson: Univ. Arizona Press), 533

Boffin, H. M. J., Watkins, S. J., Bhattal, A. S., Francis, N., \& Whitworth, A. P. 1998, MNRAS, 300,1189

Boss, A. P. 1998, ApJ, 503, 923

Bouvier, J., Cabrit, S., Fernández, M., Martín, E. L., \& Matthews, J. M. 1993, A\&A Suppl. Ser., 101,485

Briceño, C., Calvet, N., Gomez, M., Hartmann, L. W., Kenyon, S. J., \& Whitney, B. A. 1993, PASP, 105, 686

Calvet, N., \& Gullbring, E. 1998, ApJ, 509, 802

Calvet, N., D’Alessio, P., Hartmann, L., Wilner, D., Walsh, A., \& Sitko, M. 2002, ApJ, 568, 1008 
Chandler, C. J., \& Richer, J. S. 2000, ApJ, 530, 851

Chiang, E., Phillips, R. B., \& Lonsdale, C. J. 1996, AJ, 111, 355

Chiang, E. I., \& Goldreich, P. 1997, ApJ, 490, 368

Chiang, E. I., \& Goldreich, P. 1999, ApJ, 519, 279

Chiang, E. I., Joung, M. K., Creech-Eakman, M. J., Qi, C., Kessler, J. E., Blake, G. A., \& van Dishoeck, E. F. 2001, ApJ, 547, 1077

Chini, R., Ward-Thompson, D., Kirk, J. M., Nielbock, M., Reipurth, B., \& Sievers, A. 2001, A\&A, 369. 155

Clarke, C. J., Gendrin, A., \& Sotomayor, M. 2001, MNRAS, 328, 485

Cohen, M., \& Kuhi, L. V. 1979, ApJS, 41, 743

Cohen, J. G., Persson, S. E., Elias, J. H., \& Frogel, J. A. 1981, ApJ, 249, 481

Corder, S., Eisner, J., \& Sargent, A. 2005, ApJ, 622, L133

D'Antona, F., \& Mazzitelli, I. 1997, in Cool Stars in Clusters and Associations, eds. G. Micela \& R. Pallavicini (Firenze: Soc. Astron. Italiana), 807

Dartois, E., Dutrey, A., \& Guilloteau, S. 2003, A\&A, 399, 773

Dent, W. R. F., Matthews, H. E., \& Ward-Thompson, D. 1998, MNRAS, 301, 1049

Dowell, C. D. et al. 2003, Proc. SPIE, 4855, 73

Draine, B. T., \& Lee, H. M. 1984, ApJ, 285, 89

Duchêne, G., Monin, J.-L., Bouvier, J., \& Ménard, F. 1999, A\&A, 351, 954

Duchêne, G., Bouvier, J., Bontemps, S., André, P., \& Motte, F. 2004, A\&A, 427, 651 (a)

Duchêne, G., McCabe, C., Ghez, A. M., \& Macintosh, B. A. 2004, ApJ, 606, 969

Dullemond, C. P., Dominik, C., \& Natta, A. 2001, ApJ, 560, 957

Dullemond, C. P., \& Dominik, C. 2005, A\&A, 434, 971

Dutrey, A., Guilloteau, S., \& Simon, M. 1994, A\&A, 286, 149

Dutrey, A., Guilloteau, S., Duvert, G., Prato, L., Simon, M., Schuster, K., \& Ménard, F. 1996, A\&A, 309, 493

Dutrey, A., Guilloteau, S., \& Simon, M. 2003, A\&A, 401, 1003 
Duvert, G., Dutrey, A., Guilloteau, S., Ménard, F., Schuster, K., Prato, L., \& Simon, M. 1998, A\&A, 332, 867

Duvert, G., Guilloteau, S., Ménard, F., Simon, M., \& Dutrey, A. 2000, A\&A, 355, 165

Elias, J. H. 1978, ApJ, 224, 857

Erickson, E. F., Knacke, R. F., Tokunaga, A. T., \& Haas, M. R. 1981, ApJ, 245, 148

Feigelson, E. D., \& Nelson, P. I. 1985, ApJ, 293, 192

Fischer, D. A., Marcy, G. W., Butler, R. P., Vogt, S. S., Frink, S., \& Apps, K. 2001, ApJ, 551, 1107

Forrest, W. J., et al. 2004, ApJS, 154, 443

Ghez, A. M., Neugebauer, G., \& Matthews, K. 1993, AJ, 106, 2005

Gomez, M., Jones, B. F., Hartmann, L., Kenyon, S. J., Stauffer, J. R., Hewett, R., \& Reid, I. N. 1992, AJ, 104, 762

Greene, T. P., Wilking, B. A., André, P., Young, E. T., \& Lada, C. J. 1994, ApJ, 434, 614

Guilloteau, S., \& Dutrey, A. 1998, A\&A, 339, 467

Haas, M., Leinert, C., \& Zinnecker, H. 1990, A\&A, 230, L1

Haisch, K. E., Lada, E. A., \& Lada, C. J. 2001, ApJ, 553, L153

Hartigan, P., Strom, K. M., \& Strom, S. E. 1994, ApJ, 427, 961

Hartmann, L., Jones, B. F., Stauffer, J. R., \& Kenyon, S. J. 1991, AJ, 101, 1050

Hartmann, L., Hewett, R., \& Calvet, N. 1994, ApJ, 426, 669

Hartmann, L., Calvet, N., Gullbring, E., \& D’Alessio, P. 1998, ApJ, 495, 385

Hartmann, L., Megeath, S. T., Allen, L., Luhman, K., Calvet, C., D’Alessio, P., Franco-Hernandez, R., \& Fazio, G. 2005, ApJ, in press (astro-ph/0505323)

Henning, T., Michel, B., \& Stognienko, R. 1995, Plan. Space. Sci., 43, 1333

Henning, T., \& Stognienko, R. 1996, A\&A, 311, 291

Herbig, G. H., Vrba, F. J., \& Rydgren, A. E. 1986, AJ, 91, 575

Herbig, G. H., \& Bell, K. R. 1988, Lick Obs. Bull. 1111

Hernández, J., Calvet, N., Briceño, C., Hartmann, L., \& Berlind, P. 2004, AJ, 127, 1682 
Hildebrand, R. 1983, QJRAS, 24, 267

Hogerheijde, M. R., \& Sandell, G. 2000, ApJ, 534, 880

Hogerheijde, M. R., Johnstone, D., Matsuyama, I., Jayawardhana, R., \& Muzerolle, J. 2003, ApJ, 593, L101

Holland, W. S. et al. 1999, MNRAS, 303, 659

Hollenbach, D. J., Yorke, H. W., \& Johnstone, D. 2000, in Protostars \& Planets IV, eds. V. Mannings, A. P. Boss, \& S. S. Russell (Tucson: Univ. Arizona Press), 401

Jenness, T., \& Lightfoot, J. F. 1998, in ASP Conf. Proc. 145, Astronomical Data Analysis Software and Systems VII, ed. R. Albrecht, R. N. Hook, \& H. A. Bushouse (San Francisco: ASP), 216

Jenness, T., Lightfoot, J. F., \& Holland, W. S. 1998, Proc. SPIE, 3357, 548

Jenness, T., Stevens, J. A., Archibald, E. N., Economous, F., Jessop, N. E., \& Robson, E. I. 2002, MNRAS, 336, 14

Jensen, E. L. N., \& Akeson, R. L. 2003, ApJ, 584, 875

Jensen, E. L. N., Mathieu, R. D., \& Fuller, G. A. 1994, ApJ, 429, L29

Jensen, E. L. N., Koerner, D. W., \& Mathieu, R. D. 1996, AJ, 111, 2431

Jensen, E. L. N., Mathieu, R. D., \& Fuller, G. A. 1996, ApJ, 458, 312

Johns-Krull, C. M., \& Valenti, J. A. 2001, ApJ, 561, 1060

Johnstone, D., Hollenbach, D., \& Bally, J. 1998, ApJ, 499, 758

Jones, B. F., \& Herbig, G. H. 1979, AJ, 84, 1872

Kamp, I., \& Dullemond, C. P. 2004, 615, 991

Kenyon, S. J., \& Hartmann, L. 1987, ApJ, 323, 714

Kenyon, S. J., \& Hartmann, L. 1995, ApJS, 101, 117

Kessler-Silacci, J. E., Hillenbrand, L. A., Blake, G. A., \& Meyer, M. R. 2005, ApJ, 622, 404

Kitamura, Y., Momose, M., Yokogawa, S., Kawabe, R., Tamura, M., \& Ida, S. 2002, ApJ, 581, 357

Koerner, D. W., Sargent, A. I., \& Beckwith, S. V. W. 1993, ApJ, 408, L93 (a)

Koerner, D. W., Sargent, A. I., \& Beckwith, S. V. W. 1993, Icarus, 106, 2 (b)

Koerner, D. W., \& Sargent, A. I. 1995, AJ, 109, 2138 
Koerner, D. W., Chandler, C. J., \& Sargent, A. I. 1995, ApJ, 452, L69

Kovács, A. et al., in preparation

Kroupa, P. 1995, MNRAS, 277, 1522

Lada, C. J., \& Wilking, B. A. 1984, ApJ, 287, 610

Laughlin, G., \& Bodenheimer, P. 1994, ApJ, 436, 335

LaValley, M., Isobe, T., \& Feigelson, E. D. 1990, BAAS, 22, 917

Leinert, C., \& Haas, M. 1989, ApJ, 342, L39

Leinert, C., Haas, M., Mundt, R., Richichi, A., \& Zinnecker, H. 1991, A\&A, 250, 407

Leinert, C., Zinnecker, H., Weitzel, N., Christou, J., Ridgway, S. T., Jameson, R., Haas, M., \& Lenzen, R. 1993, A\&A, 278, 129

Leinert, C., Richichi, A., \& Haas, M. 1997, A\&A, 318, 472

Mannings, V., \& Emerson, J. P. 1994, MNRAS, 267, 361

Mannings, V. 1994, MNRAS, 271, 587

Mannings, V., \& Sargent, A. I. 1997, ApJ, 490, 792

Mannings, V., \& Sargent, A. I. 2000, ApJ, 529, 391

Marcy, G. W., Butler, R. P., Vogt, S. S., Fischer, D. A., Henry, G. W., Laughlin, G., Wright, J. T., \& Johnson, J. A. 2005, ApJ, 619, 570

Martín, E. L., Rebolo, R., Magazzú, A., \& Pavlenko, Y. V. 1994, A\&A, 282, 503

Martín, E. L., Magazzú, A., Delfosse, X., \& Mathieu, R. D. 2005, A\&A, 429, 939

Mathieu, R. D. 1994, ARA\&A, 32, 465

Mathieu, R. D., Adams, F. C., Fuller, G. A., Jensen, E. L. N., Koerner, D. W., \& Sargent, A. I. 1995, AJ, 109, 2655

Mathieu, R. D., Stassun, K., Basri, G., Jensen, E. L. N., Johns-Krull, C. M., Valenti, J. A., \& Hartmann, L. W. 1997, AJ, 113, 1841

Mathieu, R. D., Ghez, A. M., Jensen, E. L. N., \& Simon, M. 2000, in Protostars \& Planets IV, eds. V. Mannings, A. P. Boss, \& S. S. Russell (Tucson: Univ. Arizona Press), 703

McCabe, C., Duchêne, G., \& Ghez, A. M. 2003, ApJ, 588, L113

Meeus, G., Sterzik, M., Bouwman, J., \& Natta, A. 2003, A\&A, 409, L25 
Men'shchikov, A. B., Henning, T., \& Fischer, O. 1999, ApJ, 519, 257

Meyer, M. R., Calvet, N., \& Hillenbrand, L. A. 1997, AJ, 114, 288

Miyake, K., \& Nakagawa, Y. K. 1993, Icarus, 106, 20

Mizuno, H. 1980, Journal of Progressive Theoretical Physics, 64, 544

Motte, F., \& André, P. 2001, A\&A, 365, 440

Mundt, R., Walter, F. M., Feigelson, E. D., Finkenzeller, U., Herbig, G. H., \& Odell, A. P. 1983, ApJ, 269, 229

Muzerolle, J., Hartmann, L., \& Calvet, N. 1998, AJ, 116, 455

Muzerolle, J., Calvet, N., \& Hartmann, L. 2001, ApJ, 550, 944

Muzerolle, J., Calvet, N., Hartmann, L., \& D’Alessio, P. 2003, ApJ, 597, L149

Najita, J. R., Edwards, S., Basri, G., \& Carr, J. 2000, in Protostars \& Planets IV, eds. V. Mannings, A. P. Boss, \& S. S. Russell (Tucson: Univ. Arizona Press), 559

Natta, A., Grinin, V. P., \& Mannings, V. 2001, in Protostars \& Planets IV, eds. V. Mannings, A. P. Boss, \& S. S. Russell (Tucson: Univ. Arizona Press), 559

Natta, A., Testi, L., Neri, R., Shepherd, D. S., \& Wilner, D. J. 2004, A\&A, 416, 179

O’Dell, C. R., \& Wen, Z. 1994, ApJ, 436, 194

Osterloh, M., \& Beckwith, S. V. W. 1995, ApJ, 439, 288

Pollack, J. B., Hollenbach, D., Beckwith, S., Simonelli, D. P., Roush, T., \& Fong, W. 1994, ApJ, 421,615

Pollack, J. B., Hubickyj, O., Bodenheimer, P., Lissauer, J. J., Podolak, M., \& Greenzweig, Y. 1996, Icarus, 124,62

Przygodda, F., van Boekel, R., Àbrahàm, P., Melnikov, S. Y., Waters, L. B. F. M., \& Leinert, C. 2003, A\&A, 412, L43

Qi, C., Kessler, J. E., Koerner, D. W., Sargent, A. I., \& Blake, G. A. 2003, ApJ, 597, 986

Reid, N., Hawley, S. L., \& Mateo, M. 1995, MNRAS, 272, 828

Reipurth, B., \& Zinnecker, H. 1993, A\&A, 278, 81

Richichi, A., Leinert, C., Jameson, R., \& Zinnecker, H. 1994, A\&A, 287, 145

Rodríguez, L. F., Cantó, J., Torrelles, J. M., \& Ho, P. T. P. 1986, ApJ, 301, L25 
Sandell, G., \& Weintraub, D. A. 2001, ApJS, 134, 115

Schwartz, P. R. 1982, ApJ, 252, 589

Semenov, D., Pavlyuchenkov, Y., Schreyer, K., Henning, T., Dullemond, C., \& Bacmann, A. 2005, ApJ, 621, 853

Shirley, Y. L., Evans, N. J., Rawlings, J. M. C., \& Gregersen, E. M. 2000, ApJS, 131, 249

Shu, F. H., Tremaine, S., Adams, F. C., \& Ruden, S. P. 1990, ApJ, 358, 495

Simon, M., Howell, R. R., Longmore, A. J., Wilking, B. A., Peterson, D. M., \& Chen, W.-P. 1987, ApJ, 320, 344

Simon, M., Chen, W.-P., Howell, R. R., Benson, J. A., \& Slowick, D. 1992, ApJ, 384, 212

Simon, M., Ghez, A. M., Leinert, C., Cassar, L., Chen, W.-P., Howell, R. R., Jameson, R. F., Matthews, K., Neugebauer, G., \& Richichi, A. 1995, ApJ, 443, 625

Simon, M., Dutrey, A., \& Guilloteau, S. 2001, ApJ, 545, 1035

Skrutskie, M. F., Dutkevitch, D., Strom, S. E., Edwards, S., Strom, K. M., \& Shure, M. A. 1990, AJ, 99, 1187

Slutskiı̌, V. E., Stal'bovskiǔ, O. I., \& Shevchenko, V. S. 1980, Sov. Astron. Lett., 6, 397

Stapelfeldt, K. R., Ménard, F., Watson, A. M., Krist, J. E., Dougados, C., Padgett, D. L., \& Brandner, W. 2003, ApJ, 589, 410

Stelzer, B., \& Neuhäuser, R. 2001, A\&A, 377, 538

Strom, K. M., Strom, S. E., Edwards, S., Cabrit, S., \& Skrutskie, M. F. 1989, AJ, 97, 1451

Tamazian, V. S. 2004, AJ, 127, 2378

Terebey, S., Shu, F. H., \& Cassen, P. 1984, ApJ, 286, 529

Testi, L., Natta, A., Shepherd, D. S., \& Wilner, D. J. 2001, ApJ, 554, 1087

Testi, L., Natta, A., Shepherd, D. S., \& Wilner, D. J. 2003, A\&A, 403, 323

van Boekel, R., et al. 2004, Nature, 432, 479

van Zadelhoff, G.-J., van Dishoeck, E. F., Thi, W.-F., \& Blake, G. A. 2001, A\&A, 377, 566

van Zadelhoff, G.-J., Aikawa, Y., Hogerheijde, M. R., \& van Dishoeck, E. F. 2003, A\&A, 397, 789

Weaver, W. B., \& Jones, G. 1992, ApJS, 78, 239

Weintraub, D. A., Sandell, G., \& Duncan, W. D. 1989, ApJ, 340, L69 
Weintraub, D. A., Masson, C. R., \& Zuckerman, B. 1989, ApJ, 344, 915

Weintraub, D. A., Sandell, G., Huard, T. L., Kastner, J. H., van den Ancker, M. E., \& Waters, R. 1999, ApJ, 517, 819

Weidenschilling, S. J. 1977, Ap\&SS, 51, 153

Weidenschilling, S. J., \& Cuzzi, J. N. 1993, in Protostars \& Planets III, eds. E. H. Levy \& J. I. Lunine (Tucson: Univ. Arizona Press), 1031

White, R. J., \& Ghez, A. M. 2001, ApJ, 556, 265

White, R. J., \& Hillenbrand, L. A. 2004, ApJ, 616, 998

White, R. J., \& Hillenbrand, L. A. 2005, ApJ, 621, L65

Wolk, S. J., \& Walter, F. M. 1996, AJ, 111, 2066

Wright, E. L. 1987, ApJ, 320, 818

Young, C. H., Shirley, Y. L., Evans, N. J., \& Rawlings, J. M. C. 2003, ApJS, 145, 111 

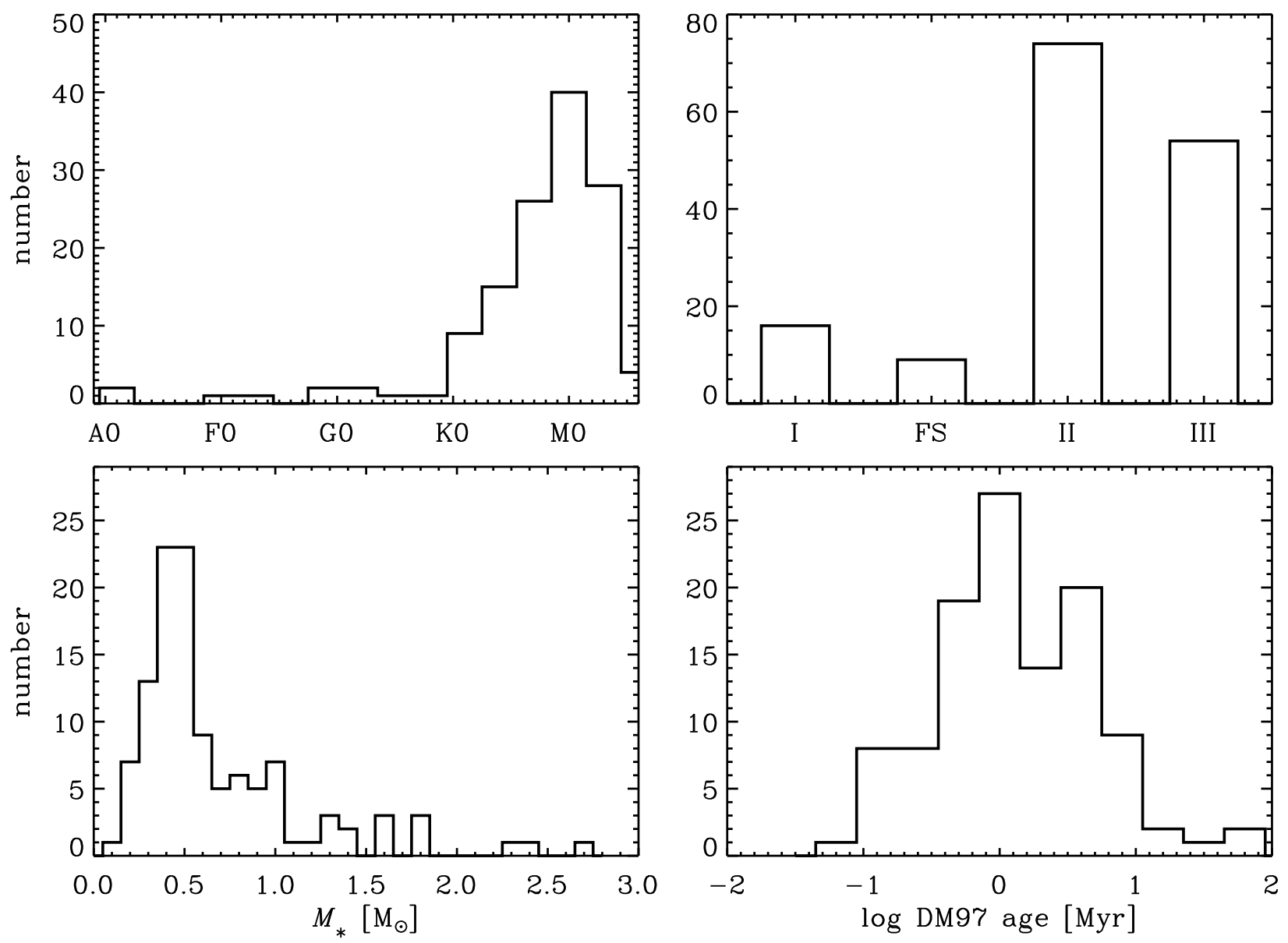

Fig. 1.- Histograms highlighting some key properties of the submillimeter sample. The upper left panel shows the distribution of spectral types from the literature (see Table 1), which is primarily constrained to $\mathrm{K}$ and $\mathrm{M}$ types. The upper right panel marks the relative numbers of YSOs of various SED classifications (Class I, Flat-Spectrum, Class II, and Class III; see also Table 1): these classifications are discussed in detail in $\S 4$. The bottom panels show the number distributions of stellar masses and ages, inferred as described in $§ 3.4$ using the D’Antona \& Mazzitelli (1997) theoretical pre-main-sequence models. 


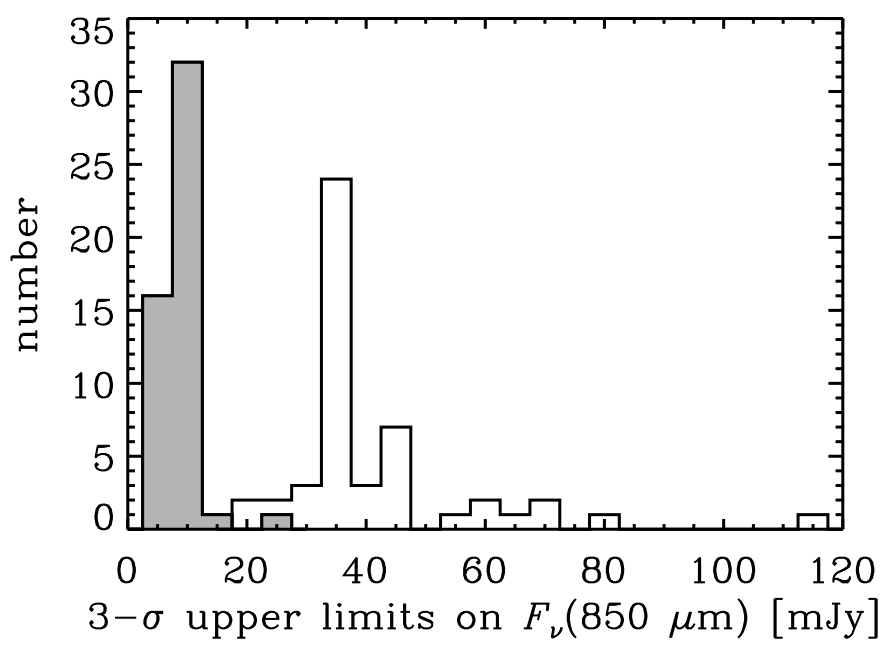

Fig. 2.- The distributions of 3- $\sigma$ upper limits for the sources which were not detected in both this survey (filled histogram) and the combined surveys of Beckwith et al. (1990) and Osterloh \& Beckwith (1995) at $1.3 \mathrm{~mm}$ (unfilled histogram). The upper limits are in units of mJy, and the $1.3 \mathrm{~mm}$ measurements have been scaled according to $F_{\nu} \propto \nu^{2}$ (see $\S 3.3$ ) to enable direct comparison with the $850 \mu \mathrm{m}$ measurements. The submillimeter survey presented here is roughly a factor of 5 more sensitive in terms of flux density limits, and is considerably more uniform. 

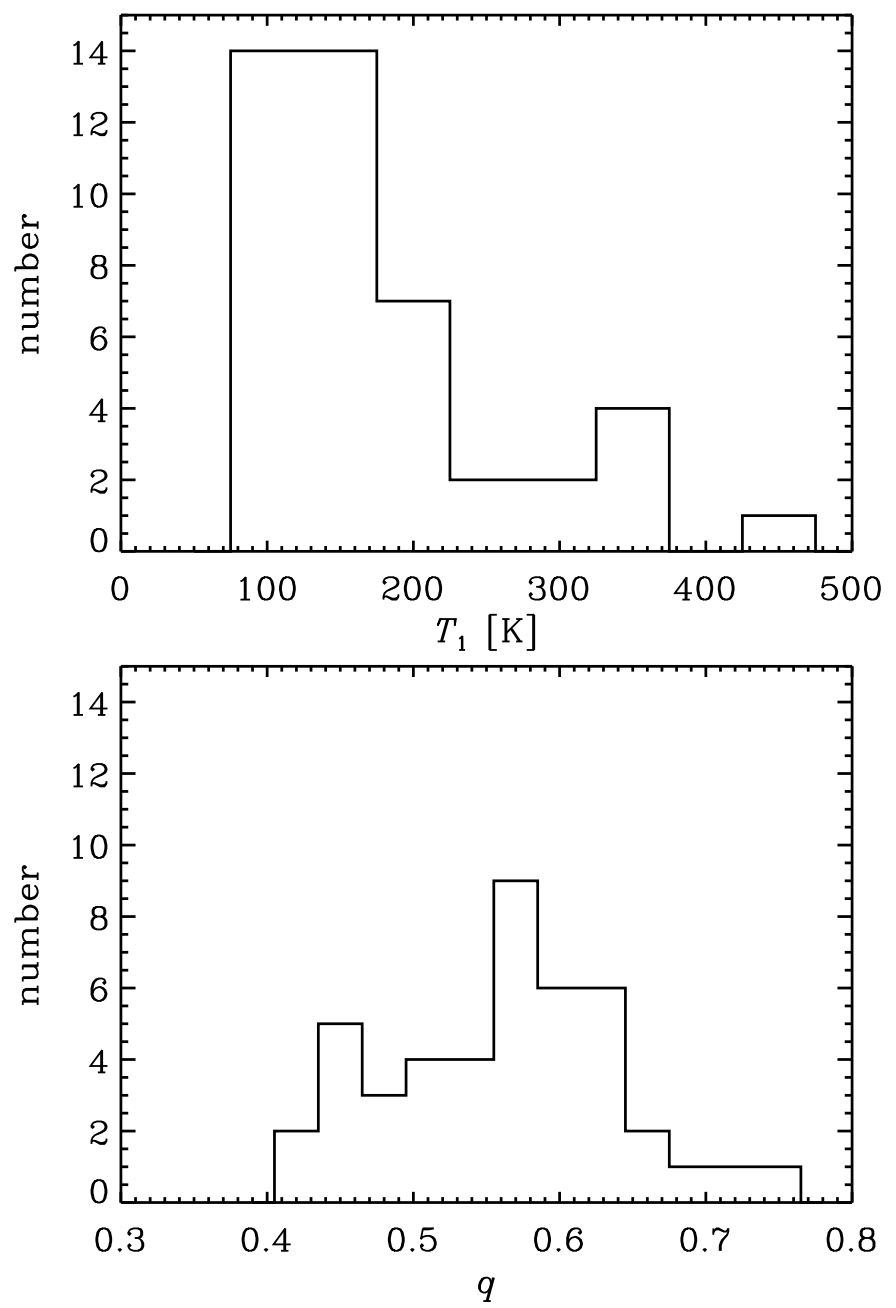

Fig. 3.- The distributions of the best-fit disk model parameters of the normalization ( $T_{1}$ : top $)$ and power law index $(q$ : bottom) of the radial temperature profile. The mean and median values for the sample are $0.56 \pm 0.08$ and 0.58 for $q$, and $178 \pm 85 \mathrm{~K}$ and $148 \mathrm{~K}$ for $T_{1}$ (quoted errors are standard deviations). 


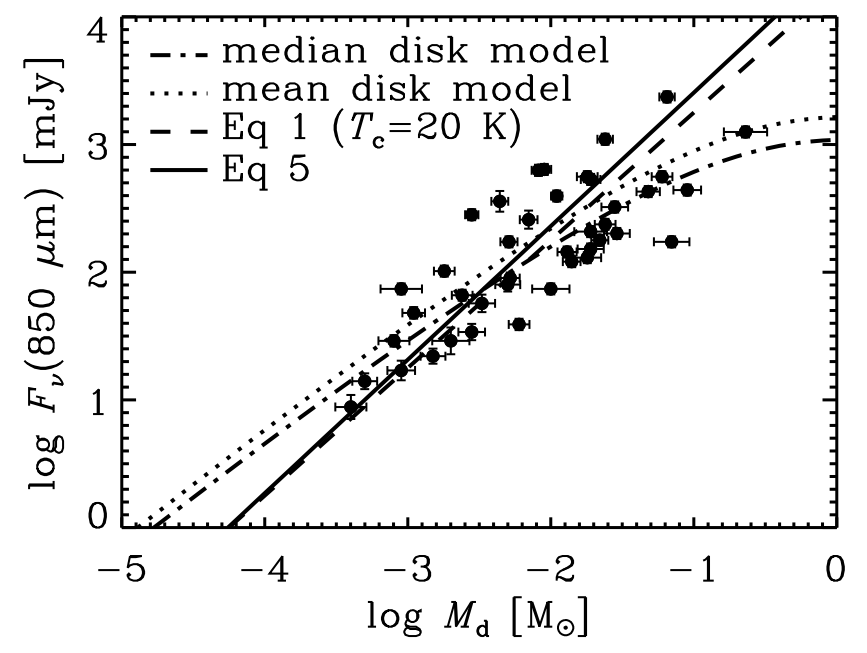

Fig. 4.- The relationship between the disk mass and $850 \mu \mathrm{m}$ flux density for the sources in Table 2. The solid line is the best linear fit to the data in log-log space, given as Equation 5 in the text. The dashed line is the best fit to the data based on the optically thin, isothermal disk model given in Equation 1: the best-fit characteristic temperature is $T_{c}=20 \mathrm{~K}$. The dotted and dash-dotted curves show the mean and median disk model behaviors, as described in the text. Although a considerable dispersion exists (the rms residual dispersion around the best-fit power law model given in Equation 5 is $0.2 \mathrm{dex}$ ), the apparent correlation here permits an empirical calibration of the $M_{d}-F_{\nu}$ relationship. 

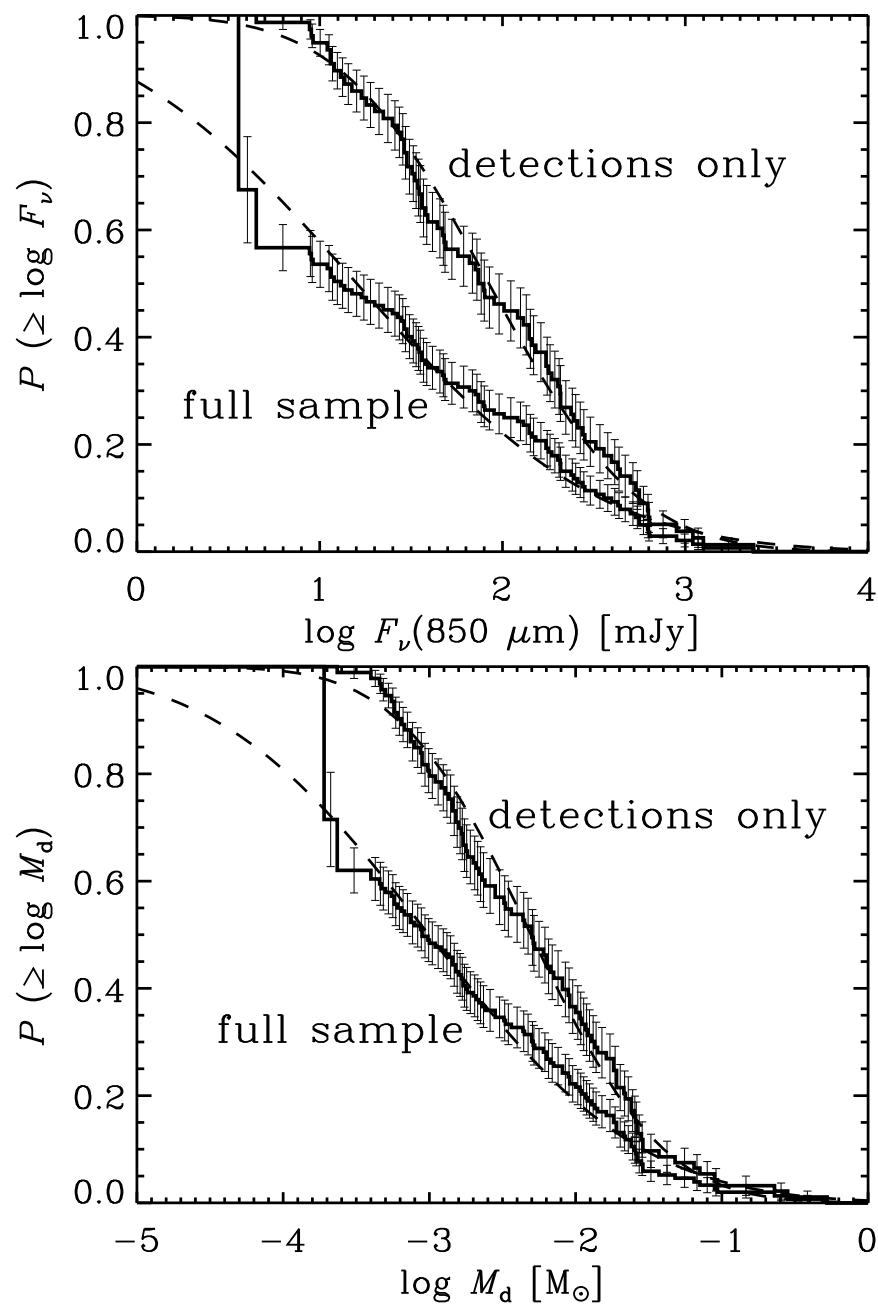

Fig. 5.- Cumulative distributions of $850 \mu \mathrm{m}$ flux densities for 140 objects in the full sample and the 78 of those which have $\geq 3-\sigma$ detections (top) and disk masses for 153 objects in the full sample and 93 objects which have submillimeter detections (bottom). The full sample distribution functions were computed using the Kaplan-Meier estimator to incorporate 3- $\sigma$ upper limits. The ordinate values represent the probability that an object in the sample has a flux density or disk mass greater than or equal to each abscissa value. The flux densities are log-normally distributed: the full sample with mean $1.20 \pm 0.02(16 \mathrm{mJy})$ and variance $1.08 \pm 0.06 \mathrm{dex}$ and the detections subsample with mean $1.93 \pm 0.01$ ( $85 \mathrm{mJy}$ ) and variance $0.41 \pm 0.02$ dex. The same distribution holds for the disk masses: the full sample with mean $-3.00 \pm 0.02\left(10^{-3} \mathrm{M}_{\odot}\right)$ and variance $1.31 \pm 0.06 \mathrm{dex}$ and the detections subsample with mean $-2.31 \pm 0.01\left(5 \times 10^{-3} \mathrm{M}_{\odot}\right)$ and variance $0.50 \pm 0.02$ dex. The best-fit distributions are overlaid as dashed curves. 

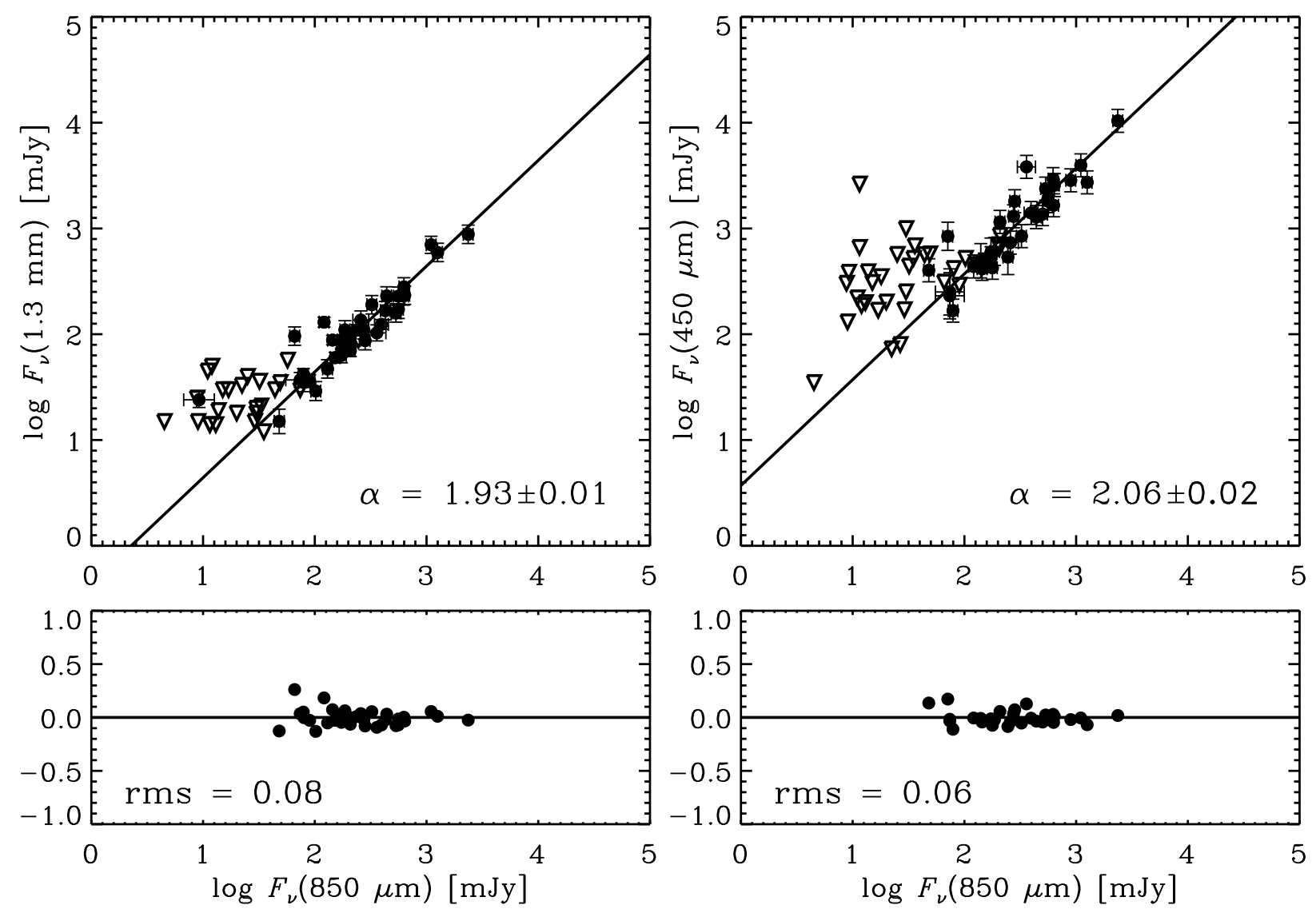

Fig. 6. - The upper panels show $1.3 \mathrm{~mm}$ (left) and $450 \mu \mathrm{m}$ (right) flux densities plotted against $850 \mu \mathrm{m}$ flux densities. The open triangles mark 3- $\sigma$ upper limits. For sources with no obvious error bars, errors are smaller than the symbol size. The solid lines are power laws of the form $F_{\nu} \propto \nu^{\alpha}$, and the best-fit value of $\alpha$ is given in the lower right corner (determined from the intercept of a linear fit in $\log$-log space). The fits are only conducted for $\log F_{\nu}(850 \mu \mathrm{m})>1.5$, and sources with anomalous continuum slopes are excluded (see Table 1). The bottom panels give the residuals to the fits and the rms values of the residuals. Considering the scatter around the best-fit relationships, the data indicate that the continuum slope is roughly the same regardless of the wavelength range in which it is measured. 


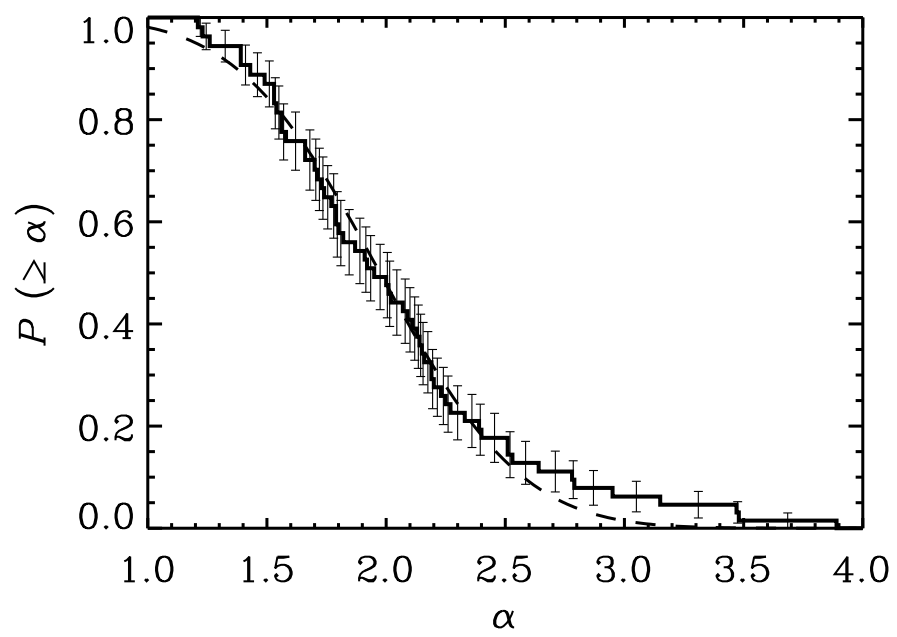

Fig. 7.- The cumulative distribution of submillimeter continuum slopes ( $\alpha$ : defined by $\left.F_{\nu} \propto \nu^{\alpha}\right)$ for 84 YSOs, computed using the Kaplan-Meier estimator to incorporate 3- $\sigma$ upper limits. The ordinate values represent the probability that an object in the sample has a continuum slope greater than or equal to $\alpha$. The continuum slopes are normally distributed with mean $1.97 \pm 0.01$ and variance $0.22 \pm 0.02$, overlaid with a dashed curve. The high slope tail of the distribution is slightly enhanced. 


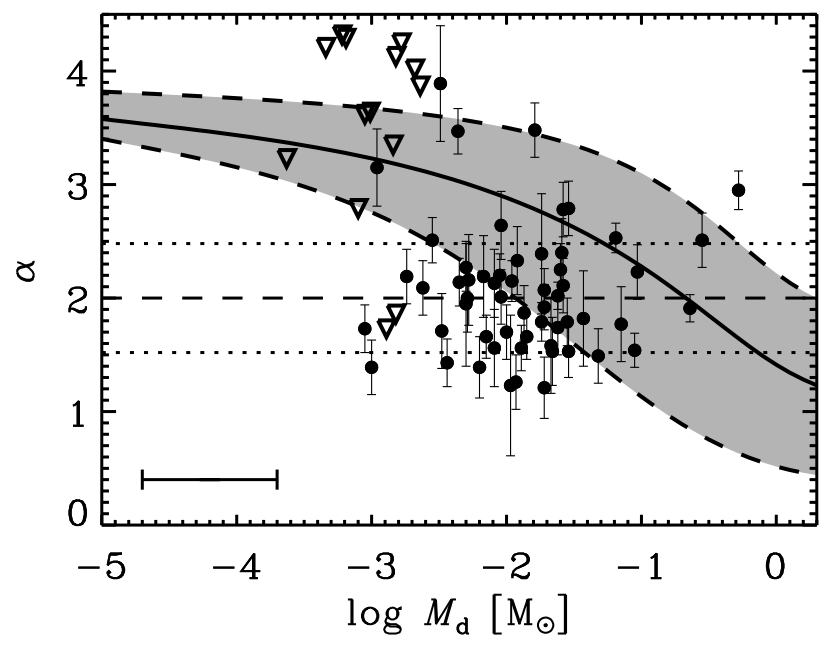

Fig. 8. - The submillimeter continuum slope values ( $\alpha$ : defined as $\left.F_{\nu} \propto \nu^{\alpha}\right)$ plotted against the logarithm of the disk mass. The open triangles are 3- $\sigma$ upper limits on $\alpha$. A typical error bar on the disk mass which incorporates the systematic uncertainties $(\sim 0.5 \mathrm{dex})$ due to the unknown value of the opacity index is shown in the lower left corner. The dashed horizontal line marks the best-fit mean value of $\alpha$, determined from the cumulative distribution shown in Figure 7. The dotted lines parallel to it mark the 1- $\sigma$ standard deviation from the mean. The thick, solid curve marks the behavior of $\alpha\left(M_{d}\right)$ for the median disk model with $\beta=2$. The shaded region marks the $\beta=2$ curves for extreme temperature profiles: the lower boundary for $T_{1}=75 \mathrm{~K}$ and $q=0.75$ and the upper boundary for $T_{1}=300 \mathrm{~K}$ and $q=0.35$. Much of the sample appears to have $\beta<2$, indicating a change in the opacity function in disk material relative to the interstellar medium which could be a signature of the collisional growth of dust grains. 

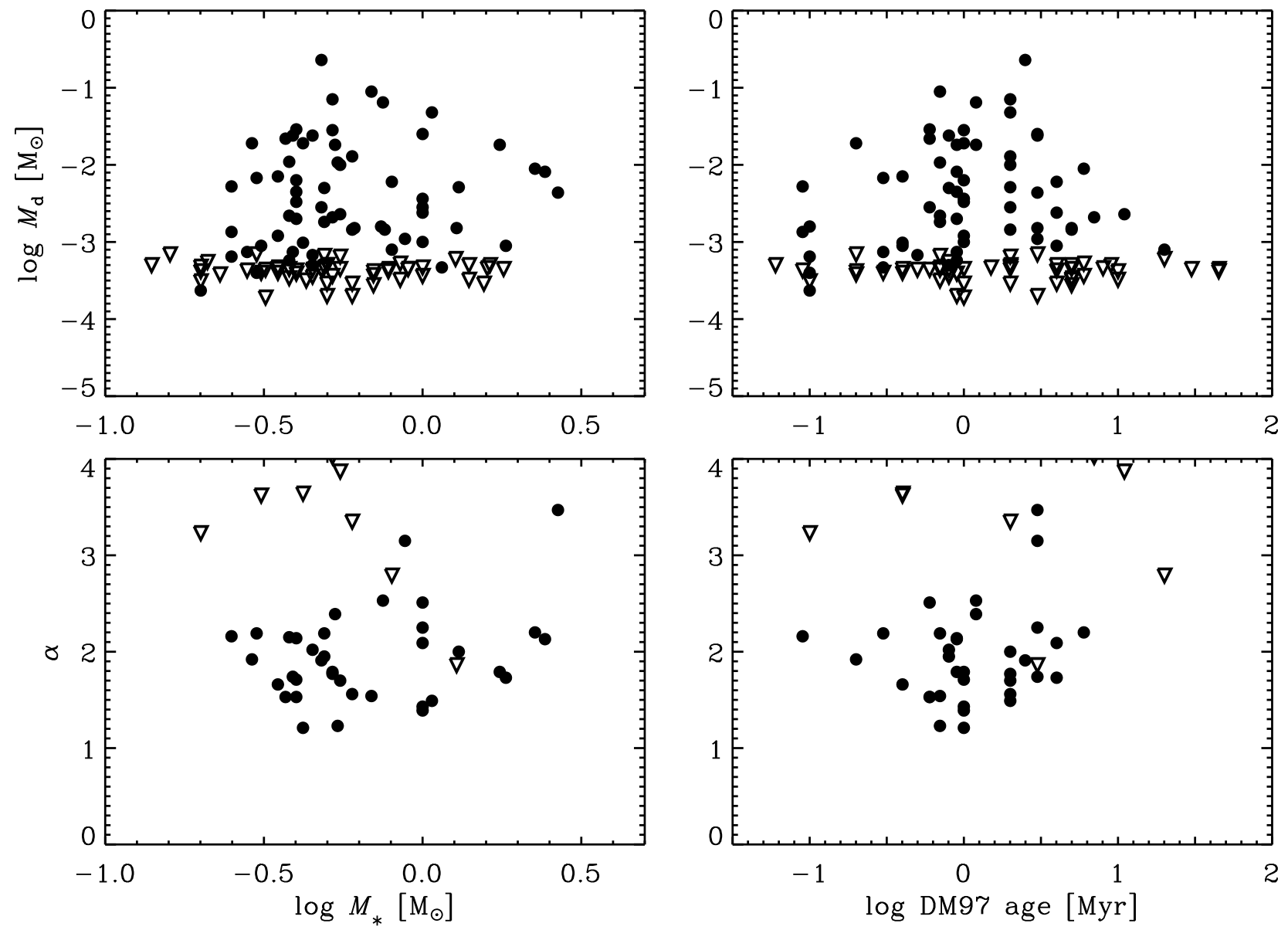

Fig. 9.- Plots showing the relationships between disk masses or submillimeter continuum slopes and stellar masses or ages. Filled circles are detections and open triangles are 3- $\sigma$ upper limits. 


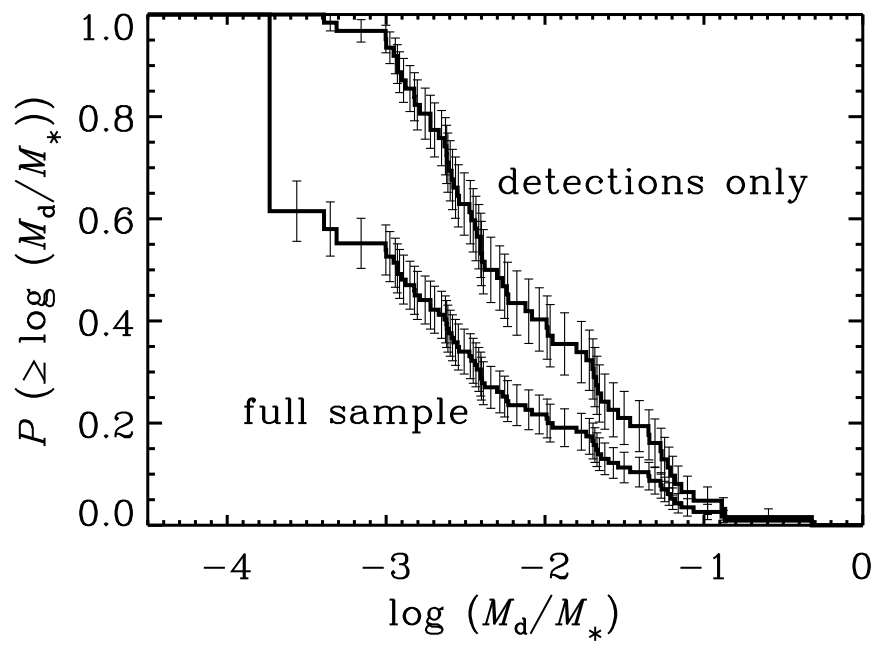

Fig. 10. - The cumulative distributions of the disk to star mass ratio for 116 objects in the full sample (constructed with the Kaplan-Meier estimator) and 61 objects which were detected at submillimeter wavelengths. The ordinate values represent the probability that an object in the sample has a mass ratio greater than or equal to the abscissa value. Less than $10 \%$ of the sample has a mass ratio which could result in a gravitational disk instability, and even this small fraction may be contaminated by mass in an envelope. The median disk to star mass ratio is $0.5 \%$. 


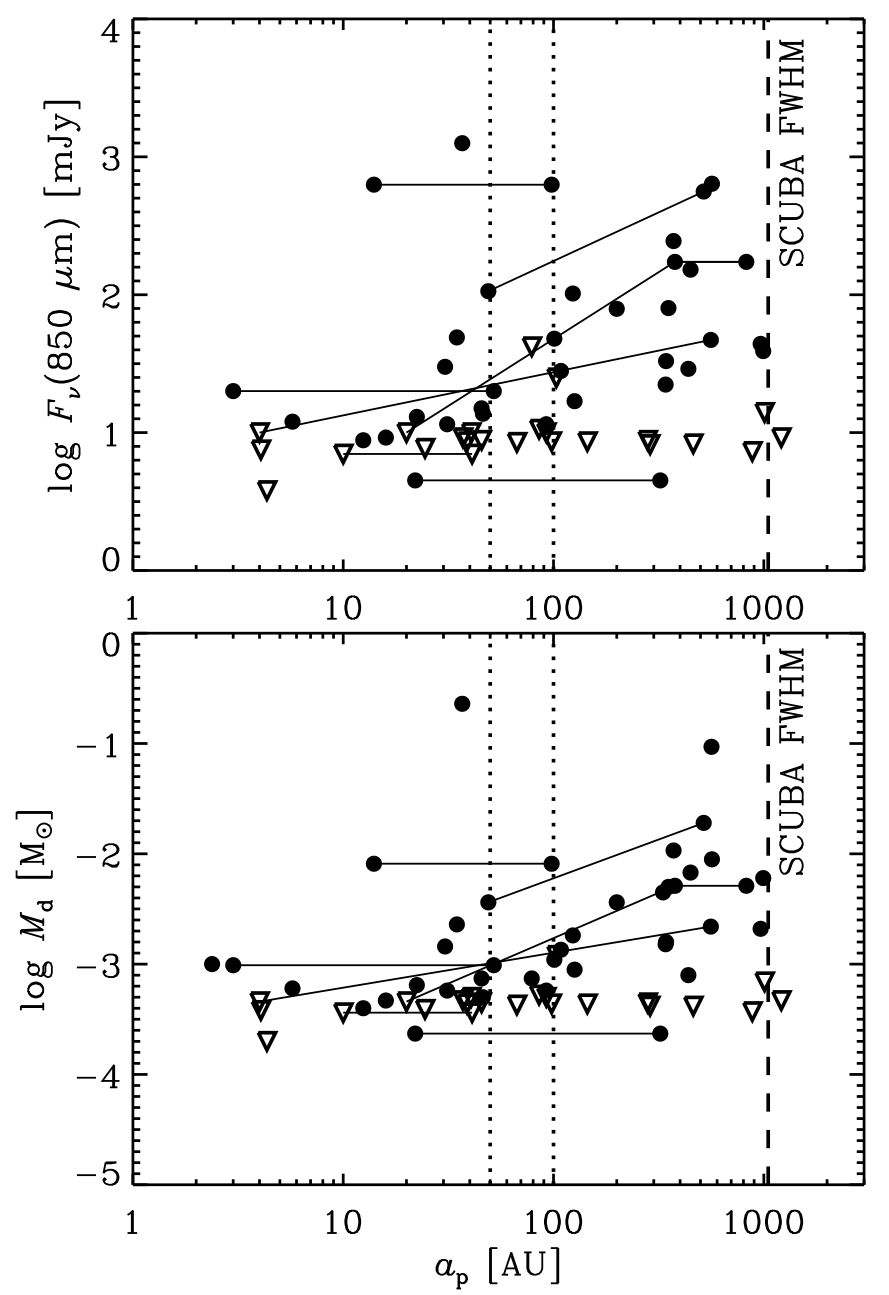

Fig. 11. - The relationship between the $850 \mu \mathrm{m}$ flux density (top) or disk mass (bottom) and projected semimajor axis for multiple star systems which were not resolved by the observations. Open triangles are 3- $\sigma$ upper limits, and higher order systems are connected with solid lines. The dashed vertical lines mark the FWHM beam radius of SCUBA at $850 \mu \mathrm{m}$, while the dotted lines mark 50 and $100 \mathrm{AU}$. For Class II multiple systems, the flux densities and disk masses in close binary systems $\left(a_{p} \leq 50\right.$ or $\left.100 \mathrm{AU}\right)$ are statistically lower than those in wider binary systems or single stars (see Table 4). 

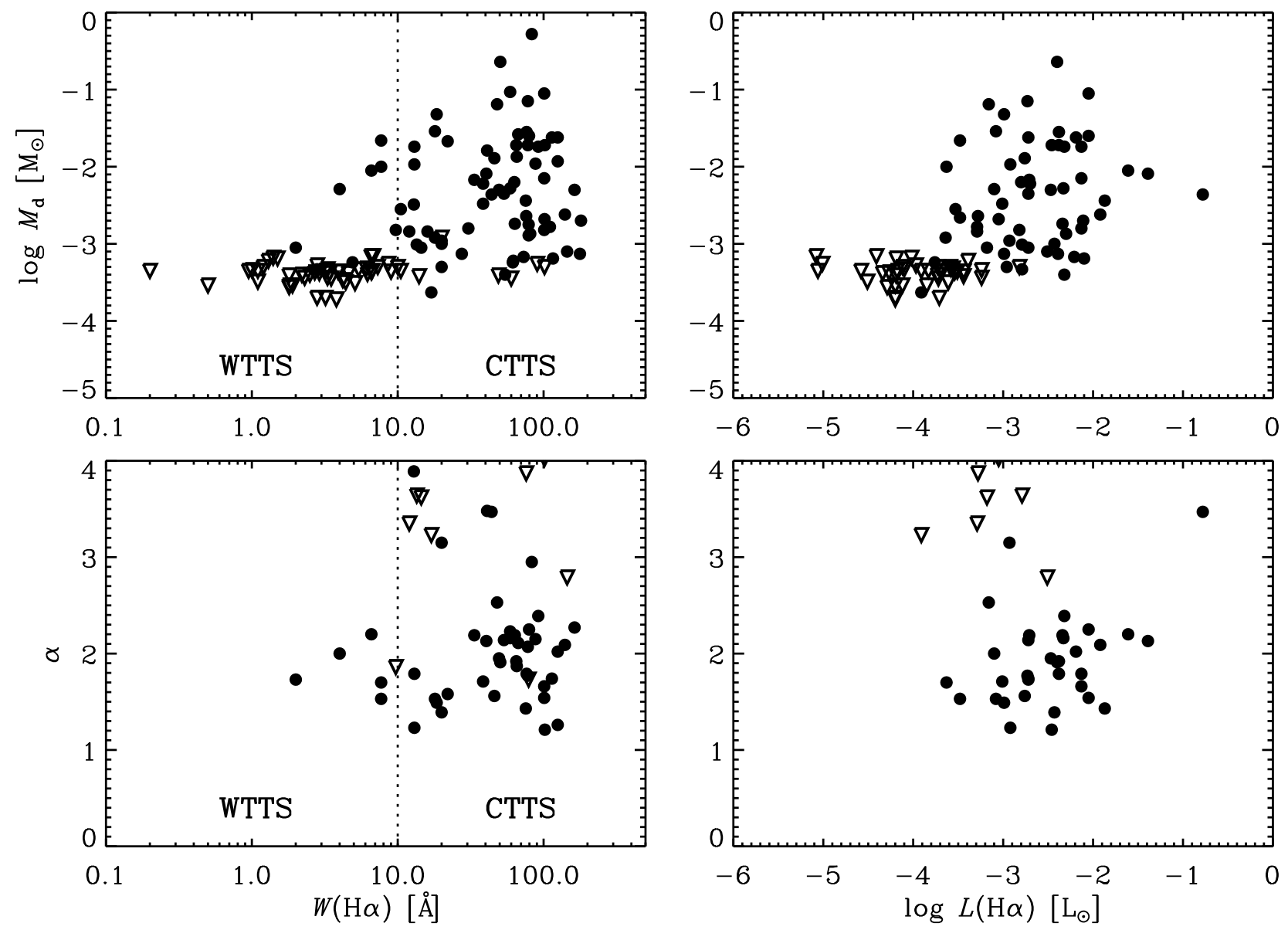

Fig. 12.- The behavior of disk mass or submillimeter continuum slope for various ranges of the equivalent width or luminosity of the $\mathrm{H} \alpha$ emission line. Filled circles are detections and open triangles are 3- $\sigma$ upper limits. The dotted vertical lines mark the boundary between WTTSs and CTTSs. Luminosities in the $\mathrm{H} \alpha$ line were computed from equivalent widths and a linear relationship between the local continuum flux and the extinction-corrected $R$-band flux (Reid, Hawley, \& Mateo 1995, see their Figure 1a). 

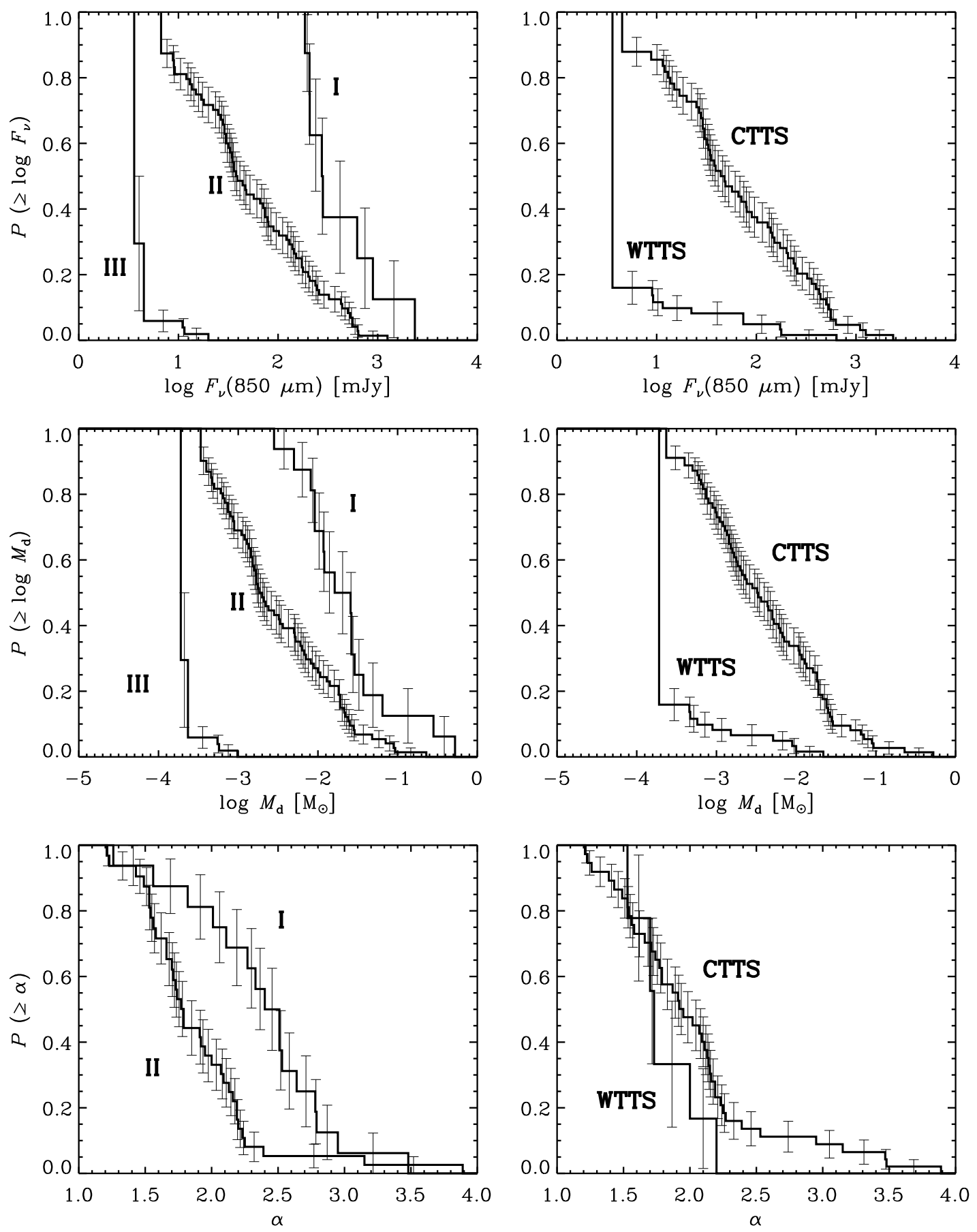

Fig. 13.- Evidence for outer disk evolution from the distribution functions of the $850 \mu \mathrm{m}$ flux densities (top), circumstellar disk masses (middle) and submillimeter continuum slopes (bottom). Each cumulative distribution is labeled with the infrared SED or $W(\mathrm{H} \alpha)$ classification from which it was constructed. Statistical tests (see Table 6) confirm that there is a decrease in $F_{\nu}$ and $M_{d}$ from Class I $\rightarrow$ II $\rightarrow$ III and CTTS $\rightarrow$ WTTS sources, and that $\alpha$ is also diminished in the evolution from Class I $\rightarrow$ II sources. 

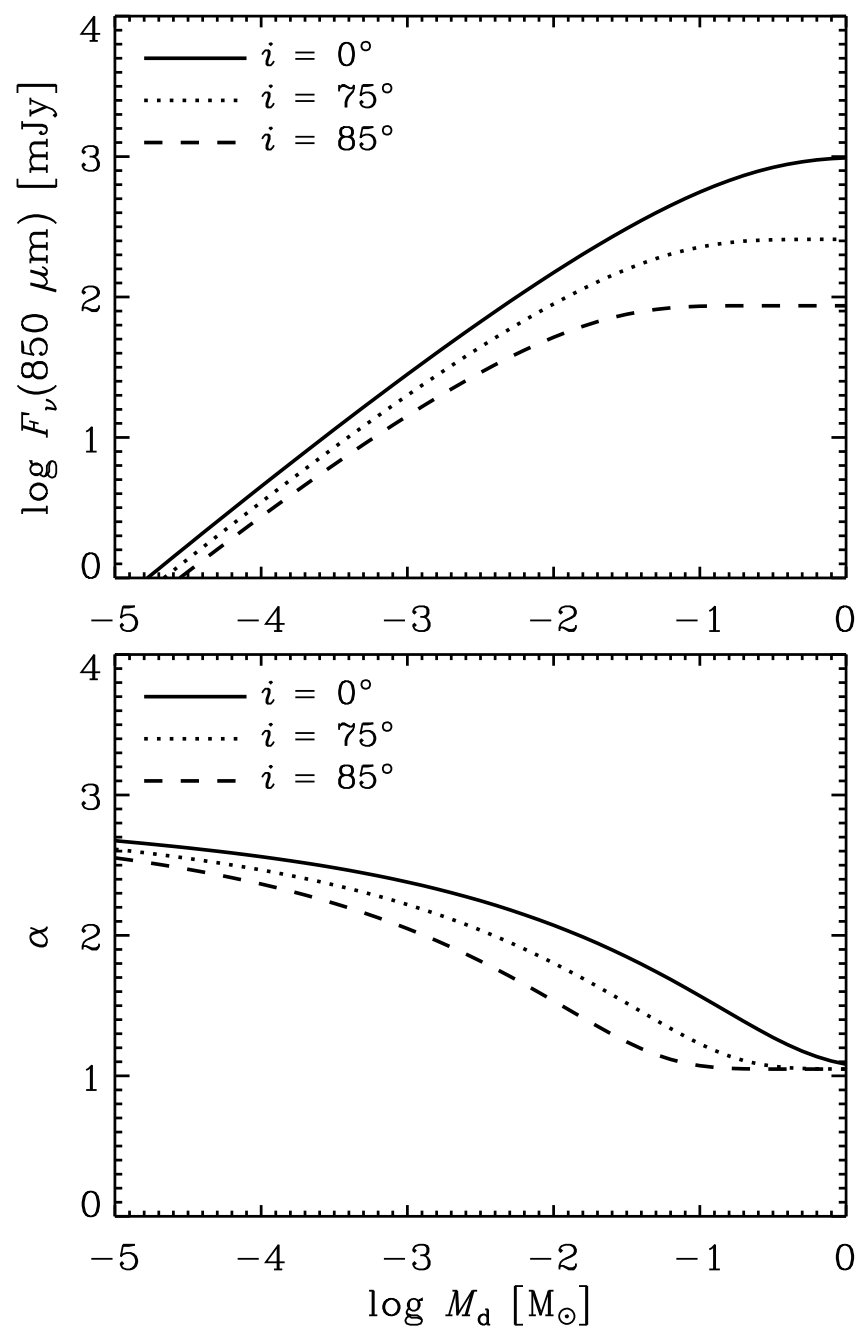

Fig. 14.- Illustration of the effect on the relationships between the disk mass and $850 \mu \mathrm{m}$ flux density or submillimeter continuum slope introduced by increasing the inclination angle of a fiducial circumstellar disk (the median disk model described in the text). Higher inclination angles (closer to edge-on disks) produce lower flux densities and continuum slopes for a given disk mass. 

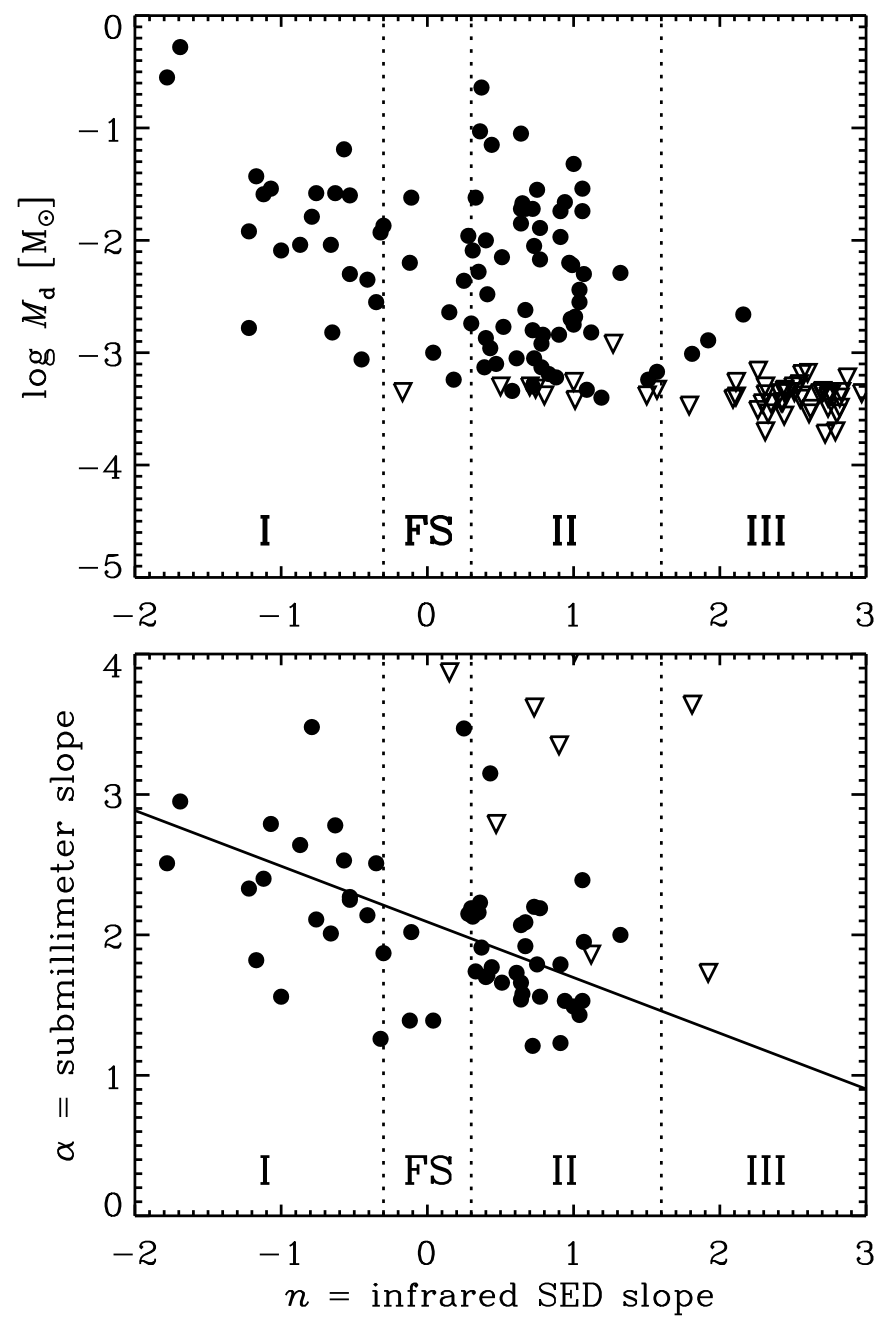

Fig. 15.- Relationship between the disk mass (top) or submillimeter continuum slope (bottom: $\alpha$ defined by $F_{\nu} \propto \nu^{\alpha}$ ) and the slope of the infrared SED from $\sim 2$ to $60 \mu \mathrm{m}$, defined as the index $n$ such that $\nu F_{\nu} \propto \nu^{n}$. The vertical dotted lines mark the borders between various SED classifications. Filled circles are detections and open triangles are 3- $\sigma$ upper limits. There is no direct correlation between $n$ and $\log M_{d}$. A correlation (3.7- $\sigma$ with Spearman rank coefficient of -0.50) is seen between $n$ and $\alpha$, where the best-fit linear relationship has been overlaid as a solid line: $\alpha=-0.40( \pm 0.04) n+2.09( \pm 0.03)$. The rms residual dispersion around the best-fit line is 0.24 , which is roughly the $1-\sigma$ error on $\alpha$ expected from absolute flux calibration uncertainties. 


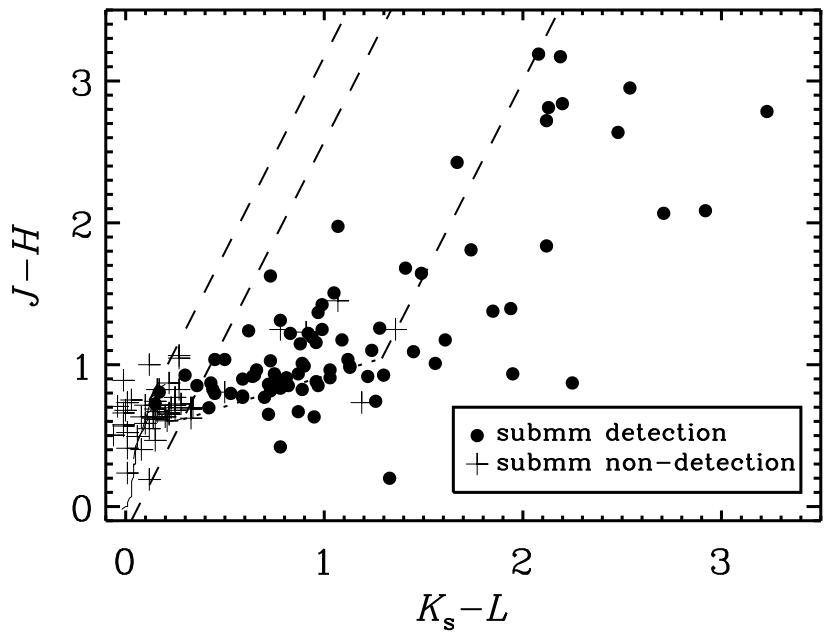

Fig. 16. - A near-infrared color-color diagram of the sample constructed from 2MASS data and $L$ band data from the literature. The solid curves mark the intrinsic colors of dwarf and giant stars. Dashed lines denote the reddening vectors based on the extinction law of Cohen et al. (1981), converted to the 2MASS photometric system. The dotted line corresponds to the classical T Tauri star locus derived by Meyer, Calvet, \& Hilldenbrand (1997). Filled circles are sources which are detected for at least 1 submillimeter wavelength (between $350 \mu \mathrm{m}$ and $1.3 \mathrm{~mm}$ ), and crosses are undetected. There are 6 objects with a near-infrared excess but no submillimeter detection which are addressed in the Appendix. The 4 objects with no infrared excess but a submillimeter detection imply that the timescales for dissipation of the inner and outer disk are similar. 

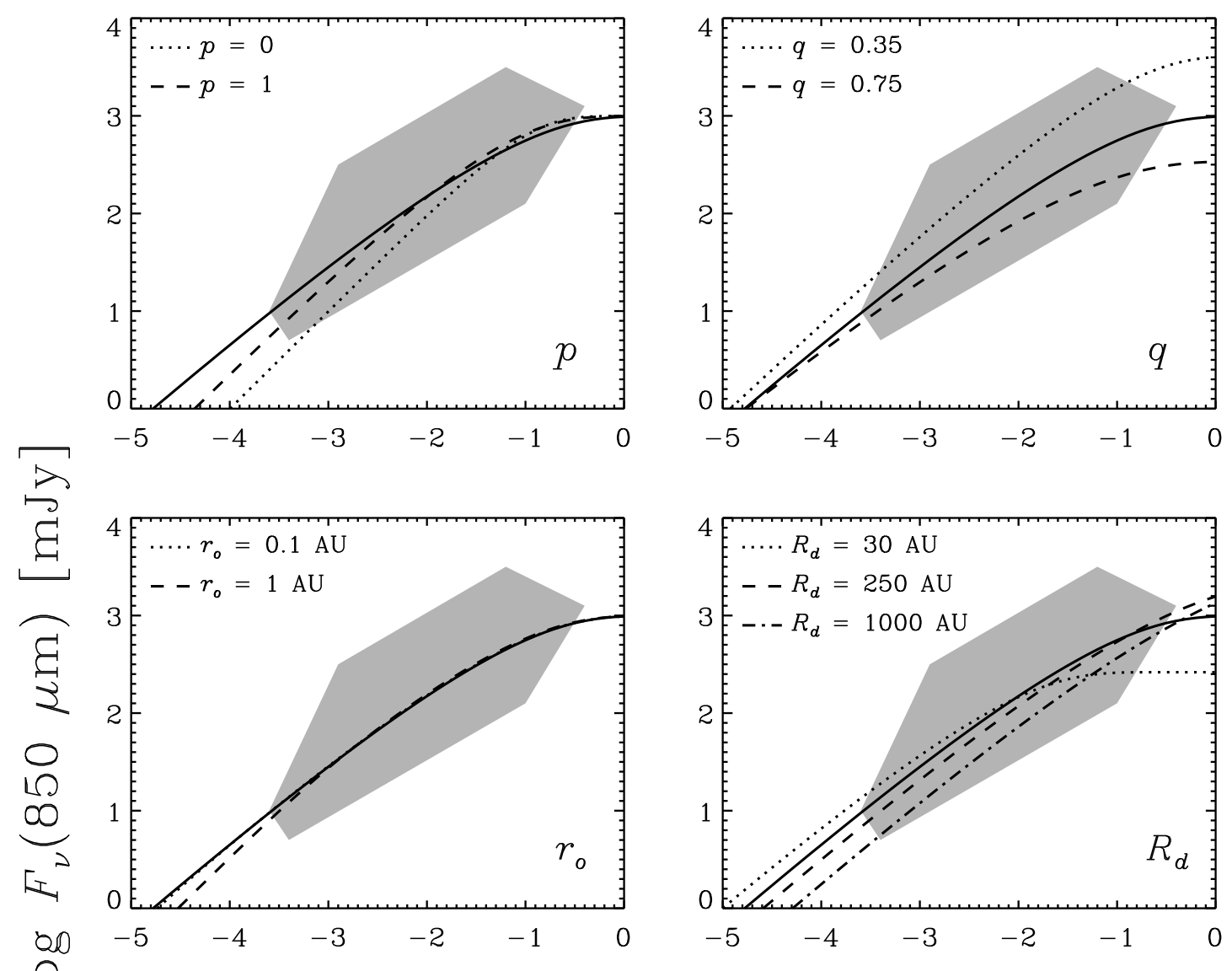

○
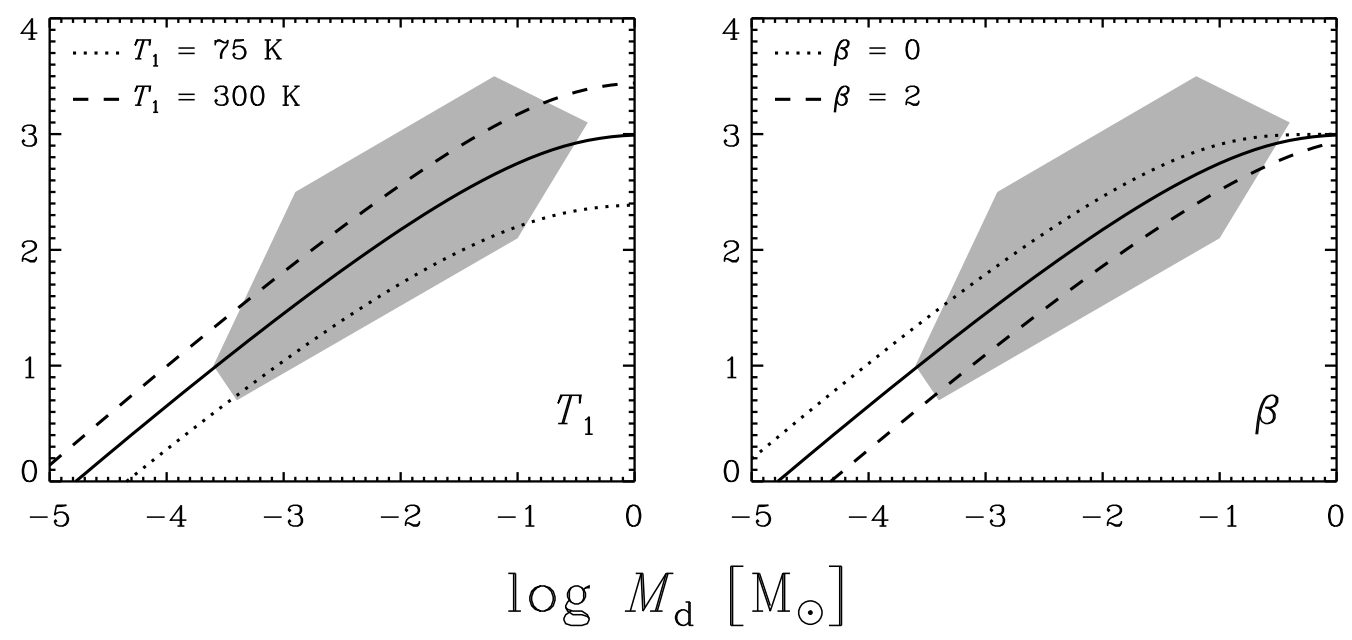

Fig. 17.- The effects of reasonable variations of the SED model parameters on the relationship between the $850 \mu \mathrm{m}$ flux density and the disk mass. The solid curve in each panel denotes the relationship for a fiducial parameter set: $i=0^{\circ}, p=1.5, q=0.6, r_{\circ}=0.01 \mathrm{AU}, R_{d}=100 \mathrm{AU}$, $T_{1}=150 \mathrm{~K}$, and $\beta=1$. The parameter being varied from this fiducial set is indicated in the lower right corner, and the different curves are marked in each panel. The shaded area is representative of the data values (see Figure 4). The temperature profile plays the dominant role in determining the relationship between $F_{\nu}$ and $M_{d}$. 

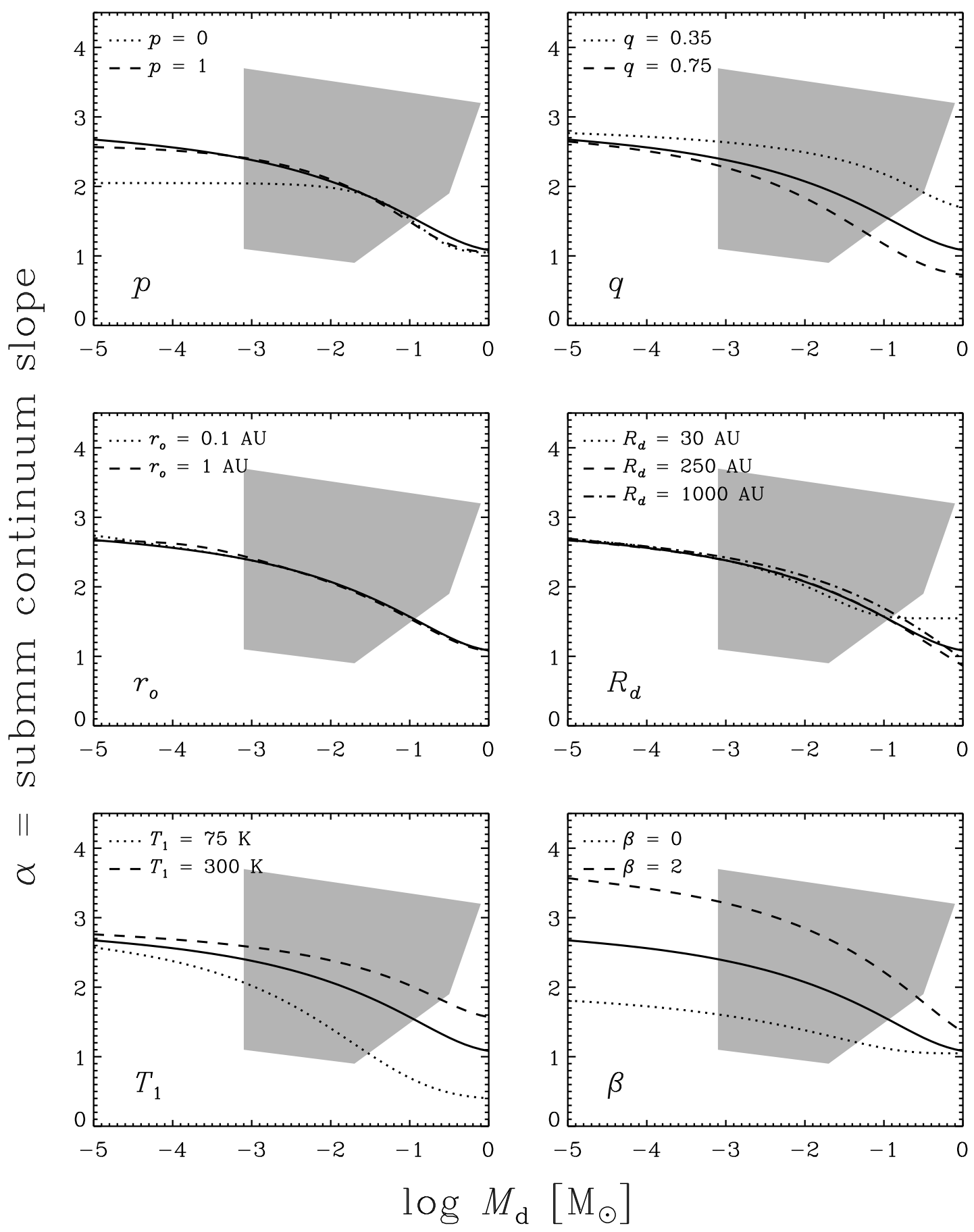

Fig. 18. - The effects of reasonable variations of the SED model parameters on the relationship between the submillimeter continuum slope and the disk mass for a fixed opacity index $\beta=1$. The solid curve in each panel denotes the relationship for a fiducial parameter set: $i=0^{\circ}, p=1.5$, $q=0.6, r_{\circ}=0.01 \mathrm{AU}, R_{d}=100 \mathrm{AU}, T_{1}=150 \mathrm{~K}$, and $\beta=1$. The parameter being varied from this fiducial set is indicated in the lower left corner, and the different curves are marked in each panel. The shaded area is representative of the data values. The lower right panel shows that $\beta$ is the dominant factor in setting the $\alpha-M_{d}$ relationship, but the temperature profile also has a significant effect. 


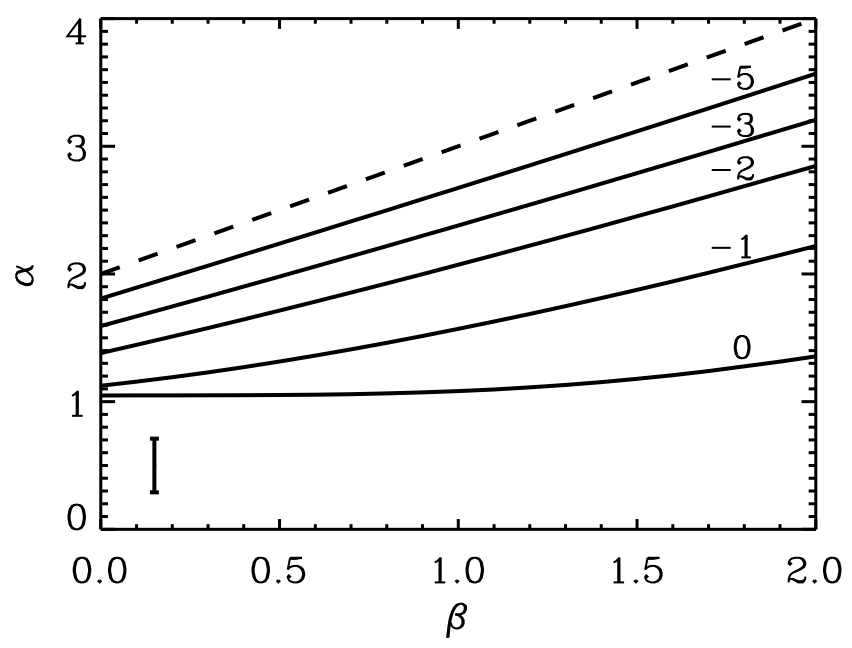

Fig. 19. - Submillimeter continuum slopes ( $\alpha$ : defined by $\left.F_{\nu} \propto \nu^{\alpha}\right)$ between $350 \mu \mathrm{m}$ and $1.3 \mathrm{~mm}$ from the fiducial disk model as a function of the power law index of the opacity $(\beta)$ for various input disk masses. Each curve is labeled with the logarithm of the disk mass. The dashed line marks the nominal relationship $\beta=\alpha-2$ for optically thin emission in the Rayleigh-Jeans limit. The relationship between $\beta$ and $\alpha$ is linear for disk masses less than $\sim 0.1 \mathrm{M}_{\odot}$, but decreased relative to the optically thin case due to the fraction of emission (particularly at the shortest wavelengths) which is optically thick. The error bar in the lower left corner shows the systematic uncertainty in $\alpha$ from absolute flux calibration errors. 

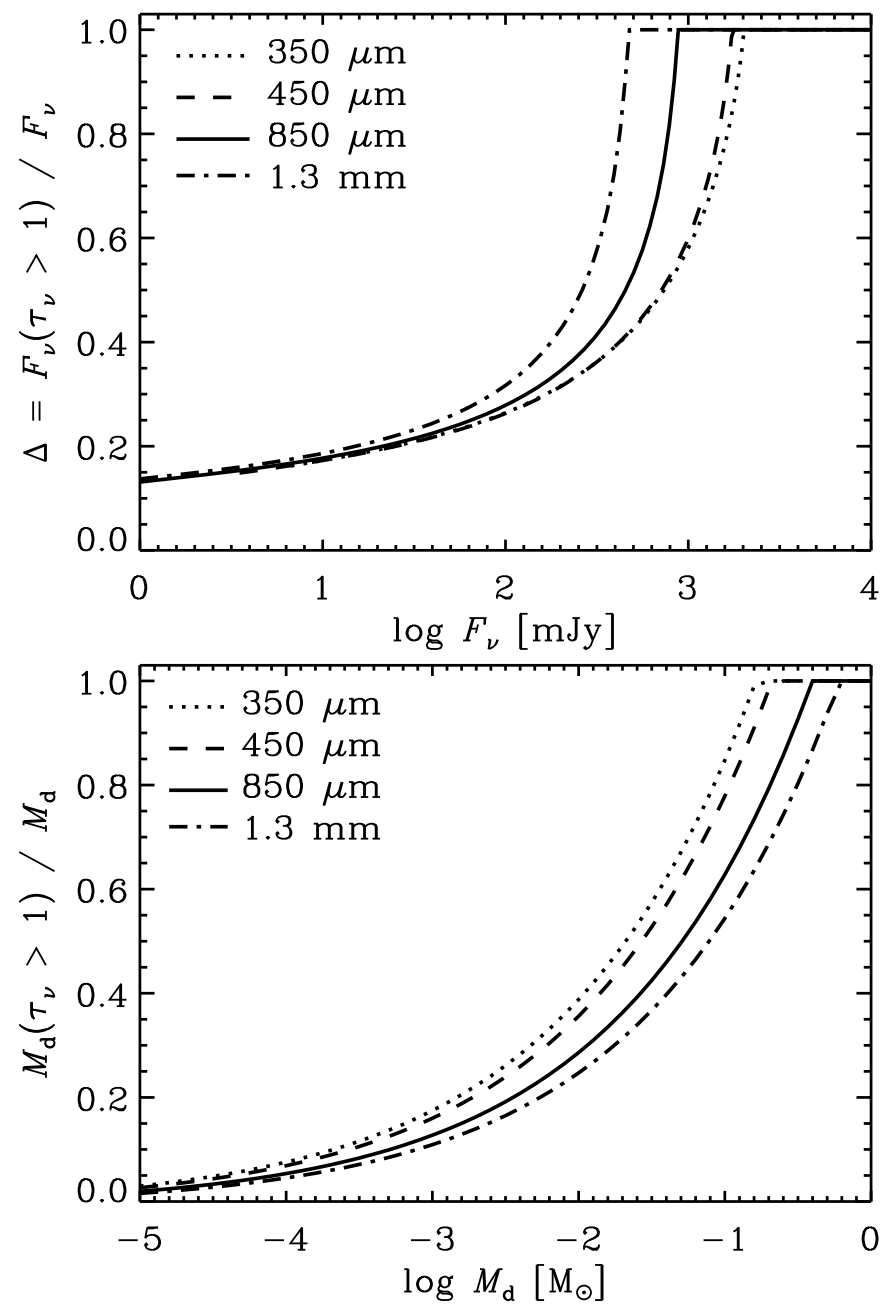

Fig. 20.- (top): The fraction of the submillimeter flux density which is from optically thick emission $(\Delta)$ as a function of the total flux density for the fiducial disk model. The various curves represent different wavelengths: $1.3 \mathrm{~mm}$ (dash-dotted), $850 \mu \mathrm{m}$ (solid), $450 \mu \mathrm{m}$ (dashed), and $350 \mu \mathrm{m}$ (dotted). (bottom): The fraction of the disk mass which gives rise to optically thick submillimeter continuum emission at various wavelengths as a function of the total disk mass. 
Table 1. Submillimeter Properties of Taurus-Auriga Disks ${ }^{\mathrm{a}}$

\begin{tabular}{|c|c|c|c|c|c|c|c|c|c|}
\hline \multirow[b]{2}{*}{$\begin{array}{l}\text { Object } \\
(1)\end{array}$} & \multirow[b]{2}{*}{$\begin{array}{l}\text { SED } \\
(2)\end{array}$} & \multirow[b]{2}{*}{$\begin{array}{l}\mathrm{SpT} \\
(3)\end{array}$} & \multicolumn{4}{|c|}{$F_{\nu}[\mathrm{mJy}]$} & \multirow[b]{2}{*}{$\begin{array}{c}M_{d}\left[\mathrm{M}_{\odot}\right] \\
\quad(8)\end{array}$} & \multirow[b]{2}{*}{$\begin{array}{c}\alpha \\
(9)\end{array}$} & \multirow[b]{2}{*}{$\begin{array}{c}\text { notes }^{b} \\
(10)\end{array}$} \\
\hline & & & $\begin{array}{c}\lambda=350 \mu \mathrm{m} \\
(4)\end{array}$ & $\begin{array}{c}\lambda=450 \mu \mathrm{m} \\
(5)\end{array}$ & $\begin{array}{c}\lambda=850 \mu \mathrm{m} \\
(6)\end{array}$ & $\begin{array}{c}\lambda=1.3 \mathrm{~mm} \\
(7)\end{array}$ & & & \\
\hline $04016+2610$ & I & $\cdots$ & $12477 \pm 193$ & $\cdots$ & $\cdots$ & $130 \pm 5$ & 0.02 & $3.48 \pm 0.24$ & 5,8 \\
\hline $04113+2758$ & II & M2 & $7621 \pm 219$ & $\ldots$ & $\ldots$ & $410 \pm 40$ & 0.09 & $2.23 \pm 0.24$ & 5 \\
\hline $04154+2823$ & FS & $\cdots$ & $440 \pm 83$ & $495 \pm 185$ & $140 \pm 6$ & $\ldots$ & 0.006 & $1.39 \pm 0.27$ & $\cdots$ \\
\hline $04166+2706$ & I & $\cdots$ & $6937 \pm 93$ & $\ldots$ & $\ldots$ & $180 \pm 8$ & 0.03 & $2.78 \pm 0.24$ & 5,8 \\
\hline $04169+2702$ & I & $\ldots$ & $7344 \pm 152$ & $\ldots$ & $\ldots$ & $190 \pm 9$ & 0.03 & $2.79 \pm 0.24$ & 5,8 \\
\hline $04239+2436$ & I & $\cdots$ & $1144 \pm 20$ & $<660$ & $207 \pm 9$ & $80 \pm 10$ & 0.009 & $2.01 \pm 0.24$ & 5,8 \\
\hline $04248+2612$ & I & M2 & $1178 \pm 30$ & $\ldots$ & $\ldots$ & $60 \pm 7$ & 0.005 & $2.27 \pm 0.21$ & 5,8 \\
\hline $04260+2642$ & I & K6 & $546 \pm 21$ & $\cdots$ & $\cdots$ & $105 \pm 10$ & 0.01 & $1.26 \pm 0.24$ & 5 \\
\hline $04278+2253$ & II & $\mathrm{F} 1$ & $\ldots$ & $<687$ & $36 \pm 7$ & $\ldots$ & 0.002 & $<4.64$ & $\cdots$ \\
\hline $04295+2251$ & FS & $\ldots$ & $1338 \pm 25$ & $\ldots$ & $\ldots$ & $115 \pm 10$ & 0.01 & $1.87 \pm 0.24$ & 5,8 \\
\hline $04301+2608$ & II & $\cdots$ & $\ldots$ & $<351$ & $18 \pm 6$ & $\ldots$ & 0.0009 & $<4.67$ & $\cdots$ \\
\hline $04302+2247$ & I & $\ldots$ & $2869 \pm 21$ & $\ldots$ & $\ldots$ & $180 \pm 10$ & 0.03 & $2.11 \pm 0.24$ & 5,8 \\
\hline $04325+2402$ & I & $\cdots$ & $\ldots$ & $606 \pm 185$ & $186 \pm 11$ & $110 \pm 7$ & 0.008 & $1.56 \pm 0.34$ & 5,8 \\
\hline $04361+2547$ & I & $\cdots$ & $\cdots$ & $1302 \pm 168$ & $275 \pm 8$ & $110 \pm 8$ & 0.01 & $2.33 \pm 0.30$ & 5,8 \\
\hline $04365+2535$ & I & $\cdots$ & $\cdots$ & $2928 \pm 230$ & $622 \pm 13$ & $230 \pm 10$ & 0.03 & $2.40 \pm 0.30$ & 5,8 \\
\hline $04368+2557$ & I & $\cdots$ & $\cdots$ & $2849 \pm 222$ & $895 \pm 11$ & $\ldots$ & 0.04 & $1.82 \pm 0.42$ & 8 \\
\hline $04381+2540$ & I & $\cdots$ & $\ldots$ & $1152 \pm 279$ & $208 \pm 11$ & $70 \pm 9$ & 0.009 & $2.64 \pm 0.30$ & 5,8 \\
\hline AA Tau & II & K7 & $825 \pm 50$ & $415 \pm 84$ & $144 \pm 5$ & $88 \pm 9$ & 0.01 & $1.56 \pm 0.20$ & 3,6 \\
\hline AB Aur & II & A0 & $8930 \pm 1410$ & $3820 \pm 570$ & $359 \pm 67$ & $103 \pm 18$ & 0.004 & $3.47 \pm 0.20$ & 2 \\
\hline Anon 1 & III & M0 & $\ldots$ & $<79$ & $<8$ & $<14$ & $<0.0004$ & $\ldots$ & 4 \\
\hline BP Tau & II & $\mathrm{K} 7$ & $\cdots$ & $<456$ & $130 \pm 7$ & $47 \pm 0.7$ & 0.02 & $2.39 \pm 0.53$ & 2 \\
\hline CIDA-2 & III & M5 & $\cdots$ & $<165$ & $<14$ & $\ldots$ & $<0.0007$ & $\ldots$ & $\cdots$ \\
\hline CIDA-3 & II & M2 & $\ldots$ & $<94$ & $<9$ & $\ldots$ & $<0.0004$ & $\ldots$ & $\ldots$ \\
\hline CIDA-7 & II & M3 & $\cdots$ & $990 \pm 330$ & $38 \pm 8$ & $\cdots$ & 0.002 & $5.13 \pm 0.62$ & 7 \\
\hline CIDA-8 & II & M4 & $\ldots$ & $<80$ & $27 \pm 3$ & $\cdots$ & 0.001 & $<1.73$ & $\ldots$ \\
\hline CIDA-9 & II & M0 & $\cdots$ & $843 \pm 259$ & $71 \pm 7$ & $\cdots$ & 0.003 & $3.89 \pm 0.51$ & $\cdots$ \\
\hline CIDA-10 & III & M4 & $\cdots$ & $<94$ & $<11$ & $\cdots$ & $<0.0005$ & $\ldots$ & $\cdots$ \\
\hline CIDA-11 & II & M3 & $\cdots$ & $<87$ & $<8$ & $\cdots$ & $<0.0004$ & $\cdots$ & $\cdots$ \\
\hline CIDA-12 & II & M4 & $\ldots$ & $<88$ & $<7$ & $\ldots$ & $<0.0004$ & $\ldots$ & $\cdots$ \\
\hline CI Tau & II & $\mathrm{K} 7$ & $1725 \pm 55$ & $846 \pm 89$ & $324 \pm 6$ & $190 \pm 17$ & 0.03 & $1.79 \pm 0.21$ & 3,6 \\
\hline $\mathrm{CoKu} \mathrm{Tau/1}$ & II & M0 & $\ldots$ & $<522$ & $35 \pm 7$ & $<12$ & 0.002 & $<4.25$ & 4 \\
\hline $\mathrm{CoKu} \mathrm{Tau/3}$ & II & M1 & $\ldots$ & $<104$ & $<8$ & $<16$ & $<0.0004$ & $\ldots$ & 4 \\
\hline $\mathrm{CoKu} \mathrm{Tau} / 4$ & II & M2 & $\ldots$ & $<131$ & $9.0 \pm 2.9$ & $<15$ & 0.0005 & $<4.21$ & 4 \\
\hline CW Tau & II & K2 & $1230 \pm 102$ & $<312$ & $66 \pm 6$ & $96 \pm 8$ & 0.002 & $2.09 \pm 0.24$ & 3,6 \\
\hline CX Tau & II & M3 & $\ldots$ & $<570$ & $25 \pm 6$ & $<40$ & 0.001 & $<4.92$ & 3 \\
\hline CY Tau & II & M1 & $<1839$ & $<210$ & $140 \pm 5$ & $133 \pm 11$ & 0.006 & $0.75 \pm 0.56$ & $3,6,7$ \\
\hline CZ Tau & II & M2 & $\cdots$ & $<262$ & $<9$ & $<30$ & $<0.0004$ & $\ldots$ & 3 \\
\hline DD Tau & II & M1 & $\cdots$ & $\ldots$ & $<42$ & $17 \pm 4$ & 0.0007 & $\ldots$ & 4 \\
\hline DE Tau & II & M2 & $\ldots$ & $<291$ & $90 \pm 7$ & $36 \pm 5$ & 0.005 & $2.16 \pm 0.40$ & 4 \\
\hline DF Tau & II & M1 & $\ldots$ & $<304$ & $8.8 \pm 1.9$ & $<25$ & 0.0004 & $<5.57$ & 1,3 \\
\hline DG Tau & FS & K7 & $5173 \pm 94$ & $3950 \pm 350$ & $1100 \pm 100$ & $700 \pm 130$ & 0.02 & $2.02 \pm 0.12$ & 2,6 \\
\hline DH Tau & II & M1 & $261 \pm 9$ & $\ldots$ & $57 \pm 9$ & $<57$ & 0.003 & $1.71 \pm 0.33$ & 3 \\
\hline DK Tau & II & K7 & $\ldots$ & $<419$ & $80 \pm 10$ & $35 \pm 7$ & 0.005 & $1.95 \pm 0.55$ & 3 \\
\hline DL Tau & II & K7 & $1390 \pm 180$ & $1280 \pm 170$ & $440 \pm 40$ & $230 \pm 14$ & 0.09 & $1.54 \pm 0.15$ & $2,3,6$ \\
\hline
\end{tabular}


Table 1-Continued

\begin{tabular}{|c|c|c|c|c|c|c|c|c|c|}
\hline \multirow[b]{2}{*}{$\begin{array}{l}\text { Object } \\
(1)\end{array}$} & \multirow[b]{2}{*}{$\begin{array}{l}\text { SED } \\
(2)\end{array}$} & \multirow[b]{2}{*}{$\begin{array}{c}\text { SpT } \\
(3)\end{array}$} & \multicolumn{4}{|c|}{$F_{\nu}[\mathrm{mJy}]$} & \multirow[b]{2}{*}{$\begin{array}{c}M_{d}\left[\mathrm{M}_{\odot}\right] \\
(8)\end{array}$} & \multirow[b]{2}{*}{$\begin{array}{c}\alpha \\
(9)\end{array}$} & \multirow[b]{2}{*}{$\begin{array}{c}\text { notes }^{b} \\
(10)\end{array}$} \\
\hline & & & $\begin{array}{c}\lambda=350 \mu \mathrm{m} \\
(4)\end{array}$ & $\begin{array}{c}\lambda=450 \mu \mathrm{m} \\
(5)\end{array}$ & $\begin{array}{c}\lambda=850 \mu \mathrm{m} \\
(6)\end{array}$ & $\begin{array}{c}\lambda=1.3 \mathrm{~mm} \\
(7)\end{array}$ & & & \\
\hline DM Tau & II & M1 & $1077 \pm 49$ & $\ldots$ & $237 \pm 12$ & $109 \pm 13$ & 0.02 & $1.74 \pm 0.24$ & 3 \\
\hline DN Tau & II & M0 & $615 \pm 64$ & $<703$ & $201 \pm 7$ & $84 \pm 13$ & 0.03 & $1.53 \pm 0.23$ & 3,6 \\
\hline DO Tau & II & M0 & $\ldots$ & $734 \pm 50$ & $258 \pm 42$ & $136 \pm 11$ & 0.007 & $1.66 \pm 0.19$ & $1,3,6$ \\
\hline DP Tau & II & M1 & $\ldots$ & $<279$ & $<10$ & $<27$ & $<0.0005$ & $\ldots$ & 3 \\
\hline DQ Tau & II & M0 & $244 \pm 76$ & $<861$ & $208 \pm 8$ & $91 \pm 9$ & 0.02 & $1.21 \pm 0.27$ & 3,6 \\
\hline DR Tau & II & $\ldots$ & $\ldots$ & $2380 \pm 172$ & $533 \pm 7$ & $159 \pm 11$ & 0.02 & $2.07 \pm 0.19$ & $1,3,6$ \\
\hline DS Tau & II & K5 & $\ldots$ & $<342$ & $39 \pm 4$ & $25 \pm 6$ & 0.006 & $1.05 \pm 0.61$ & $1,4,7$ \\
\hline FF Tau & III & $\mathrm{K} 7$ & $\ldots$ & $<43$ & $<4$ & $<27$ & $<0.0002$ & $\ldots$ & 1,3 \\
\hline FM Tau & II & M0 & $<349$ & $<442$ & $32 \pm 8$ & $<36$ & 0.002 & $<4.13$ & 3 \\
\hline FO Tau & II & M2 & $\ldots$ & $<199$ & $13 \pm 3$ & $<14$ & 0.0006 & $<4.29$ & 1,4 \\
\hline FQ Tau & II & M2 & $\cdots$ & $574 \pm 170$ & $28 \pm 7$ & $<40$ & 0.001 & $4.75 \pm 0.61$ & 3,7 \\
\hline FS Tau & FS & M1 & $\ldots$ & $<576$ & $49 \pm 6$ & $<35$ & 0.002 & $<3.87$ & 3 \\
\hline FT Tau & II & $\ldots$ & $1106 \pm 82$ & $437 \pm 56$ & $121 \pm 5$ & $130 \pm 14$ & 0.01 & $1.66 \pm 0.20$ & 3,6 \\
\hline FV Tau & II & K5 & $\ldots$ & $402 \pm 90$ & $48 \pm 5$ & $15 \pm 4$ & 0.001 & $3.15 \pm 0.34$ & 1,4 \\
\hline FV Tau/c & II & M4 & $\ldots$ & $<355$ & $<25$ & $<16$ & $<0.001$ & $\ldots$ & 4 \\
\hline FW Tau & III & M4 & $\ldots$ & $<35$ & $4.5 \pm 1.1$ & $<15$ & 0.0002 & $<3.23$ & 1,3 \\
\hline FX Tau & II & M1 & $\ldots$ & $<169$ & $17 \pm 3$ & $<30$ & 0.0009 & $<3.62$ & 3 \\
\hline FY Tau & II & $\mathrm{K} 7$ & $\ldots$ & $<297$ & $<27$ & $16 \pm 5$ & 0.0007 & $\ldots$ & 3 \\
\hline FZ Tau & II & M0 & $\ldots$ & $<273$ & $29 \pm 7$ & $23 \pm 7$ & 0.002 & $0.55 \pm 0.89$ & 3,7 \\
\hline GG Tau & II & $\mathrm{K} 7$ & $6528 \pm 153$ & $2726 \pm 250$ & $1255 \pm 57$ & $593 \pm 53$ & 0.2 & $1.91 \pm 0.12$ & $1,3,6$ \\
\hline GH Tau & II & M2 & $\ldots$ & $<309$ & $15 \pm 3$ & $<30$ & 0.0007 & $<4.76$ & 1,3 \\
\hline GK Tau & II & K7 & $\ldots$ & $\ldots$ & $33 \pm 7$ & $<21$ & 0.002 & $\ldots$ & 3 \\
\hline GM Aur & II & K3 & $3419 \pm 133$ & $\ldots$ & $\ldots$ & $253 \pm 12$ & 0.03 & $2.25 \pm 0.23$ & 3,6 \\
\hline GN Tau & II & $\cdots$ & $\ldots$ & $<187$ & $12 \pm 3$ & $<50$ & 0.0006 & $<4.32$ & 1,3 \\
\hline GO Tau & II & M0 & $274 \pm 26$ & $594 \pm 185$ & $173 \pm 7$ & $83 \pm 12$ & 0.07 & $1.77 \pm 0.33$ & 3,6 \\
\hline GV Tau & I & K3 & $1676 \pm 137$ & $1808 \pm 121$ & $282 \pm 5$ & $87 \pm 4$ & 0.003 & $2.51 \pm 0.20$ & 4,6 \\
\hline Haro 6-13 & FS & M0 & $2729 \pm 171$ & $1400 \pm 180$ & $395 \pm 56$ & $124 \pm 13$ & 0.01 & $2.15 \pm 0.18$ & $1,3,6$ \\
\hline Haro 6-28 & FS & M5 & $\ldots$ & $<2636$ & $11 \pm 3$ & $<14$ & 0.0006 & $<8.55$ & 1,4 \\
\hline Haro 6-37 & II & K6 & $\cdots$ & $536 \pm 204$ & $245 \pm 7$ & $<88$ & 0.01 & $1.23 \pm 0.62$ & 4 \\
\hline Haro 6-39 & II & $\ldots$ & $\ldots$ & $<903$ & $36 \pm 6$ & $24 \pm 6$ & 0.002 & $0.95 \pm 0.72$ & 3,7 \\
\hline HBC 347 & III & $\mathrm{K} 1$ & $\ldots$ & $<138$ & $<9$ & $\ldots$ & $<0.0004$ & $\ldots$ & 4 \\
\hline HBC 351 & III & K5 & $\ldots$ & $<166$ & $<11$ & $<14$ & $<0.0005$ & $\ldots$ & 4 \\
\hline HBC $352 / 353$ & III & G0 & $\cdots$ & $<81$ & $<9$ & $<12$ & $<0.0005$ & $\cdots$ & 4 \\
\hline HBC $354 / 355$ & III & K3 & $\ldots$ & $<71$ & $<7$ & $<16$ & $<0.0004$ & $\ldots$ & 4 \\
\hline HBC $356 / 357$ & III & $\mathrm{K} 2$ & $\ldots$ & $<69$ & $<9$ & $<16$ & $<0.0004$ & $\ldots$ & 4 \\
\hline HBC $358 / 359$ & III & M2 & $\cdots$ & $<72$ & $<9$ & $<13$ & $<0.0005$ & $\ldots$ & 4 \\
\hline HBC $360 / 361$ & III & M3 & $\cdots$ & $<243$ & $<14$ & $<26$ & $<0.0007$ & $\cdots$ & 1,4 \\
\hline HBC 362 & III & M2 & $\cdots$ & $<108$ & $<8$ & $<14$ & $<0.0004$ & $\cdots$ & 4 \\
\hline HBC 372 & III & K5 & $\ldots$ & $<173$ & $<8$ & $<14$ & $<0.0004$ & $\ldots$ & 4 \\
\hline HBC 376 & III & K7 & $\ldots$ & $<49$ & $<6$ & $<14$ & $<0.0003$ & $\ldots$ & 1,4 \\
\hline HBC 388 & III & $\mathrm{K} 1$ & $\ldots$ & $<54$ & $<6$ & $<16$ & $<0.0003$ & $\ldots$ & 1,4 \\
\hline HBC 392 & III & K5 & $\cdots$ & $<141$ & $<6$ & $<18$ & $<0.0003$ & $\cdots$ & 1,4 \\
\hline HBC 407 & III & G8 & $\ldots$ & $<146$ & $<9$ & $<64$ & $<0.0004$ & $\ldots$ & 4 \\
\hline HBC 412 & III & M2 & $\ldots$ & $<200$ & $<9$ & $<16$ & $<0.0004$ & $\ldots$ & 4 \\
\hline
\end{tabular}


Table 1-Continued

\begin{tabular}{|c|c|c|c|c|c|c|c|c|c|}
\hline \multirow[b]{2}{*}{$\begin{array}{l}\text { Object } \\
(1)\end{array}$} & \multirow[b]{2}{*}{$\begin{array}{l}\text { SED } \\
(2)\end{array}$} & \multirow[b]{2}{*}{$\begin{array}{c}\text { SpT } \\
(3)\end{array}$} & \multicolumn{4}{|c|}{$F_{\nu}[\mathrm{mJy}]$} & \multirow[b]{2}{*}{$\begin{array}{c}M_{d}\left[\mathrm{M}_{\odot}\right] \\
\quad(8)\end{array}$} & \multirow[b]{2}{*}{$\begin{array}{c}\alpha \\
(9)\end{array}$} & \multirow[b]{2}{*}{$\begin{array}{c}\text { notes }^{b} \\
(10)\end{array}$} \\
\hline & & & $\begin{array}{c}\lambda=350 \mu \mathrm{m} \\
(4)\end{array}$ & $\begin{array}{c}\lambda=450 \mu \mathrm{m} \\
(5)\end{array}$ & $\begin{array}{c}\lambda=850 \mu \mathrm{m} \\
(6)\end{array}$ & $\begin{array}{c}\lambda=1.3 \mathrm{~mm} \\
(7)\end{array}$ & & & \\
\hline HBC 427 & III & $\mathrm{K} 7$ & $\cdots$ & $<1516$ & $<14$ & $\ldots$ & $<0.0007$ & $\cdots$ & 1 \\
\hline HD 283572 & III & G5 & $\cdots$ & $<216$ & $<9$ & $<15$ & $<0.0004$ & $\cdots$ & 4 \\
\hline HD 283759 & III & F3 & $\ldots$ & $<149$ & $<10$ & $<35$ & $<0.0005$ & $\ldots$ & 3 \\
\hline HK Tau & FS & M1 & $680 \pm 114$ & $\cdots$ & $\ldots$ & $41 \pm 5$ & 0.004 & $2.14 \pm 0.21$ & 3,6 \\
\hline HL Tau & $\mathrm{I}$ & $\mathrm{K} 7$ & $23888 \pm 149$ & $10400 \pm 1400$ & $2360 \pm 90$ & $880 \pm 19$ & 0.06 & $2.53 \pm 0.13$ & $2,3,6,8$ \\
\hline HN Tau & II & K5 & $\ldots$ & $<171$ & $29 \pm 3$ & $<15$ & 0.0008 & $<2.79$ & 1,4 \\
\hline HO Tau & II & M1 & $\ldots$ & $<567$ & $44 \pm 6$ & $<30$ & 0.002 & $<4.02$ & 3 \\
\hline HP Tau & FS & K3 & $386 \pm 63$ & $\ldots$ & $\ldots$ & $62 \pm 6$ & 0.001 & $1.39 \pm 0.24$ & 3 \\
\hline HQ Tau & III & $\cdots$ & $\ldots$ & $<221$ & $11 \pm 3$ & $<45$ & 0.0005 & $<4.72$ & 1,3 \\
\hline Hubble 4 & III & K7 & $\cdots$ & $<89$ & $<9$ & $<25$ & $<0.0004$ & $\ldots$ & 3 \\
\hline HV Tau & II & M1 & $\cdots$ & $<519$ & $47 \pm 4$ & $40 \pm 6$ & 0.002 & $0.38 \pm 0.42$ & $1,4,7$ \\
\hline IC $2087 / \mathrm{IR}$ & II & $\ldots$ & $\ldots$ & $1365 \pm 130$ & $501 \pm 7$ & $\ldots$ & 0.02 & $1.58 \pm 0.42$ & $\ldots$ \\
\hline IP Tau & II & M0 & $\ldots$ & $<516$ & $34 \pm 5$ & $16 \pm 5$ & 0.003 & $1.80 \pm 0.81$ & 4,7 \\
\hline IQ Tau & II & M1 & $\cdots$ & $425 \pm 26$ & $178 \pm 3$ & $87 \pm 11$ & 0.02 & $1.53 \pm 0.30$ & 3,6 \\
\hline IS Tau & II & K7 & $\ldots$ & $<252$ & $30 \pm 3$ & $<20$ & 0.001 & $<3.35$ & 1,3 \\
\hline IT Tau & II & K2 & $\ldots$ & $<73$ & $22 \pm 3$ & $<33$ & 0.002 & $<1.86$ & 3 \\
\hline IW Tau & III & K7 & $\ldots$ & $<253$ & $<9$ & $<19$ & $<0.0004$ & $\ldots$ & 1,4 \\
\hline J1-4423 & III & M5 & $\cdots$ & $<52$ & $<8$ & $<11$ & $<0.0004$ & $\cdots$ & 4 \\
\hline J1-4872 & III & $\mathrm{K} 7$ & $\ldots$ & $<63$ & $<8$ & $<14$ & $<0.0004$ & $\ldots$ & 4 \\
\hline J1-507 & III & M4 & $\cdots$ & $<52$ & $<6$ & $<14$ & $<0.0003$ & $\ldots$ & 4 \\
\hline JH 56 & III & M1 & $\ldots$ & $<74$ & $<8$ & $<19$ & $<0.0004$ & $\cdots$ & 4 \\
\hline JH 108 & III & M1 & $\ldots$ & $<66$ & $<7$ & $<18$ & $<0.0004$ & $\ldots$ & 4 \\
\hline JH 112 & II & K6 & $\ldots$ & $\ldots$ & $30 \pm 10$ & $<18$ & 0.001 & $<5.51$ & 4 \\
\hline JH 223 & II & M2 & $\ldots$ & $<62$ & $<7$ & $<19$ & $<0.0003$ & $\ldots$ & 4 \\
\hline L1551-51 & III & $\mathrm{K} 7$ & $\cdots$ & $<201$ & $<13$ & $\ldots$ & $<0.0006$ & $\cdots$ & $\cdots$ \\
\hline L1551-55 & III & K7 & $\ldots$ & $<60$ & $<5$ & $<23$ & $<0.0003$ & $\ldots$ & 1,4 \\
\hline L1551 IRS5 & I & $\cdots$ & $100423 \pm 812$ & $\cdots$ & $\cdots$ & $1276 \pm 5$ & 0.5 & $2.95 \pm 0.17$ & 4,8 \\
\hline L1551 NE & I & $\ldots$ & $22826 \pm 715$ & $\ldots$ & $\ldots$ & $850 \pm 10$ & 0.3 & $2.51 \pm 0.24$ & 5 \\
\hline $\mathrm{LkCa} 1$ & III & M4 & $\cdots$ & $<89$ & $<8$ & $<14$ & $<0.0004$ & $\ldots$ & 4 \\
\hline $\mathrm{LkCa} 3$ & III & M1 & $\ldots$ & $<471$ & $<9$ & $<14$ & $<0.0004$ & $\ldots$ & 1,4 \\
\hline $\mathrm{LkCa} 4$ & III & $\mathrm{K} 7$ & $\cdots$ & $<37$ & $<4$ & $<14$ & $<0.0002$ & $\cdots$ & 1,4 \\
\hline $\mathrm{LkCa} 5$ & III & M2 & $\ldots$ & $<28$ & $<4$ & $<14$ & $<0.0002$ & $\ldots$ & 1,4 \\
\hline LkCa 7 & III & $\mathrm{K} 7$ & $\cdots$ & $<107$ & $<9$ & $\cdots$ & $<0.0004$ & $\cdots$ & $\cdots$ \\
\hline LkCa 14 & III & M0 & $\ldots$ & $<103$ & $<9$ & $<19$ & $<0.0004$ & $\ldots$ & 4 \\
\hline $\mathrm{LkCa} 15$ & II & K5 & $1235 \pm 80$ & $\ldots$ & $428 \pm 11$ & $167 \pm 6$ & 0.05 & $1.49 \pm 0.24$ & 4 \\
\hline LkCa 19 & III & K0 & $\ldots$ & $<90$ & $<10$ & $\ldots$ & 0.0005 & $\ldots$ & $\cdots$ \\
\hline $\mathrm{LkCa} 21$ & III & M3 & $\ldots$ & $<145$ & $<10$ & $<12$ & $<0.0005$ & $\ldots$ & 4 \\
\hline $\mathrm{LkH} \alpha 332 / \mathrm{G} 1$ & III & M1 & $\cdots$ & $<663$ & $12 \pm 3$ & $<14$ & 0.0006 & $<6.38$ & 1,4 \\
\hline $\mathrm{LkH} \alpha 332 / \mathrm{G} 2$ & III & $\mathrm{K} 7$ & $\ldots$ & $<1083$ & $<9$ & $<15$ & $<0.0005$ & $\ldots$ & 1,4 \\
\hline RW Aur & II & K3 & $305 \pm 32$ & $167 \pm 34$ & $79 \pm 4$ & $42 \pm 5$ & 0.004 & $1.43 \pm 0.21$ & 4 \\
\hline RY Tau & II & K1 & $2439 \pm 330$ & $1920 \pm 160$ & $560 \pm 30$ & $229 \pm 17$ & 0.02 & $1.79 \pm 0.17$ & $2,3,6$ \\
\hline SAO 76411 & III & G1 & $\cdots$ & $<102$ & $<9$ & $<14$ & $<0.0005$ & $\cdots$ & 4 \\
\hline SAO 76428 & III & F8 & $\cdots$ & $<123$ & $<12$ & $<14$ & $<0.0006$ & $\cdots$ & 4 \\
\hline St 34 & II & M3 & $\ldots$ & $<243$ & $<11$ & $<15$ & $<0.0005$ & $\ldots$ & 4 \\
\hline
\end{tabular}


Table 1-Continued

\begin{tabular}{|c|c|c|c|c|c|c|c|c|c|}
\hline \multirow[b]{2}{*}{$\begin{array}{l}\text { Object } \\
(1)\end{array}$} & \multirow[b]{2}{*}{$\begin{array}{c}\text { SED } \\
(2)\end{array}$} & \multirow[b]{2}{*}{$\begin{array}{c}\text { SpT } \\
(3)\end{array}$} & \multicolumn{4}{|c|}{$F_{\nu}[\mathrm{mJy}]$} & \multirow[b]{2}{*}{$\begin{array}{c}M_{d}\left[\mathrm{M}_{\odot}\right] \\
(8)\end{array}$} & \multirow[b]{2}{*}{$\begin{array}{c}\alpha \\
(9)\end{array}$} & \multirow[b]{2}{*}{$\begin{array}{c}\text { notes }^{b} \\
(10)\end{array}$} \\
\hline & & & $\begin{array}{c}\lambda=350 \mu \mathrm{m} \\
(4)\end{array}$ & $\lambda=450 \mu \mathrm{m}$ & $\lambda=850 \mu \mathrm{m}$ & $\begin{array}{c}\lambda=1.3 \mathrm{~mm} \\
(7)\end{array}$ & & & \\
\hline SU Aur & II & G2 & $\ldots$ & $251 \pm 40$ & $74 \pm 3$ & $<30$ & 0.0009 & $1.73 \pm 0.21$ & 3 \\
\hline $\mathrm{T}$ Tau & II & K0 & $8149 \pm 253$ & $1655 \pm 218$ & $628 \pm 17$ & $280 \pm 9$ & 0.008 & $2.13 \pm 0.30$ & $1,3,6,8$ \\
\hline UX Tau & II & K2 & $\ldots$ & $523 \pm 37$ & $173 \pm 3$ & $63 \pm 10$ & 0.005 & $2.00 \pm 0.30$ & 4 \\
\hline UY Aur & II & K7 & $542 \pm 77$ & $<523$ & $102 \pm 6$ & $29 \pm 6$ & 0.002 & $2.19 \pm 0.24$ & 4 \\
\hline UZ Tau & II & M1 & $1823 \pm 142$ & $1811 \pm 129$ & $560 \pm 7$ & $172 \pm 15$ & 0.02 & $1.92 \pm 0.20$ & $1,3,6$ \\
\hline V410 Tau & III & K3 & $\ldots$ & $<206$ & $7.2 \pm 1.8$ & $<30$ & $<0.0004$ & $<5.27$ & $1,3,7$ \\
\hline V710 Tau & II & M1 & $\ldots$ & $<495$ & $152 \pm 6$ & $60 \pm 7$ & 0.007 & $2.19 \pm 0.36$ & 4,6 \\
\hline V773 Tau & II & K3 & $\ldots$ & $<386$ & $9.2 \pm 2.9$ & $24 \pm 4$ & 0.0005 & $<5.88$ & $1,4,7$ \\
\hline V807 Tau & III & K7 & $\ldots$ & $<202$ & $20 \pm 3$ & $<18$ & 0.001 & $<3.64$ & 1,4 \\
\hline V819 Tau & III & $\mathrm{K} 7$ & $\ldots$ & $<317$ & $<9$ & $<9$ & $<0.0004$ & $\ldots$ & 1,4 \\
\hline V826 Tau & III & M0 & $\ldots$ & $<234$ & $<7$ & $<15$ & $<0.0004$ & $\ldots$ & 1,4 \\
\hline V827 Tau & III & $\mathrm{K} 7$ & $\ldots$ & $<147$ & $<6$ & $<19$ & $<0.0003$ & $\cdots$ & 1,4 \\
\hline V830 Tau & III & M0 & $\ldots$ & $<57$ & $<6$ & $<9$ & $<0.0003$ & $\ldots$ & 1,4 \\
\hline V836 Tau & II & $\mathrm{K} 7$ & $344 \pm 30$ & $231 \pm 43$ & $74 \pm 3$ & $37 \pm 6$ & 0.01 & $1.70 \pm 0.24$ & 4 \\
\hline V892 Tau & II & A0 & $4100 \pm 560$ & $2570 \pm 350$ & $638 \pm 54$ & $234 \pm 19$ & 0.009 & $2.20 \pm 0.19$ & 2,6 \\
\hline V927 Tau & III & M5 & $\ldots$ & $<1030$ & $<10$ & $<20$ & $<0.0005$ & $\ldots$ & 1,3 \\
\hline V928 Tau & III & M1 & $\ldots$ & $<258$ & $<8$ & $<11$ & $<0.0004$ & $\ldots$ & 1,4 \\
\hline V955 Tau & II & M0 & $\ldots$ & $<390$ & $14 \pm 2$ & $<19$ & 0.0005 & $<5.27$ & 1,4 \\
\hline VY Tau & II & M0 & $\ldots$ & $<225$ & $<10$ & $<17$ & $<0.0005$ & $\ldots$ & 1,4 \\
\hline Wa Tau/1 & III & Ko & $\ldots$ & $<55$ & $<6$ & $<19$ & $<0.0003$ & $\ldots$ & 1,4 \\
\hline ZZ Tau & III & M3 & $\ldots$ & $<251$ & $<8$ & $<15$ & $<0.0004$ & $\cdots$ & 1,3 \\
\hline
\end{tabular}

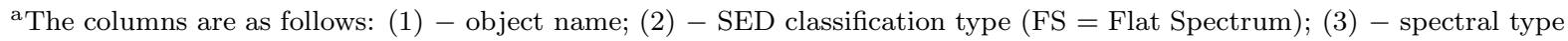
from the literature (see $\S 3.4$ ); (4) - $350 \mu \mathrm{m}$ flux density; (5) - $450 \mu \mathrm{m}$ flux density; (6) - $850 \mu \mathrm{m}$ flux density; (7) $-1.3 \mathrm{~mm}$ flux density values taken from the literature; (8) - logarithm of the disk mass (see §3.2); (9) - submillimeter continuum slope (see §3.3); (10) - notes on individual sources. All flux densities are measured in units of mJy. Upper limits are taken at the 3- $\sigma$ confidence level. Quoted errors are the 1- $\sigma$ rms noise levels and do not include systematic errors in the absolute flux calibration ( $\sim 25 \%$ at 350 and $450 \mu \mathrm{m}, \sim 10 \%$ at $850 \mu \mathrm{m}$, and $\sim 20 \%$ at $1.3 \mathrm{~mm}$ ).

${ }^{\mathrm{b}}$ The numbers in the notes column (10) refer to the following information: $1-450$ and $850 \mu \mathrm{m}$ data are from the JCMT SCUBA archive. The original data were taken at various times between 1997 and 2002 and were reduced in the same way described in $\S 2$ with slight modifications for the different filter set before 1999 November. 2 - Flux densities for V892 Tau (except 1.3 mm) and AB Aur are from Mannings (1994). Flux densities for HL Tau are taken from the long-term, repeated measurements at SCUBA (450 and $850 \mu \mathrm{m}$ : see http://www.jach.hawaii.edu/JCMT/continuum/calibration/sens/secondary_2004.html and Jenness et al. 2002). Flux densities for RY Tau (except $1.3 \mathrm{~mm}$ ), DG Tau (except $350 \mu \mathrm{m}$ ), and DL Tau (except $1.3 \mathrm{~mm}$ ) are taken from Mannings \& Emerson (1994). The $1.3 \mathrm{~mm}$ flux density for BP Tau was taken from Dutrey, Guilloteau, \& Simon (2003). 3 - 1.3 mm flux densities from Beckwith et al. (1990). $4-1.3 \mathrm{~mm}$ flux densities from Osterloh \& Beckwith (1995). $5-1.3 \mathrm{~mm}$ flux densities from Motte \& André (2001). 6 - Additional submillimeter flux densities (Adams, Emerson, \& Fuller 1990; Beckwith \& Sargent 1991; Mannings 1994) were used in determining $\alpha .7$ - These sources have anomalous continuum slopes, and so are excluded from the analysis in $\S 3.3$. Two of these objects with $850 \mu \mathrm{m}$ detections are known to be variable centimeter radio sources (V773 Tau and V410 Tau). Because V410 Tau has no signatures of disk emission throughout its SED, we assume that its submillimeter flux is not from a dust disk, and instead use a 3- $\sigma$ upper limit of $5 \mathrm{mJy}$ at $850 \mu \mathrm{m}$ in all the analysis. V773 Tau has a slight infrared excess, and so we consider the submillimeter emission to be from the disk. The anomalous slopes for the other sources could be due to contamination from non-disk emission at the longest wavelengths, or errors in the absolute calibrations at different wavelengths. See the Appendix regarding CY Tau. 8 - References for submillimeter maps of some Class I and FS YSOs in the literature. See Young et al. (2003) for maps of 04016+2610, 04166+2706 (also Shirley 
et al. (2000)), 04169+2702, 04239+2436 (also Chini et al. (2001)), 04248+2612, 04295+2251,04302+2247, 04361+2547, and $04381+2540$ (also Hogerheijde \& Sandell (2000)). See Hogerheijde \& Sandell (2000) for maps of 04325+2402 and $04368+2557$ (also Chini et al. (2001)). Chandler \& Richer (2000) provide maps of HL Tau and Sandell \& Weintraub (2001) provide maps of L1551 IRS 5. Maps of the extended submillimeter emission around T Tau are provided by Weintraub et al. (1999). 
Table 2. Results of SED Fits ${ }^{\mathrm{a}}$

\begin{tabular}{|c|c|c|c|c|c|c|c|c|}
\hline $\begin{array}{l}\text { Object } \\
(1)\end{array}$ & $\begin{array}{c}T_{1}[\mathrm{~K}] \\
\quad(2)\end{array}$ & $\begin{array}{c}q \\
(3)\end{array}$ & $\begin{array}{c}M_{d}\left[\mathrm{M}_{\odot}\right] \\
\quad(4)\end{array}$ & $\begin{array}{c}\Delta \\
(5)\end{array}$ & $\begin{array}{l}\tilde{\chi}_{\nu}^{2} \\
(6)\end{array}$ & $\begin{array}{c}\nu \\
(7)\end{array}$ & $\begin{array}{l}T_{c}[\mathrm{~K}] \\
\quad(8)\end{array}$ & $\begin{array}{c}\text { notes } \\
(9)\end{array}$ \\
\hline AA Tau & 129 & 0.56 & $1.3 \pm 0.2 \times 10^{-2}$ & 0.31 & 4.7 & 8 & 15 & $1,2,3$ \\
\hline AB Aur & 367 & 0.45 & $4.4 \pm 0.6 \times 10^{-3}$ & 0.17 & 2.6 & 6 & 65 & 1,2 \\
\hline BP Tau & 117 & 0.64 & $1.8 \pm 0.4 \times 10^{-2}$ & 0.42 & 2.0 & 4 & 11 & 1,2 \\
\hline CI Tau & 152 & 0.56 & $2.8 \pm 0.6 \times 10^{-2}$ & 0.36 & 1.7 & 9 & 15 & $1,2,3$ \\
\hline CW Tau & 204 & 0.62 & $2.4 \pm 0.4 \times 10^{-3}$ & 0.24 & 5.7 & 6 & 27 & 2,3 \\
\hline DE Tau & 130 & 0.55 & $5.2 \pm 0.8 \times 10^{-3}$ & 0.25 & 0.6 & 3 & 19 & 1,2 \\
\hline DF Tau & 126 & 0.74 & $4 \pm 1 \times 10^{-4}$ & 0.28 & 3.6 & 3 & 23 & 1,2 \\
\hline DG Tau & 288 & 0.51 & $2.4 \pm 0.3 \times 10^{-2}$ & 0.29 & 2.7 & 13 & 40 & $1,2,3,4$ \\
\hline DH Tau & 109 & 0.55 & $3.3 \pm 0.7 \times 10^{-3}$ & 0.24 & 2.4 & 4 & 19 & 1,2 \\
\hline DK Tau & 175 & 0.70 & $5 \pm 1 \times 10^{-3}$ & 0.34 & 0.6 & 4 & 18 & 1,2 \\
\hline DL Tau & 149 & 0.62 & $9 \pm 2 \times 10^{-2}$ & 0.58 & 0.6 & 12 & 10 & $2,3,4$ \\
\hline DM Tau & 111 & 0.51 & $2.4 \pm 0.4 \times 10^{-2}$ & 0.34 & 2.0 & 6 & 14 & 2,3 \\
\hline DN Tau & 117 & 0.60 & $2.9 \pm 0.6 \times 10^{-2}$ & 0.43 & 1.5 & 5 & 11 & 2,3 \\
\hline DO Tau & 193 & 0.52 & $7 \pm 1 \times 10^{-3}$ & 0.23 & 2.3 & 12 & 33 & $1,2,3,4$ \\
\hline DQ Tau & 143 & 0.60 & $1.9 \pm 0.5 \times 10^{-2}$ & 0.36 & 0.4 & 4 & 14 & 2,3 \\
\hline DR Tau & 216 & 0.58 & $1.9 \pm 0.3 \times 10^{-2}$ & 0.32 & 1.5 & 10 & 27 & $1,2,3,4$ \\
\hline DS Tau & 97 & 0.67 & $6 \pm 1 \times 10^{-3}$ & 0.39 & 1.0 & 4 & 11 & 1,2 \\
\hline FT Tau & 121 & 0.58 & $1.4 \pm 0.2 \times 10^{-2}$ & 0.34 & 5.0 & 8 & 13 & 2,3 \\
\hline FV Tau & 190 & 0.53 & $1.1 \pm 0.2 \times 10^{-3}$ & 0.17 & 1.4 & 4 & 38 & 2 \\
\hline FX Tau & 118 & 0.63 & $9 \pm 2 \times 10^{-4}$ & 0.24 & 0.6 & 2 & 21 & 1,2 \\
\hline FZ Tau & 148 & 0.73 & $2.0 \pm 0.6 \times 10^{-3}$ & 0.33 & 1.9 & 2 & 17 & 2 \\
\hline GG Tau & 172 & 0.56 & $2.3 \pm 0.8 \times 10^{-1}$ & 0.76 & 1.0 & 8 & 10 & 2,3 \\
\hline GM Aur & 136 & 0.44 & $2.5 \pm 0.5 \times 10^{-2}$ & 0.28 & 2.1 & 6 & 30 & 2,3 \\
\hline GO Tau & 90 & 0.62 & $7 \pm 2 \times 10^{-2}$ & 0.62 & 1.2 & 7 & 7 & 2,3 \\
\hline GV Tau & 339 & 0.46 & $2.8 \pm 0.3 \times 10^{-3}$ & 0.16 & 1.5 & 7 & 78 & 2,3 \\
\hline Haro 6-13 & 181 & 0.47 & $1.1 \pm 0.1 \times 10^{-2}$ & 0.23 & 0.9 & 11 & 33 & $2,3,4$ \\
\hline HK Tau & 140 & 0.44 & $4.5 \pm 0.5 \times 10^{-3}$ & 0.18 & 2.8 & 9 & 28 & $1,2,3,5$ \\
\hline HL Tau & 277 & 0.42 & $6.5 \pm 0.8 \times 10^{-2}$ & 0.36 & 0.7 & 12 & 33 & $1,2,3,5$ \\
\hline HN Tau & 164 & 0.56 & $8 \pm 2 \times 10^{-4}$ & 0.18 & 1.9 & 2 & 33 & 1,2 \\
\hline HP Tau & 201 & 0.47 & $1.0 \pm 0.3 \times 10^{-3}$ & 0.14 & 5.9 & 4 & 160 & 1,2 \\
\hline IP Tau & 107 & 0.63 & $2.8 \pm 0.6 \times 10^{-3}$ & 0.29 & 1.1 & 3 & 15 & 2 \\
\hline IQ Tau & 121 & 0.60 & $2.2 \pm 0.3 \times 10^{-2}$ & 0.40 & 2.4 & 5 & 12 & $1,2,3$ \\
\hline IT Tau & 104 & 0.61 & $1.5 \pm 0.3 \times 10^{-3}$ & 0.25 & 5.1 & 2 & 17 & 1,2 \\
\hline $\mathrm{LkCa} 15$ & 117 & 0.52 & $4.8 \pm 0.9 \times 10^{-2}$ & 0.41 & 2.2 & 5 & 13 & 1,2 \\
\hline RY Tau & 342 & 0.66 & $1.8 \pm 0.3 \times 10^{-2}$ & 0.34 & 1.4 & 13 & 52 & $1,2,3,4$ \\
\hline SU Aur & 264 & 0.48 & $9 \pm 3 \times 10^{-4}$ & 0.14 & 3.2 & 4 & 66 & 1,2 \\
\hline $\mathrm{T}$ Tau & 338 & 0.45 & $8.2 \pm 0.9 \times 10^{-3}$ & 0.19 & 1.9 & 12 & 62 & $1,2,3,5$ \\
\hline UX Tau & 132 & 0.41 & $5.1 \pm 0.7 \times 10^{-3}$ & 0.17 & 3.3 & 5 & 31 & 1,2 \\
\hline UY Aur & 226 & 0.53 & $1.8 \pm 0.3 \times 10^{-3}$ & 0.18 & 1.8 & 5 & 47 & 1,2 \\
\hline UZ Tau & 167 & 0.61 & $6 \pm 1 \times 10^{-2}$ & 0.49 & 2.1 & 9 & 13 & $1,2,3$ \\
\hline V710 Tau & 112 & 0.58 & $1.9 \pm 0.4 \times 10^{-2}$ & 0.37 & 0.6 & 5 & 12 & 2,3 \\
\hline V836 Tau & 97 & 0.58 & $1.0 \pm 0.3 \times 10^{-2}$ & 0.34 & 0.6 & 5 & 12 & 2 \\
\hline V892 Tau & 461 & 0.58 & $9 \pm 1 \times 10^{-3}$ & 0.25 & 1.3 & 8 & 58 & 2,3 \\
\hline V955 Tau & 139 & 0.59 & $5 \pm 1 \times 10^{-4}$ & 0.19 & 6.2 & 2 & 27 & 2 \\
\hline
\end{tabular}

aThe table columns are: (1) - object name; (2) - best-fit value of the temperature at $1 \mathrm{AU}$ in $\mathrm{K}$ (typical errors are \pm a few $\mathrm{K}$ ); (3) - best-fit value of the radial power law index of the temperature profile (typical errors are \pm 0.02 ); (4) - best-fit value of the disk mass for $\beta=1 ;(5)$ - fraction of the $850 \mu \mathrm{m}$ flux density from optically thick regions 
in the disk; (6) - reduced chi-squared statistic; (7) - number of degrees of freedom in the fit (i.e., number of datapoints - number of fitted parameters $[=3]$ ); (8) - inferred characteristic temperature in $\mathrm{K}$ from inversion of Equation 1; (9) - notes on literature sources for the data as follows: $1=10 \mu \mathrm{m}$ photometry from Kenyon \& Hartmann (1995), $2=\operatorname{IRAS}$ photometry $(12,25,60,100 \mu \mathrm{m})$ from Weaver \& Jones $(1992), 3=$ submillimeter photometry from Beckwith \& Sargent (1991), $4=$ submillimeter photometry from Mannings \& Emerson (1994), $5=$ submillimeter photometry from Adams, Emerson, \& Fuller (1990). The largest values of $\tilde{\chi}_{\nu}^{2}$ usually are due to structure in the infrared SED, where the errors on flux densities are low, or slightly inconsistent absolute calibration in the submillimeter. 
Table 3. Multiple Star Systems ${ }^{\mathrm{a}}$

\begin{tabular}{|c|c|c|c|c|c|c|c|}
\hline $\begin{array}{c}\text { Object } \\
(1)\end{array}$ & $\begin{array}{c}\theta_{p}\left[{ }^{\prime \prime}\right] \\
(2)\end{array}$ & $\begin{array}{l}\text { ref }^{b} \\
(3)\end{array}$ & $\begin{array}{c}a_{p}[\mathrm{AU}] \\
(4)\end{array}$ & $\begin{array}{l}\text { Object } \\
\text { (1) }\end{array}$ & $\begin{array}{c}\theta_{p}\left[{ }^{\prime \prime}\right] \\
(2)\end{array}$ & $\begin{array}{l}\mathrm{ref}^{\mathrm{b}} \\
(3)\end{array}$ & $\begin{array}{c}a_{p}[\mathrm{AUU}] \\
(4)\end{array}$ \\
\hline $04113+2758$ & 4.0 & 18 & 560 & HK Tau & 2.4 & 7 & 340 \\
\hline $04325+2402$ & 8.2 & 18 & 1140 & HN Tau & 3.1 & 8 & 430 \\
\hline $04248+2612$ & 4.6 & 18 & 640 & HO Tau & 6.9 & 11 & 970 \\
\hline $\mathrm{CoKu} \mathrm{Tau} / 3$ & 2.1 & 7 & 290 & HP Tau & 0.02 & 10 & 3 \\
\hline CZ Tau & 0.33 & 8 & 46 & HV Tau & $0.03,4.0$ & 6 & 4,560 \\
\hline DD Tau & 0.56 & 8 & 79 & IS Tau & 0.22 & 9 & 31 \\
\hline DF Tau & 0.09 & 16 & 13 & IT Tau & 2.4 & 7 & 340 \\
\hline DK Tau & 2.5 & 6 & 350 & IW Tau & 0.28 & 8 & 39 \\
\hline DQ Tau & $\mathrm{sb}$ & 14 & 0.05 & $\mathrm{~J} 1-4872$ & 3.3 & 16 & 460 \\
\hline DS Tau & 7.1 & 11 & 990 & L1551 IRS 5 & 0.30 & 1 & 42 \\
\hline FF Tau & 0.03 & 10 & 4 & $\mathrm{LkCa} 3$ & $\mathrm{sb}, 0.48$ & 11,16 & $0.03,67$ \\
\hline FO Tau & 0.16 & 8 & 22 & LkCa 7 & 1.0 & 8 & 140 \\
\hline FQ Tau & 0.78 & 8 & 110 & $\mathrm{LkH} \alpha 332 / \mathrm{G} 1$ & 0.22 & 8 & 31 \\
\hline FS Tau & 0.25 & 8 & 35 & $\mathrm{LkH} \alpha 332 / \mathrm{G} 2$ & 0.28 & 8 & 39 \\
\hline FV Tau & 0.72 & 8 & 101 & RW Aur & 1.4 & 7 & 200 \\
\hline FV Tau/c & 0.74 & 6 & 104 & St 34 & $\mathrm{sb}$ & 20 & $\ldots$ \\
\hline FW Tau & $0.16,2.3$ & 12,16 & 22,320 & $\mathrm{~T} \mathrm{Tau}^{\mathrm{c}}$ & $0.10,0.70$ & 17,8 & 14,98 \\
\hline FX Tau & 0.90 & 9 & 130 & UX $\mathrm{Tau}^{\mathrm{c}}$ & $0.14,2.7,5.9$ & $15,7,7$ & $20,380,830$ \\
\hline GG Tau ${ }^{\mathrm{d}}$ & 0.26 & 8 & 36 & UY Aur & 0.88 & 9 & 120 \\
\hline GH Tau & 0.33 & 9 & 46 & $\mathrm{UZ} \mathrm{Tau}^{\mathrm{c}}$ & sb, $0.35,3.7$ & $19,9,7$ & $0.1,49,520$ \\
\hline GK Tau & 2.5 & 7 & 340 & V410 Tau & $0.07,0.29$ & 16 & 10,41 \\
\hline GN Tau ${ }^{\mathrm{d}}$ & 0.04 & 6 & 6 & V710 Tau & 3.2 & 7 & 450 \\
\hline GV Tau & 1.2 & 3 & 170 & V773 Tau & 0.11 & 9 & 15 \\
\hline Haro 6-28 & 0.66 & 8 & 92 & V807 Tau & $0.02,0.37$ & 12,9 & 3,52 \\
\hline Haro 6-37 & 2.7 & 8 & 370 & V826 Tau & $\mathrm{sb}$ & 0 & 0.05 \\
\hline HBC 351 & 0.61 & 8 & 85 & V892 Tau & 4.0 & 13 & 560 \\
\hline HBC $352 / 3$ & 8.6 & 8 & 1200 & V927 Tau & 0.29 & 8 & 41 \\
\hline HBC $354 / 5$ & 6.3 & 11 & 880 & V928 Tau & 0.18 & 8 & 25 \\
\hline HBC $356 / 7$ & 2.0 & 11 & 280 & V955 Tau & 0.33 & 8 & 46 \\
\hline HBC $360 / 1$ & 7.2 & 8 & 1010 & VY Tau & 0.66 & 8 & 92 \\
\hline HBC 412 & 0.70 & 8 & 98 & ZZ Tau & 0.03 & 12 & 4 \\
\hline
\end{tabular}

aThe table columns are: (1) - object name; (2) - projected separation in arcseconds for each pair (sb denotes a spectroscopic binary); (3) - reference for each separation measurement (see b); (4) - projected semimajor axis in $\mathrm{AU}$, assuming a distance of $140 \mathrm{pc}$ (spectroscopic binary separations are the best-fit values of $a \sin i)$.

${ }^{\mathrm{b}}$ Projected separations, $\theta_{p}$, are average values from the following sources: $0=$ Mundt et al. (1983); $1=$ Rodríguez et al. (1986); $2=$ Simon et al. (1987); 3 = Leinert \& Haas (1989); 4 = Haas, Leinert, \& Zinnecker (1990); $5=$ Leinert et al. (1991); $6=$ Simon et al. (1992); $7=$ Reipurth \& Zinnecker (1993); $8=$ Leinert et al. (1993); 9 = Ghez, Neugebauer, \& Matthews (1993); $10=$ Richichi et al. (1994); $11=$ Mathieu (1994); $12=$ Simon et al. (1995); $13=$ Leinert, Richichi, \& Haas (1997); $14=$ Mathieu et al. (1997); 15 = Duchêne et al. (1999); $16=$ White \& Ghez (2001); $17=$ Tamazian (2004); $18=$ Duchêne et al. (2004a); $19=$ Martín et al. (2005); $20=$ White \& Hillenbrand (2005).

${ }^{\mathrm{c}}$ These high-order multiple systems were assigned various flux densities and disk masses for the analysis described in $\S 3.5$ and represented in Figure 11 and Table 4. T Tau - The stellar components are too close to rule out a large circum-triple disk from current interferometric observations, so each separation is assigned the same flux/mass. UX Tau - High-resolution $1.3 \mathrm{~mm}$ observations show that A is the only component 
with a disk (Jensen \& Akeson 2003), so the 2 separations ( $a_{p}=380$ and $\left.830 \mathrm{AU}\right)$ are assigned the same flux/mass value, and the close binary B is given a flux density equal to the completeness limit (10 mJy) and a corresponding disk mass from Equation 5. UZ Tau - We adopt the result of Jensen, Koerner, \& Mathieu (1996) that the $\mathrm{W}$ close binary contributes $\sim 19 \%$ of the total flux density in the system, and scale the flux densities and disk masses accordingly for each separation. HV Tau - We assume that C is the sole component with a disk because the AB close binary has a Class III SED, and C has been shown in the optical to have an edge-on disk (Stapelfeldt et al. 2003). The AB separation ( $a_{p}=4 \mathrm{AU}$ ) is assigned a flux density equal to the completeness limit $(10 \mathrm{mJy})$ and a corresponding disk mass from Equation 5 .

${ }^{\mathrm{d}}$ GG Tau: a quadruple source, these numbers refer only to the A close binary, as the B system falls outside the $850 \mu \mathrm{m}$ SCUBA beam. GN Tau: White \& Ghez (2001) note a significantly larger projected separation, $\theta_{p}=0{ }^{\prime \prime} 33$. 
Table 4. Multiplicity Effects on Disk Properties ${ }^{\mathrm{a}}$

\begin{tabular}{lcrr}
\hline \hline \multicolumn{1}{c}{ samples } & $a_{c}[\mathrm{AU}]$ & $P\left(F_{\nu}\right)$ & \multicolumn{1}{c}{$P\left(M_{d}\right)$} \\
\hline close vs. wide & 50 & $85-91 \%$ & $33-54 \%$ \\
close vs. single & 50 & $9-60 \%$ & $\leq 7 \%$ \\
wide vs. single & 50 & $53-76 \%$ & $53-80 \%$ \\
close vs. wide & 100 & $97-99 \%$ & $\geq 99 \%$ \\
close vs. single & 100 & $26-70 \%$ & $34-83 \%$ \\
wide vs. single & 100 & $77-91 \%$ & $65-89 \%$ \\
\hline Class II, close vs. wide & 50 & $90-94 \%$ & $42-73 \%$ \\
Class II, close vs. single & 50 & $75-90 \%$ & $16-33 \%$ \\
Class II, wide vs. single & 50 & $4-14 \%$ & $89-96 \%$ \\
Class II, close vs. wide & 100 & $94-98 \%$ & $\geq 98 \%$ \\
Class II, close vs. single & 100 & $83-90 \%$ & $91-98 \%$ \\
Class II, wide vs. single & 100 & $12-38 \%$ & $18-38 \%$ \\
\hline
\end{tabular}

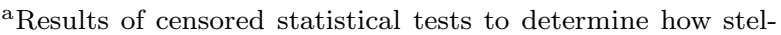
lar companions affect circumstellar disks. See also a graphical comparison in Figure 11. Two-sample tests were performed on the categories listed in the left column and described in the text (§3.5): close binaries (with $a_{p} \leq a_{c}$ ), wide binaries (with $\left.a_{p}>a_{c}\right)$, and single stars. The probabilities that the $850 \mu \mathrm{m}$ flux densities $\left(P\left(F_{\nu}\right)\right)$ or disk masses $\left(P\left(M_{d}\right)\right)$ are drawn from different parent populations are given in the last two columns. The ranges in the probabilities are representative of the various statistical tests. 
Table 5. Summary of Submillimeter Properties ${ }^{\mathrm{a}}$

\begin{tabular}{lcccccccc}
\hline \multicolumn{1}{c}{ sample } & $N_{f}$ & $f_{\text {smm }}$ & $N_{M_{d}}$ & median $M_{d}\left[\mathrm{M}_{\odot}\right]$ & $\sigma\left(M_{d}\right)[\mathrm{dex}]$ & $N_{\alpha}$ & $\operatorname{median} \alpha$ & $\sigma(\alpha)$ \\
\hline Class I & 16 & $1.00 \pm 0.25$ & 16 & $3 \times 10^{-2}$ & 0.59 & 16 & 2.51 & 0.54 \\
Flat-Spectrum & 9 & $1.00 \pm 0.33$ & 9 & $4 \times 10^{-3}$ & 0.53 & 7 & 2.02 & 0.70 \\
Class II & 74 & $0.86 \pm 0.11$ & 64 & $3 \times 10^{-3}$ & 0.67 & 34 & 1.79 & 0.51 \\
Class III & 54 & $0.07 \pm 0.04$ & 4 & $6 \times 10^{-4}$ & 0.26 & 0 & $\ldots$ & $\ldots$ \\
WTTS & 61 & $0.15 \pm 0.05$ & 9 & $2 \times 10^{-3}$ & 0.65 & 5 & 1.73 & 0.27 \\
CTTS & 74 & $0.91 \pm 0.11$ & 67 & $4 \times 10^{-3}$ & 0.72 & 40 & 2.02 & 0.62 \\
multiples & 61 & $0.66 \pm 0.10$ & 40 & $2 \times 10^{-3}$ & 0.74 & 19 & 2.13 & 0.52 \\
singles & 92 & $0.58 \pm 0.08$ & 53 & $1 \times 10^{-2}$ & 0.64 & 38 & 2.01 & 0.60 \\
\hline total & 153 & $0.61 \pm 0.06$ & 93 & $5 \times 10^{-3}$ & 0.71 & 57 & 2.01 & 0.57 \\
\hline
\end{tabular}

a The $N$ values are the total numbers of sources in each subsample. $f_{\mathrm{smm}}$ is the fraction of those objects which were detected at a submillimeter wavelength along with the 1- $\sigma$ Poisson counting error. Median values of disk masses and submillimeter continuum slopes are given, along with the standard deviations $(\sigma)$ of those values. The $\sigma\left(M_{d}\right)$ values are on a $\log$ scale.

Table 6. Outer Disk Evolution: Survival Analysis Tests ${ }^{a}$

\begin{tabular}{lrrr}
\hline \hline \multicolumn{1}{c}{ samples } & \multicolumn{1}{c}{$P\left(F_{\nu}\right)$} & \multicolumn{1}{c}{$P\left(M_{d}\right)$} & \multicolumn{1}{c}{$P(\alpha)$} \\
\hline Class I vs. Flat-Spectrum & $63-85 \%$ & $>99.2 \%$ & $74-87 \%$ \\
Class I vs. Class II & $>99.96 \%$ & $>99.9999 \%$ & $>99.7 \%$ \\
Class I vs. Class III & $>99.9999 \%$ & $>99.9999 \%$ & $\ldots$ \\
Flat-Spectrum vs. Class II & $89-94 \%$ & $60-83 \%$ & $1-36 \%$ \\
Flat-Spectrum vs. Class III & $>99.9999 \%$ & $>99.9999 \%$ & $\ldots$ \\
Class II vs. Class III & $>99.9999 \%$ & $>99.9999 \%$ & $\ldots$ \\
WTTS vs. CTTS & $>99.9999 \%$ & $>99.9999 \%$ & $26-65 \%$ \\
\hline
\end{tabular}

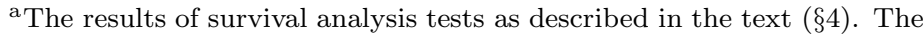
values of $P\left(F_{\nu}\right), P\left(M_{d}\right)$, and $P(\alpha)$ are the probabilities that the $850 \mu \mathrm{m}$ flux densities, disk masses, and submillimeter continuum slopes, respectively, of the two samples in the first column are drawn from different parent populations. The ranges of $P$ values are representative of the various statistical tests. See Figure 13 for a graphical representation of this comparison. 